\title{
And Yet They Move: \\ The Impact of Direction of Deviance on Stereotype Change
}

\author{
Dissertation \\ zur Erlangung des akademischen Grades \\ doctor philosophiae (Dr. phil.)
}

vorgelegt dem Rat der Fakultät für Sozial- und Verhaltenswissenschaften der Friedrich-Schiller-Universität Jena

von Dipl.-Psych. Mirjam Dolderer

geboren am 07.04.1978 in Schwäbisch Gmünd 


\section{Gutachter:}

1. Prof. Dr. Amélie Mummendey, Universität Jena

2. Prof. Dr. Klaus Rothermund, Universität Jena

3. Prof. Dr. Stephan Lessenich, Universität Jena

Tag des Kolloquiums: 22.04.2008 


\section{ACKNOWLEDGEMENTS}

I am grateful to many people who helped, supported and 'distracted' me while I worked on this dissertation. First of all I would like to express my gratitude to my advisor Amélie Mummendey for the trust, encouragement and especially for the stimulating theoretical discussions, which always reminded me of the 'big picture' again. But not just for the scientific training, also for the encouragement while deciding on the professional future, I am very grateful. Likewise, I am equally thankful to Klaus Rothermund for very helpful feedback on the theoretical argument, supportive and fruitful critiques, and additionally the support in methodological questions. I am much obliged to Thorsten Meiser for his very helpful advises while starting this project. Furthermore, I am deeply grateful to Vincent Yzerbyt whom I visited while working on this dissertation. I acquired many scientific skills while staying in Louvain-la-Neuve, Belgium. Thanks to Ilka, Maria, Mauro, Maya, Muriel, Natascha, Nicole, and Sarah for providing comments on chapters of this thesis. I learned a lot about English grammar from you. Thanks to the DFG (German Research Council) for financial support and the great opportunity to travel and meet people from the other sites. I would also like to express my gratitude to the faculty of the IGC who demonstrated an amazing instinct when appointing doctoral students. I experienced the fit among the fellows as 'supercongruent'. It was a pleasure working and living in this environment. Thank you, Nicole, without you 'pre-testing' the IGC, I might have ended up somewhere else. I would like to thank the Jena-friends inside and outside the IGC for your support and distraction and making this place feel like home. Thank you, Marcel, for spending evenings in our favourite place and discussing everything even research. Lastly, I would like to thank my brother and my parents who always have been extremely supportive. 


\section{TABLE OF CONTENTS}

ACKNOWLEDGEMENTS. . .3

TABLE OF CONTENTS .4

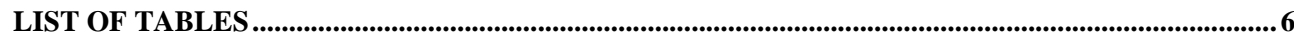

1. Introduction: You've Seen One, You've Seen Them All.......................................................................... 7

2. Stereotypes: Cognitive Templates to Simplify Your Life …….............................................................12

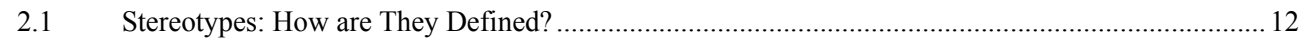

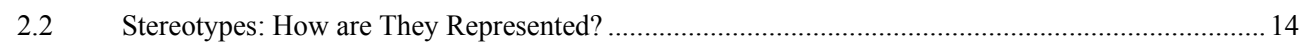

2.3 Stereotypes: Why do They Exist? .......................................................................................... 17

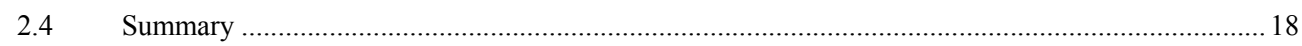

3. Exemplar-Category-Relations I: Supercongruent vs. Incongruent.....................................................19

4. Models of Change.................................................................................................................................................. 22

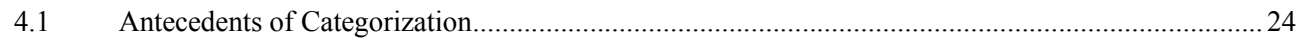

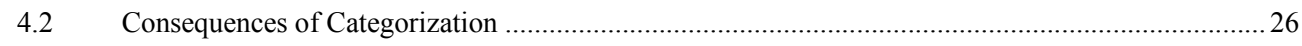

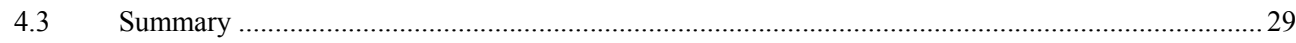

5. Exemplar-Category-Relations II: Who's Typical, Who's Not........................................................................ 31

6. Super- vs. Incongruent: Perceived Typicality and Stereotype Change...................................................... 34

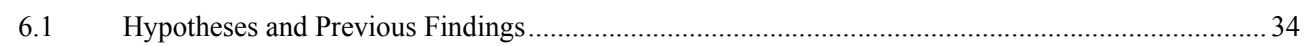

6.2 Overview of the Present Research................................................................................................ 38

7. Part I: Asymmetry in Perception and Impact of Super- and Incongruent Exemplars........................... 40

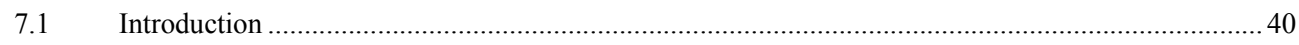

7.2 Study 1: Differences Between Incongruent, Congruent and Supercongruent Exemplars .................. 41

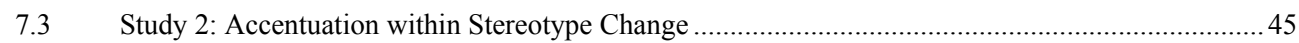


7.4 Study 3: Stereotype Change Following In- and Supercongruent Exemplars....................................... 48

7.5 Study 4: Supercongruent or Incongruent by Means of Different Comparison Categories .................. 54

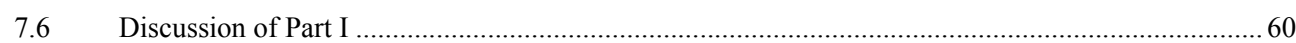

8. Part II: Investigating the Underlying Process (Moderator 1: Mind the Deviance)................................64

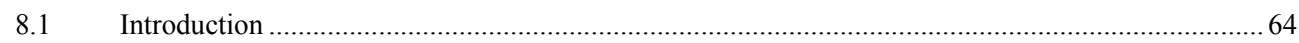

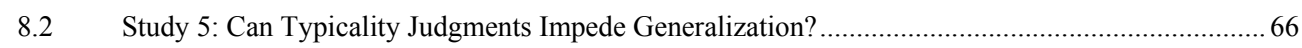

8.3 Study 6: Can Typicality Judgments Draw Focus on Similarities or Dissimilarities? ........................ 76

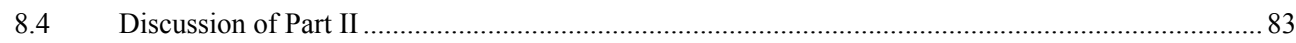

9. Part III: The Positive Side of Subtyping (Moderator 2: Getting to Know the Deviant).........................86

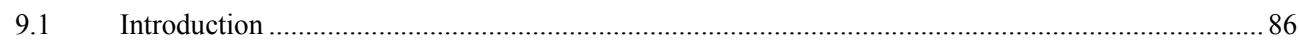

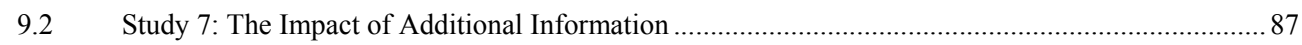

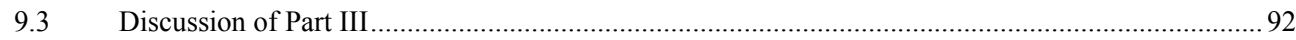

10. General Discussion...................................................................................................................................97

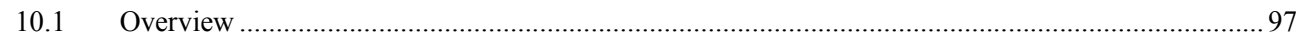

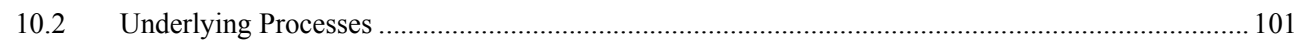

10.3 Results in Light of Previous Findings and Theories................................................................. 107

10.4 Limitations and Suggestions for Future Research ....................................................................... 111

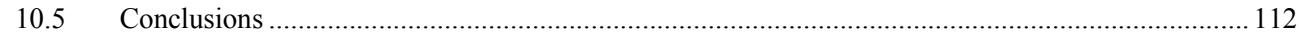

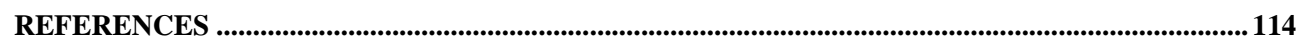

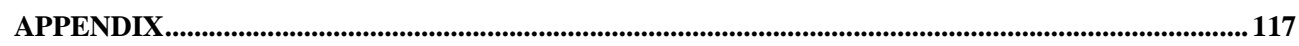

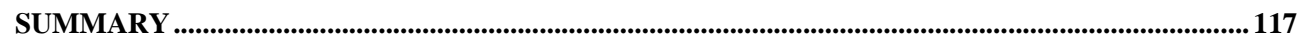

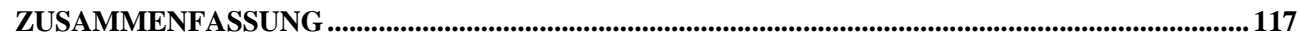

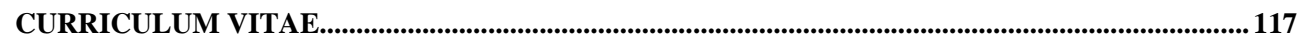

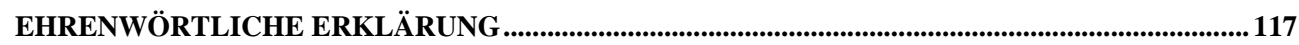




\section{LIST OF TABLES}

Table 1: Means and standard deviations on perceived deviance and perceived typicality of incongruent, congruent and supercongruent exemplars (Study 1) ...........................................44

Table 2. Means and standard deviations on difference score (IQ-estimations for both groups) and trait judgment for the target category after confrontation with either an incongruent, congruent, or supercongruent exemplar (Study 2)

Table 3. Means and standard deviations on perceived typicality and stereotype judgment after confrontation with either an incongruent or supercongruent exemplar (Study 3)........53

Table 4. Means and standard deviations on perceived typicality and assimilation of the stereotype after confrontation with either an incongruent or supercongruent exemplar (Study 4).

Table 5. Means and standard deviations on exemplar judgment, perceived typicality, central tendency and perceived homogeneity dependent on direction of deviance (incongruent, congruent, or supercongruent) and order of measurement (Study 5)

Table 6. Means and standard deviations on stereotype judgment dependent on kind of exemplar (congruent or supercongruent) and focus of comparison (similarity, dissimilarity, default) (Study 6).

Table 7. Means and standard deviations on expectancy values dependent on experimental condition (no exemplar, exemplar without additional information, exemplar plus additional information) (Study 7)

Table 8. Means and standard deviations on warmth ratings for different categories dependent on experimental condition (exemplar without additional information, exemplar plus additional information, no exemplar) (Study 7) 


\title{
1. Introduction: You've Seen One, You've Seen Them All...
}

\author{
"A man is taking a walk in Central Park in New York. Suddenly he sees \\ a little girl being attacked by a pit bull dog. He runs over and starts \\ fighting with the dog. He succeeds in killing the dog and saving the \\ girl's life. A policeman who was watching the scene walks over and \\ says:" You are a hero, tomorrow you can read it in all the newspapers: \\ "Brave New Yorker saves the life of little girl" The man says: - "But I \\ am not a New Yorker!" The policeman answers, "Oh, then it will say in \\ the newspapers in the morning: 'Brave American saves life of little \\ girl’" "But I am not an American!" - says the man. "Oh, what are you \\ then?", asks the cop. The man says: - "I am a Saudi!" The next day the \\ newspapers says: "Islamic extremist kills innocent American dog."
} (Joke cited on www.islamictorrents.net)

Right after the controversial Dutch filmmaker Theo van Gogh was killed by a Moroccan immigrant in 2004, surveys conducted in the Netherlands reflected a marked deterioration of the image of Muslims in public opinion. A sign, put up at the site of crime, stated "Stop islamization of the Netherlands". When asked about Muslims living in the Netherlands, forty per cent of polled Dutch people said that Muslims should not feel at home in the Netherlands anymore. The reactions were not just limited to verbal expressions: A couple of mosques and Koran schools have been burned down in the aftermath of the murder. Evidently, a country otherwise known for its liberal attitudes towards immigration was shaken by an eruption of xenophobia. These specific events seem to be symptomatic for a trend that can be noticed in many Western European countries. People's stereotypic views about Muslims as being fanatic and violent seem to polarize with every single terrorist that makes it into the media. Comparably, the stereotype about immigrants as being prone to crime, is boosted by each crime report which mentions national affiliation. 
The German Press Council states in the German Press Code (2006) that "there must be no discrimination against a person because of his/her sex, a disability or his/her membership of an ethnic, religious, social or national group." This code has important implications for reports on crimes as further noted: "When reporting crimes, it is not permissible to refer to the suspect's religious, ethnic or other minority membership unless this information can be justified as being relevant to the readers' understanding of the incident. In particular, it must be borne in mind that such references could stir up prejudices against minorities." Despite this explicit code, many news reports do mention the group membership of a perpetrator or suspect. Jäger and colleagues (Jäger, Cleve, Ruth, \& Jäger, 1998) have analyzed 1565 articles in the German media and counted that about half of them included specifications about suspects' national affiliation. The researchers just screened those reports that appeared in one of the large German newspapers or political magazines. Practice might differ even more from the guidelines of the press council if taking into account those news that are published on internet by non-professionals.

The following news report, written by an user of the online magazine "Shortnews", exemplifies this practice, and the report's discussion in an online bulletin board demonstrates how people might deal with such an information. The hobby-journalist states: "Two teenagers have been arrested in Munich for attempted murder of a taxi driver. A 16-year old Turkish and a 17-year-old German have attacked their victim in order to get money for drugs." [...] "40-times the young Turkish stabbed the taxi driver." (Shortnews, 14.01.07; translated from German). Another user commented on this news in an online bulletin board ("Klamm-Forum”) the following way: „Who wonders ....again a Turkish involved... (I feel sorry for the normal Turkish, who also get this bad reputation falsely)." This user, on the one hand, regards the category Turkish as a good explanation for the deed, on the other hand, he is able to acknowledge that many Turkish might not be like this. This apparently contradictory reaction seems to be common as other comments reveal.

A less dramatic example of a group member who might reflect badly on the image of her group was found in another online bulletin board ("PC-Freunde"). Some users were engaged in the discussion whether Paris Hilton lacks intelligence. This American celebrity proofed her profound geographical knowledge in a court case by locating London outside of Britain and stating that everybody in Europe speaks French. This news was commented 
by several users with "typical blond" because it seems to fit the cultural stereotype of dumb blonde. A female user, who took part in the discussion group and who was obviously known by the other users to be blond as well, was immediately described as the exception that proves the rule. Is that what typically happens: Extreme, but yet stereotypic exemplars are regarded as representative for the group as a whole, whereas non-stereotypic exemplars are taken as an exception?

The present dissertation can be characterized as phenomenon driven, since the basic research question developed upon the tragic events taking place in the Netherlands in 2004. In this case, obviously people have generalized from the behaviour of one extremist to a whole cultural group. Based on previous social psychological research, it was difficult to tell whether this reaction should be regarded as exceptional, comprehendible just by the outstanding emotionality of the incidents and by previous Islamist terrorist attacks, or whether stereotypes in general are susceptible to polarization. Hence, it was not my first aim to test a certain theoretical model, but to better understand the incidents of these developments. Nevertheless, it deemed necessary to translate the basic question into theoretical terms in order to develop assumptions based on previous research.

Research dealing with stereotype change has focused on how stereotypic views of other groups can be altered by confronting observers with members of the group who do not confirm the stereotype (Rothbart \& John, 1985). However, deviance from the stereotype has been seen as more valuable in one direction than in another. Because the ultimate goal is to improve inter-group relations, researchers' interest has focused on how moderately positive group members would modify an otherwise problematic image of the group (incongruent relation). Unfortunately, as the above examples suggest, it does happen that people encounter deviant individuals who are in fact more negative than the average group member. What van Gogh's murder, the Turkish teenager, and Paris Hilton have in common is that their behaviours are deviating from what is expected, but still they are not opposed to the prevalent stereotypes. Their deviance from the stereotype is just a matter of degree, namely more extreme than expected. So far, researchers have mainly differentiated between congruent and incongruent exemplars (cf. chapter 3). The kind of exemplar this dissertation is dealing with will be called supercongruent hereinafter. I would like to stress that being incongruent or supercongruent is not just a matter of valence. You might think, for exam- 
ple, of a Harvard professor winning the Nobel price: We would certainly expect all members of the category "Harvard Professor" to be highly intelligent, but would we deem them all worthy of receiving this distinction? We have to acknowledge that there is more to our stereotypes than incongruent and congruent exemplars.

We have little knowledge so far about the circumstances under which supercongruent members of a group lead to a polarization of the stereotype and under which circumstances the stereotype remains unaffected because the exemplar is recognized as being quite extreme. In light of these extreme members, other category members might even appear quite moderate, and hence the stereotype might be attenuated. Stereotype change research remains largely silent regarding the influence of such supercongruent members. The present dissertation addresses the question whether exemplars who are more extreme than our expectations (supercongruent) have a different impact on stereotypes than exemplars that are deviating in the other direction (incongruent).

The term change will be used in a broad sense within this dissertation: It comprises gradual change such as attenuation or polarization, as well as complete extinction or alteration of a certain stereotype. Whether an individual has the power to change a stereotype largely depends upon a categorization decision, which means whether the exemplar is seen as a legitimate or illegitimate member of the group. Previous research has addressed the question when deviating group members are included or excluded from judgments about the group as a whole. Based on these findings, it might be possible to propose assumptions about how the direction of deviance might affect the decision whether to include or exclude an exemplar. By learning about the impact of supercongruent exemplars on stereotypes, it might be possible to interrupt a process of stereotype polarization.

The second chapter summarizes definitional issues about stereotypes, the third one outlines some assumptions about the nature of supercongruent and incongruent exemplars, and the fourth chapter resumes relevant theoretical models and previous research dealing with changing inter-group attitudes. The contact hypothesis, various models of stereotype change, the inclusion/exclusion model, and the accentuation theory, are outlined to answer the following questions: 1) what determines whether an exemplar will exert influence on the representation of a category, and 2) what are the possible kinds of influence. The fifth chapter treats the question of when an exemplar is perceived as typical for a group and 
mainly summarizes assumptions of the self-categorization theory. The sixth, and final theoretical chapter, points to several implications of direction of deviance based on the previous research on stereotype change. Two basic hypotheses about the perception and impact of supercongruent exemplars will be established which were tested in the first four studies described in chapter seven. Chapter eight and chapter nine deal with moderating variables to the basic effect, which means they are devoted to the question whether certain circumstances exist in which the basic effect identified in chapter seven does not occur. Learning about these circumstances should help to understand the underlying processes involved in the basic effect (chapter 8), and to develop possible attempts for intervention (chapter 9).

I would like to stress again that a reader should bear in mind that the present dissertation is not aiming at testing a certain theoretical model but at understanding a certain phenomenon which has not yet been the focus of research. In order to devise predictions, diverse lines of research will be presented in the theoretical chapter. To a certain extent, these lines of research can be contradicting and most of these contradictions will remain unresolved. 


\title{
2. Stereotypes: Cognitive Templates to Simplify Your Life
}

\author{
"For the most part, we do not first see, and then define, we define \\ first and then see." \\ Lippmann (1922; p. 81)
}

Muslims, teenagers, blondes, housewives, homosexuals - each label will trigger certain associations and expectations about the particular traits of group members. Social scientists as well as lay people refer to these expectations by the term 'stereotype'. Targets of stereotyping might be social groups, whose membership is based on age, nationality, religious affiliation, taste of music, profession etc. However, not just social groups, also categories of objects can be the target of preformed expectations called stereotypes. We might expect wholefood products to be rather expensive and branded products to possess high quality. Although the usage of the term 'stereotype' seems to be self-explicatory, there are some questions that need to be addressed in order to clarify what is meant by 'stereotype' within this dissertation. The question of how stereotypes can be defined, how they are represented, and why they exist, will be briefly discussed because these issues might determine whether and under which conditions stereotypes are likely to change (Hilton \& von Hippel, 1996). Obviously, a detailed discussion of these questions is beyond the scope of the present theoretical introduction (for a more detailed overview see Hamilton \& Sherman, 1994; Hilton \& von Hippel, 1996; Schneider, 2004).

\subsection{Stereotypes: How are They Defined?}

The term ,stereotype' is rooted in the Greek words ,stereos' and ,typos'. ,Stereos' can be translated with ,rigid, constant, invariable'; ,typos' means ,pattern, type, imprint, trace, impression'. The journalist Walter Lippmann (1922) was the first within the social sciences to use the term in its present meaning. He employed the term stereotype to name "general cognitive structures that people tend to have because the real environment is altogether too big, too complex, and too fleeting for direct acquaintance." [... "We have to reconstruct it on a simpler model before we can manage it" (Lippmann, 1922, p. 16). Hence, 
he assumed that people tend to use preformed pictures about the social environment in order to simplify social perception. Lippmann regarded stereotypes as efficient in dealing with everyday life, but also pointed to the pitfall of ending up with a biased and erroneous picture of the world.

Gordon W. Allport (1954) further elaborated the concept of stereotypes in his book "The Nature of Prejudice". Allport defined the term stereotype as "an exaggerated belief associated with a category" (p. 187). This definition comprises two aspects: First, stereotypes are category-based beliefs. We do not employ the term stereotype to refer to the belief that John Cleese ${ }^{1}$ is funny. But we do use the term if someone holds the belief that all British are humorous. Second, stereotypes are exaggerated or false beliefs. Although current definitions mostly do not include the notion of accuracy or exaggeration anymore, many of Allport's predictions have held up over the last fifty years.

In the last four decades, stereotypes have been defined in many different ways within social psychology. Generally spoken, stereotypes are often regarded as the cognitive dimension of inter-group attitudes (Ashmore \& Del Boca, 1981), while prejudice is conceptualized as the affective dimension. Definitions of stereotypes basically differ in regard to the assumed accuracy, to the question whether they are individual beliefs or whether they have to be culturally shared to qualify as a stereotype, to the question of valence, and level of comparison, meaning whether the attribute value is typically compared with that of other groups or with that of other attributes related to the same group. For example, stereotypes have been defined as "those generalizations about a class of people that distinguish that class from others" (McCauley, Stitt, \& Segal, 1980, p. 197; see also Campbell, 1967; Ford $\&$ Stangor, 1992). This definition emphasizes the aspect of differentiation as central to the conception of stereotypes and thereby renders stereotypes rather context dependent. Since this dissertation deals with the question whether the social context exerts influence on stereotypes, this definition is deemed important. However, it already carries heavy theoretical baggage. Presumably, not all stereotypes are to the same extent interrelated with other categories (Goldstone, 1996). Therefore, I would like to adopt a broader definition of stereotypes, one which constitutes the standard viewpoint within social cognition. Ashmore and Del Boca have defined stereotypes as "a set of beliefs about the personal attributes of a

\footnotetext{
${ }^{1}$ British comedian who is known for being one of the founding members of Monty Python.
} 
group of people" (1981, p. 16). This definition stays silent about all of the above mentioned issues. It shares with Allport's definition the scope on beliefs, meaning cognitive structures, and not affective reactions, but it does not narrow down the focus on false or exaggerated beliefs. Furthermore, it allows to incorporate negative as well as positive attributes. The target of the beliefs are social groups.

\subsection{Stereotypes: How are They Represented?}

One way of understanding stereotypes is to examine the nature of the cognitive structures on which they are based. As mentioned before, stereotypes are category-based beliefs. The question of how stereotypes are represented in memory corresponds to the question of how categories are represented. Roughly speaking, categories can be understood as the outcome of a partitioning of our surrounding, for example, in social psychologists and clinical psychologists, or in social scientists and natural scientists. Typically, we do not treat every object as a single entity. The machine, for example, on which this dissertation is written can be described by brand and serial number, but it can also be referred to as a computer, and everyone will have some idea in mind, for example, that this machine is useful for data handling. Hence, category labels seem to be stored in memory together with some information about the features of category members.

To date, four popular major social cognitive approaches exist which aim at clarifying how categories are stored in memory: prototype models, exemplar-based models, associative networks, and connectionist models (for a detailed overview see Fiske \& Taylor, 1991; Smith, 1998; Smith \& Queller, 2004). Most of the research dealing with stereotype change is either related to prototype - or exemplar-based models of stereotypes. ${ }^{2}$ Therefore, the gist of these two classes of models will be briefly covered below, while associative networks and connectionist models will not be elaborated on.

Prototype models assume that knowledge of social categories is stored in memory in form of prototypes, which are conceptualized as abstract representations of the typical features (Posner \& Keele, 1968; 1970; Reed, 1972). Within the research on natural object categories, a prototype was defined by Rosch $(1975$, p. 193) as the "clearest case" or "best example" of a category, so, any real or hypothetical instance that is perceived the most typi-

\footnotetext{
${ }^{2}$ For an exception see Queller \& Smith (2002). Their approach to stereotype change is based on a connectionist model.
} 
cal. More specifically, prototypes were assumed to comprise average feature values (Medin, 1989; Smith \& Medin, 1981). This implies that a mean or modal value of each feature is associated with the category. According to prototype-models, categorization, which means the decision to regard a certain stimuli as belonging to a certain category, is based on the similarity between an exemplar and the prototypes of alternative categories (Medin, 1989). The similarity between an exemplar and the prototype of a certain category is also called, prototypicality, or goodness-of-fit of the exemplar.

Furthermore, prototype-models assume that social categories are organized hierarchically (e.g., Brewer, Dull, \& Lui, 1981; Johnston \& Hewstone, 1992; Maurer, Park, \& Rothbart, 1995), existing of basic-level categories, subtypes, and subgroups. The term $b a-$ sic-level category describes the most commonly utilized classification (e.g., based on race, gender, or age). On a hierarchically lower level, subtypes were defined as comprising those people who disconfirm the stereotype, or to put it differently, who are the exceptions to the overall stereotype. Subgroups were conceptualized as being made up of people who manifest the stereotype in a different way than people in other subgroups (see Medin, Lynch, \& Solomon, 2000, Richards \& Hewstone, 2001). The category "the elderly", for example, might consist of such diverse subgroups, as "the grandmotherly type", or "elder statesman". As we will see later, these assumptions carry important implications for theorizing about stereotype change.

Pure prototype-models rest on the assumption that one fixed prototype is stored in memory. The prototype should be the same no matter in which context a judgment is made, or to put it differently: This model does not allow for any context-sensitivity. However, if you try to think of the prototypical CDU politician, you might end up with a different picture dependent on whether you compare them with politicians of "DIE LINKE" or "NPD". Quite some empirical evidence exists for the context sensitivity of prototypes (e.g., Corneille \& Judd, 1999; Goldstone, 1996; Haslam et al., 1995; cf. chapter 5), which causes major problems for pure prototype models.

Exemplar-based models stress the role of concrete instances (rather than general, abstract properties) in the mental representation of categories and actual experiences with category members are the substrate of the mental representation (Linville, Fischer, \& Salovey, 1989; Medin \& Schaffer, 1978; Smith \& Zárate, 1992). New information is classi- 
fied according to its similarity with stored exemplars. General information, such as the typical properties of a category, is computed at the time a judgment is needed by retrieving the representation of multiple individual exemplars from memory ${ }^{3}$. Which exemplars will be included in the computation depends on their accessibility.

Most of the exemplar-based models and the prototype models basically differ on the issue when prototype abstraction occurs: either at the time of initial encounters with members of the category (prototype-based) or at the time when information is retrieved from memory in order to come up with a judgment (exemplar-based) (e.g., Linville \& Fischer, 1993). Nowadays, pure prototype- or exemplar-based models are not very popular. Most researchers within social psychology allude to one of the hybrid versions of the two, which assume that a stereotype representation contains a mix of both, exemplars along with abstract knowledge (Judd \& Park, 1988; Park \& Hastie, 1987; Smith \& Medin, 1981; Smith \& Zárate, 1990). Some researchers argued that prototype-based representations might be strongest for those categories, with which people have little experience, but yet strong expectancies. These expectancies could be, for example, based on cultural socialization (Smith \& Zárate, 1990). Exemplar-based representations, on the contrary, were suggested to dominate when beliefs about a social category are not yet very developed and when single exemplars are highly accessible within memory (see Higgins, 1996).

So far, it has been stated that categories are either represented by single exemplars, by average feature values (prototype), or that they contain both. During the last two decades, however, several researchers from both traditions claimed that representations do also comprise perceived variability (Hamburger, 1994; Linville et al., 1989; Judd, Ryan, \& Park, 1991). Perceived variability can be defined as "the extent to which category members are viewed as being widely dispersed about the mean of the attribute (Linville et al., 1989, p. 165).

Clearly, all these models lead to different conclusions about the rigidity or sensitivity of stereotypes to change and about the specific aspects of a stereotype that are likely to change (Hilton \& von Hippel, 1996). According to pure prototype models, stereotypes are insulated from change by the formation of subtypes leading to a rather rigid view of stereo-

\footnotetext{
${ }^{3}$ Not all exemplar-based models do allow for the computation of general information (e.g., Nosofsky, 1986). But because these models have difficulties to account for the formation of group stereotypes, they will not be considered here.
} 
types (e.g., Johnston \& Hewstone, 1992, cf. chapter 4.1). According to exemplar-based models, stereotypes should be rather context sensitive as the judgment is created on the spot and thereby influenced by salient exemplars (e.g., Schwarz \& Bless, 1992, cf. chapter 4.2). A rather heterogeneous group of researchers (some allude to prototype models, others to exemplar models) assumes that the variability components of stereotypes do change, whereas other aspects remain stable (e.g., Garcia-Marques \& Mackie, 1999; Hamburger, 1994; Paolini, Hewstone, Rubin, \& Pay, 2004). The topic of stereotype change will be covered in more detail in chapter 4.

\subsection{Stereotypes: Why do They Exist?}

Why do people hold and utilize stereotypes? Although it is not the scope of this dissertation to discuss in detail the different functions of stereotypes, the general pattern that emerges from this literature is worth noting, especially as it relates to stereotype change (for an overview see Snyder \& Miene, 1994). What Lippman (1922) has already assumed by describing stereotypes as efficient has been demonstrated more than seventy years later within social cognition research. Macrae and colleagues have shown in a dual-task paradigm that stereotypes help to save cognitive capacity (Macrae, Milne, \& Bodenhausen; 1994), and Bodenhausen has demonstrated that we use stereotypes especially when we are tired and lack cognitive resources (Bodenhausen, 1990). Stereotypes seem to serve as quick and easy heuristics (Bodenhausen \& Wyer, 1985). Besides making information processing easier, further purposes were suggested to be served by stereotypes, for example justification of the status quo (Jost \& Banaji, 1994). This utility account of stereotypes has two important implications. Firstly, stereotypes should be based on attributes that differentiate groups from each other in order to fulfil their simplifying function or to justify social hierarchies (see Ford \& Stangor, 1992; McCauley \& Stitt, 1978). The second implication is more directly related to the topic of change: Given the important functions stereotypes seem to serve, they should be valuable to people and they might be reluctant to give them up even when facing contradictory evidence. 


\subsection{Summary}

Stereotypes were defined as a set of beliefs about the personal characteristics of a group of people. Some researchers pointed to the differentiating nature of stereotypes, which means that a stereotype is likely to consist of such attributes that distinguish the target category from other categories. Stereotypes were argued to be represented either in an abstract fashion by prototypes, or consist of concrete instances, such as exemplars, or comprise both. Representations of social categories were furthermore argued to include variability information. As a matter of fact, pure exemplar- or prototype-based models are becoming more and more uncommon, but still they have influenced diverse theories about stereotype change. It has been demonstrated that stereotypes fulfil important functions, such as simplification or justification, which should render them rather valuable. As stated before, these basic issues regarding stereotypes might determine when and how stereotypes change. A more detailed discussion of factors influencing stereotype change will follow within chapter 4. But first I would like to depict diverse relations between exemplar and category in order to introduce more solidly the concept of supercongruent exemplars. 


\section{Exemplar-Category-Relations I: Supercongruent vs. Incon- gruent}

Shirin Ebadi, awarded with the Nobel Peace Prize in 2003, and Mohammed Atta, one of the five hijackers to crash the first plane into the World Trade Center in 2001, are both members of the category 'Muslims', but obviously, both relate quite differently to the stereotype of this category.

One of the central factors characterizing the relationship between an exemplar and a category is the congruency of the exemplar with the stereotypical expectations. Is the exemplar in line with our expectations for the group or does it deviate? Accordingly, researchers have typically differentiated between a congruent and an incongruent relationship. Yet, there is some ambiguity about the meaning of incongruency. Some researchers have conceptualized incongruency as the opposite of the stereotype (e.g., O'Sullivan \& Durso, 1984), others have described it as being typical for another, contrasting category (e.g., Brewer et al., 1981). Brewer and colleagues, for example, used items that were descriptive of the category "young people" (e.g., has a lot of nervous energy) as incongruent stimuli for the category "elderly". 4

As mentioned before, stereotypes are supposed to consist of average feature values (Medin, 1989; Smith \& Medin, 1981), which are either stored in the form of a prototype or computed at the time of judgment by averaging the accessible exemplars. People should have an idea about the extent to that members of a category possess a certain trait. This assumption has important implications for the relationship between categories and exemplars because it allows for the possibility of another kind of deviance besides incongruency: Exemplars could deviate in the direction of the stereotype by being even more extreme than one would have expected.

Returning to the above example: If we focus on the stereotype of Muslims as being fundamentalist and fanatic, then Shirin Ebadi certainly is not in line with the category. She is what has been called incongruent to the category's stereotype. Mohammed Atta, although

\footnotetext{
${ }^{4}$ In many but probably not in all circumstances it will be the same attributes that are opposite of the stereotype and typical for another contrasting group. One example of a stereotypic attribute for the contrasting category "young people" in Brewer and colleagues' study (1981) was "fusses over her appearance". Yet, the opposite of this attribute was not part of the stereotype of "the elderly" as described within that study.
} 
most people probably would attribute fundamentalism and fanaticism to him, is such an extreme exemplar that his fit with the stereotype becomes questionable as well. The present dissertation addresses the impact of this second type of exemplars, which I will refer to as supercongruent.

Supercongruent and incongruent exemplars differ in several important ways. Firstly, even if you imagine a supercongruent and an incongruent exemplar sharing exactly the same amount of objective deviance from a category's prototype ${ }^{5}$, the deviance from supercongruent exemplars to the prototype should be perceived more as a quantitative deviance, while the deviance from an incongruent exemplar to the prototype is quite likely to be perceived as qualitative in nature. Supercongruent exemplar and prototype share the same attribute, they just differ in extremity. Incongruent exemplars, on the other hand, most probably do not possess the attribute in question. For example, if we hold the belief that members of a certain group are rather intelligent, then supercongruent exemplars would probably be described as highly intelligent (possess the same attribute), whereas incongruent exemplars either might not be describable by the attribute intelligent because it is not characteristic for them, or they might be even described as unintelligent, simple-minded, or ignorant (do not possess the same attribute).

Two further differences between these two kinds of exemplars are based on the notion that stereotypes are differentiating in nature. As mentioned before, several theorists included in their definition of stereotypes a differentiation between a certain category from other categories (e.g., McCauley et al., 1980, Campbell, 1967; Ford \& Stangor, 1992). Accordingly, theorists have described incongruency as being typical of another group (e.g., Brewer et al., 1981). Given that supercongruent exemplars were conceptualized as those exemplars that deviate in direction of the stereotype, they can be understood as deviating away from the prototype in the opposite direction as relevant comparison categories. This leads to one of the central distinctions between the two kinds of deviance: Incongruent exemplars, if they are taken as representative members of the category, decrease the distinctiveness between two groups, whereas supercongruent ones increase it.

\footnotetext{
${ }^{5}$ The usage of the term prototype within this dissertation does not necessarily imply that the prototype is stored in memory, it could be also estimated at the time of judgment by averaging the accessible exemplars.
} 
Included in the notion of differentiation is the fact that for an attribute to qualify as stereotypic for a certain category, the category has to be somehow pronounced on that attribute in comparison with some other categories (e.g., McCauley \& Stitt, 1978). Because a supercongruent exemplar is deviating in the direction of the stereotype, whereas an incongruent exemplar differs in direction of some comparison category, they might differ in extremity. Supercongruent exemplars differ more from the grand mean of all available or salient stimuli, while incongruent exemplars with the same amount of deviance from the prototype are more likely to be rather pale.

To make the point, incongruent and supercongruent exemplars with the same amount of objective deviance from the prototype might differ in respect to 1) the perceived character of the deviance (quantitative vs. qualitative), 2) the direction of deviance in relation to comparison categories (away from comparison category or in direction of comparison category), and 3) their extremity. Based on these differences, several assumptions will be made in chapter 6 , concerning the impact of supercongruent exemplars on a group's stereotype. First, however, I will summarize different models which address stereotype change, such as the contact hypothesis, models of stereotype change, the inclusion/exclusion model, and the accentuation theory. 


\section{Models of Change}

What impact does an encounter with a single group member have on the general belief we hold about the group? While we know much already about the impact of incongruent exemplars, knowledge is rather scarce in respect to supercongruent exemplars. In order to derive assumptions about the impact of supercongruent exemplars, it deems necessary to take a closer look at previous research dealing with stereotype change. In the present chapter, I will outline various models that address the following questions: 1) what are the antecedents determining whether an exemplar will or will not exert influence on the overall judgment, 2) what are the potential kinds of influence, and 3) given that exemplars do influence the overall judgment, what determines the size of this influence? Before going into detail, the contact hypothesis will briefly be summarized, as it is the first and most prominent theory for answering the question of whether a single group member may impact the image of a social category.

After World War II, researchers within the social sciences began to stress the role of contact in prejudice reduction (e.g., Allport, 1954; Newcomb, 1947). The idea is based on the assumption that prejudice largely stems from ignorance. Accordingly, a rather problematic image of a group can be altered by bringing people into contact with single members of the category to overcome this ignorance. This "encounter" does not have to be direct. Rothbart and John (1985) have mentioned as an alternative source of belief change indirect "atmospheric" effects, which could be due to media portrayals for example. Hence, firsthand contact or reports, which indicate that our attitudes towards a certain category are biased, were assumed to convince us to correct our attitudes.

Results to the question of whether a contact experience has the potential to improve inter-group relations are highly diverging. Tropp and Pettigrew (2005) have argued and demonstrated that this inconsistency is largely due to the fact that researchers have focused on different outcomes which bear different patterns of relationship with contact (for similar results see Wolsko, Park, Judd, \& Bachelor, 2003). Those researchers who concentrated on affective dimensions of inter-group attitudes, namely prejudice, have painted a more positive picture of the susceptibility to change, while researchers focusing on the cognitive dimension have been more sceptical (see Rothbart, 1996). Because the focus of this disserta- 
tion lies on the cognitive aspects of inter-group attitudes (stereotypes), I will turn now to research dealing with contact effects on this cognitive aspects.

The rather optimistic view emanating from contact theory was damped by findings of stereotype change research. Rothbart and John (1985) proposed a two-step model in their cognitive analysis of the effects of inter-group contact: First, the inter-group contact must provide clear evidence that disconfirms the stereotype, and second, this experience must become associated with the category. The first precondition is facilitated through personalized contact, as the stereotype will be salient and guide the interpretation of the person's behavior during group-based contact. Ambiguous behavior will be interpreted in light of the stereotype (Kunda \& Sherman-Williams, 1993). However, for the second step to take place, the person has to be categorized as a group member. Hence, categorization lies at the heart of this process. Categorization can be defined as "the process of understanding what some thing is by knowing what other things it is equivalent to and what other things it is different from" (McGarty, 1999, p. 1). Rothbart and John (1985) have pointed to the fact that category membership is not fixed, but variable. Many factors influence where the group boundaries are drawn. According to this model, a trade-off between individualized and group-based contact somehow has to be established.

Several theories, which can be summarized as consistency theories (see Abelson, 1983), draw similar predictions about the various possibilities of how people deal with inconsistent cognitions. We can understand the encounter with an incongruent, or more generally, with a deviating exemplar of a social category as a contradiction within our cognitive system. This state of mind is assumed to be experienced as unpleasant and people should strive to resolve the inconsistency by reorganizing their cognitions (Festinger, 1957; Heider, 1946). An example: Most people in our society hold the stereotype of professors as being intelligent. Imagine, meeting a certain professor, Professor X, who happens to behave in a rather unintelligent manner. This experience should constitute a contradiction within the cognitive system.

Basically, we have three possibilities how to deal with information that contradicts our beliefs: 1) We can make the information fit our beliefs, for example, by carrying out suitable attributions (e.g., it is due to lack of motivation that Professor $\mathrm{X}$ behaves in an unintelligent manner), or 2) we can regard the information and the belief as two distinct in- 
stances which have very little in common by excluding the exemplar from the category (e.g., Professor X is not a "real" professor since he is professor in a conservatory), or 3) we make the belief fit the information by a process of belief change (e.g., professors are not that intelligent $)^{6}$. The following paragraphs will be especially concerned with the last two possibilities (for the role of attributions in stereotype maintenance see e.g., Hewstone, 1990).

All models addressing stereotype change in one way or another deal with the question of categorization: When is an inconsistent exemplar categorized as a member of the stereotyped group? And what is the impact of the categorization process for the judgment about the group and the exemplar? To date, four major classes of models have been proposed, which address the question of how stereotypic views are revised: the bookkeeping model, the conversion model, the subtyping model, and exemplar-based models (see Hilton $\&$ von Hippel, 1996). While the first three models especially emphasize the antecedents of categorization, the last class of models focuses more on the outcomes of categorization.

\subsection{Antecedents of Categorization}

According to the bookkeeping model, stereotypes change incrementally. Every piece of disconfirming information leads to a gradual, minor modification of the stereotype, which was referred to as "fine-tuning" (Rothbart, 1981). The conversion model assumes that stereotypes change in an all-or-non fashion (Rothbart, 1981). Incongruent information does not affect the stereotype until a certain threshold is reached. After crossing the threshold, a critical reevaluation of the stereotype will take place leading to radical change. Minor disconfirming exemplars have no effect. The subtyping model, which is clearly based on a prototype representation of social categories, states that the impact of disconfirming information depends on the structure of the information: Highly incongruent exemplars are very likely to be viewed as an untypical exception and will be set aside. Hence, the overall stereotype is not affected. Moderately incongruent information, on the other hand, is assumed to have some minor impact on the stereotype (Brewer et al., 1981; Weber \& Crocker, 1983).

\footnotetext{
${ }^{6}$ These are the major possibilities. However, there are also others, for example stereotype maintenance through compensation (Seta, Seta, \& McElroy, 2003).
} 
In order to test the three models researchers have varied the extremity of the exemplar information and either concentrated the disconfirming information on a few exemplars or dispersed it over several members so that every exemplar is just moderately deviant (e.g., Weber \& Crocker, 1983). Most of the studies have shown support for the subtyping model of stereotypes, which means that stereotypes change less in the concentrated than in the dispersed condition (e.g., Johnston \& Hewstone, 1992; Johnston, Hewstone, Pendry, \& Frankish, 1994; Kunda \& Oleson, 1995; Weber \& Crocker, 1983). As a matter of fact, disconfirming exemplars commonly do not affect the stereotype and the process of mentally fencing-off the disconfirming exemplar has been called subtyping. Subtyping was defined "as the process by that exemplars who disconfirm, or are at odds with, the group stereotype are mentally clustered together and essentially set aside as exceptions that prove the rule" (Maurer et al., 1995, p. 812). Subtyping can be understood as a change in the cognitive representation of the category, by which it becomes more differentiated.

Departing from this result, and based on the notion that most outgroup stereotypes are rather negative, researcher were subsequently interested in finding ways of how people can be prevented from subtyping disconfirming information and how change can be facilitated instead (e.g., Hewstone, Hassebrauck, Wirth, \& Wänke, 2000; Kunda \& Oleson, 1995, 1997; Richards \& Hewstone, 2001; Yzerbyt, Coull, \& Rocher, 1999). This focus developed since researchers concentrated on stereotype incongruent information.

Goodness-of-fit (perceived typicality) between the exemplar and the category has proven to be one of the major criteria determining whether the attributes of an exemplar will modify the category attributes or not. Perceivers who consider the disconfirming outgroup member as an atypical exemplar change their stereotype less than those who perceive the deviant member to be a typical exemplar of the group (e.g., Johnston \& Hewstone, 1992; Kunda \& Oleson, 1997; Rothbart \& John, 1985; Rothbart \& Lewis, 1988; Wilder, 1984). Weber and Crocker (1983), for example, paired disconfirming information about lawyers (e.g., wearing ill-fitting clothes) either with typical instances of the category (e.g., described as rich, white, married), or with atypical instances (e.g., black, single, poor). Subjects' beliefs were more likely to be affected when the disconfirming exemplars possessed other attributes that rendered them typical. Goodness-of-fit between an exemplar and a category has been manipulated in diverse ways: 1) by varying the amount of stereotypic 
dimensions on which the exemplar is disconfirming (e.g., Johnston \& Hewstone, 1992; Weber \& Crocker, 1983; Wilder, 1984), 2) by varying the extremity of deviance (e.g., Kunda \& Oleson, 1997; Wyer, Sadler, \& Judd, 2002), or 3) by context, namely task instructions (e.g., Bless \& Wänke, 2000).

To make the point, various paradigms all led to the same conclusion of perceived typicality as a main determinant of the impact of one exemplar on the judgment about the category ${ }^{7}$. The same conclusion was drawn by researchers within the inclusion/exclusion model, although they called it "feature-overlap" (Bless, Schwarz, Bodenhausen, \& Thiel, 2001; Schwarz \& Bless, 1992). Authors of the inclusion/exclusion model were arguing for the assignment of the exemplar to the category (inclusion) as the default reaction, unless the exemplar is not highly atypical (Schwarz \& Bless, 1992). Because exclusion was assumed to require extra processing steps, assimilation was argued to be especially likely to occur, whenever processing motivation and /or cognitive capacity are low. This parallels the finding of stereotype change research that subtyping processes, which can be considered as similar to exclusion, demand cognitive capacity (Yzerbyt et al., 1999). Because the inclusion/exclusion model focuses more on the consequences of categorization, it will be outlined more thoroughly within the next chapter.

People seem to justify subtyping or exclusion of an exemplar from the category representation generally by relying on a humble fit between exemplar and category. ${ }^{8}$ Consequently, one could ask what determines the fit between an exemplar and the category. Chapter 5 will summarize the basic arguments to this question.

\subsection{Consequences of Categorization}

Within this chapter, possible outcomes of the exemplar's categorization will be addressed. I will thereby concentrate on the effects on central tendency judgments of a category. However, a growing number of researchers suggests that it is further necessary to consider perceived variability as an outcome variable (e.g., Garcia-Marques \& Mackie, 1999; Hamburger, 1994; cf. chapter 8).

\footnotetext{
${ }^{7}$ Some researchers treated perceived typicality as an outcome of subtyping because they demonstrated that the link between perceived typicality and stereotype change is bidirectional (e.g., Hewstone \& Hamberger, 2000).

${ }^{8}$ Other factors were demonstrated as well, as for example the availability of additional, neutral information to justify subtyping (Kunda \& Oleson, 1995; cf. chapter 9).
} 
The inclusion/exclusion model, which has been briefly mentioned in the previous chapter, concentrates on the outcomes of categorization for the target category as well as for the exemplar (Schwarz \& Bless 1992; 2007). While stereotype change researchers mostly manipulate the features of an exemplar and test whether this influences the decision to generalize or to subtype (e.g., Kunda \& Oleson, 1997), researchers testing the inclusion/exclusion model usually manipulate categorization more directly while keeping information about the exemplar and the category constant (e.g. Bless et al., 2001; Bless \& Wänke, 2000). Furthermore, the inclusion/exclusion model is more general compared to classic stereotype change models in the sense that it does not distinguish between cognitive aspects of social judgments (stereotypes) or more affect-laden judgments. Further, it assumes the same processes for social, as well as non-social categories.

The inclusion/exclusion model is based on an exemplar-representation and rests on the notion that stereotypic judgments are assumed to be formed on the spot (Smith \& Zárate, 1992; cf. chapter 2.2). Therefore, judgments should be influenced by salient stimuli within the context. Models referring to an exemplar-based representation allow for greater change than the previously mentioned models. Usually, researchers within this tradition use the term 'context dependency' instead of change. The term used implies that the influence might not be durable in time. Whether long-term belief change or short-term context sensitivity, the influence might take two different directions, namely assimilation or contrast.

\subsubsection{Consequences of Categorization: Direction of Influence}

Schwarz and Bless $(1992 ; 2007)$ have argued that judgments require a mental representation of the judgmental target and a representation of the standard against which the target is judged. Both representations were assumed to include chronically as well as temporarily accessible information. Thereby, judgments should be influenced by the present context. The contextual information can be used in two ways: Either as part of the representation of the judgmental target, leading to assimilation, or as a standard of comparison, leading to contrast. This decision is based on a categorization process (cf. chapter 4.1).

Assimilation was defined as a positive relation between the contextual stimuli (e.g., exemplar) and the judgment (Schwarz \& Bless, 2007). Contrast was defined as a negative relation between the two. The inclusion of a certain exemplar into the representation of a category was assumed to trigger assimilation processes in the judgment of the category and 
the exemplar. Hence, contact-based stereotype change was assumed to consist of a tradeoff: For example, the image of a category that is stereotypically seen as rather negative, could improve by means of a positive exemplar. At the same time, the view on the exemplar might suffer from being assimilated towards the categories' prototype (e.g., Bless et al., 2001). The same trade-off was demonstrated to be involved in contrast effects.

"Subtyping" and "exclusion", as already mentioned, can be understood as similar processes. However, as Richards \& Hewstone (2001) have noted "there has been considerable debate concerning the consequences of subtyping" (p. 56). While exclusion was argued to always lead to contrast effects, those researchers referring to subtyping and who have included a control condition either demonstrated contrast effects as a consequence of subtyping (e.g., Kunda \& Oleson, 1997, Study 4), or reduced assimilation (e.g., Yzerbyt et al., 1999, Study 2), or observed no change at all (e.g., Yzerbyt et al., 1999, Study 1).

\subsubsection{Consequences of Categorization: Size of Influence}

Assimilation, as well as contrast effects, were argued to be more pronounced the more extreme the salient stimuli are (Schwarz \& Bless, 1992). For example, given that an exemplar is included in the representation of a certain category, its effect on the central tendency judgment of the category will be larger when the exemplar is extremely deviant from the other information included in the representation. When considering the key-points of the previous chapter, a caveat becomes apparent here: Extremely deviating exemplars are likely to exert more influence, but at the same time, they are more likely to be subtyped or excluded because of perceived atypicality than less extremely deviating exemplars.

Another determinant of the size of assimilation and contrast effects is the amount of additional information included in the representation of the category or the representation of a comparison standard. A given piece of information will exert less influence the more other information is accessible (Bless, Igou, Schwarz, \& Wänke, 2000). However, the overall size of assimilation or contrast effects will be bigger the more contextual information is added (Schwarz \& Bless, 2007). This principle was referred to as the set-size effect.

Another theory that has been applied to category formation and change, is the accentuation theory (e.g., Krueger, 1991; 1992; Krueger \& Rothbart, 1990; Krueger, Rothbart, \& Sriram, 1989; Tajfel, 1969). In contrast to most of the previously mentioned research, researchers interested in the accentuation theory typicality taught their partici- 
pants to differentiate between two categories and then confronted them with a huge list of exemplars, that deviated from the just formed expectations in one or the other direction. Researchers aimed at demonstrating that not all exemplars exert the same size of influence. Exemplars that enhance differences between categories exert a bigger impact in terms of assimilation than exemplars that reduce the difference. Krueger and colleagues were building on the work by Tajfel and Wilkes (1963), who demonstrated that exaggeration of intercategory differences is a general perceptual phenomenon. Krueger and colleagues (1989) were the first to test this accentuation effect with respect to ongoing changes in judgments due to new information. In a first phase, participants in their studies learned to distinguish between two distributions of three-digit-numbers which were presented in different fonts. Subjects learned that one distribution was made up of higher (or lower) values than the other (comparison) distribution. Repeatedly, participants had to rate the cumulative means of both categories. During the learning phase the ratings were quite accurate. In the second half of these experiments, a list of new numbers was presented that either enhanced or reduced inter-category differences. Perceived mean change was bigger when differences were enhanced rather than reduced. Krueger and colleagues (1989) attributed this effect of accentuated mean change to a biased averaging process. More specifically, this asymmetry was argued to be based on memory processes (e.g., Krueger, 1992; Krueger \& Rothbart, 1990). Krueger and Rothbart (1990, Exp. 3) could demonstrate that difference-enhancing stimuli were remembered better than difference-attenuating stimuli. The data, however, did not allow to draw a conclusion about the nature of this memory effect. Did the expectations that developed in the first phase of the experiment influence the memory? Were differenceenhancing stimuli recalled better because of their greater extremity and thereby salience? Or, were difference-attenuating stimuli erroneously recalled as contextual stimuli? These questions remain open.

\subsection{Summary}

Whenever people are confronted with information about single exemplars that somehow deviates from their expectations for the category, they try to resolve this inconsistency by either assimilating their perception to the stereotype, by attributing their behaviour to some external sources, or by understating the typicality of the exemplar for the category. 
If these resolutions seem implausible, they might finally change their stereotype. Whereas prejudice, the affective facet of inter-group attitudes, seems more susceptible to change, stereotypes have been proven to be rather rigid. They are protected against disconfirmation by allowing for a hierarchical structure consisting of subtypes that comprise the disconfirming exemplars. Perceived typicality of the exemplar has been conceived as the major antecedent for subtyping. The inclusion/exclusion model has also pointed to the important role of typicality (or feature-overlap) as a cause for inclusion or exclusion. While researchers agreed on the important role of perceived typicality in stereotype change, less agreement exists concerning the outcomes of subtyping (or exclusion, respectively). Based on the inclusion/exclusion model, an exemplar that has been excluded from the representation of the category should lead to a contrast effect. Researchers studying stereotype change either found no impact of a subtyped exemplar on the category, or they provided evidence for attenuated assimilation, or for a contrast effect. Researchers dealing with the accentuation effect have stressed the fact that not all exemplars affect the overall judgment equally. More weight is given to exemplars that enhance, rather than reduce, inter-category differences. Accentuation theory was one of the precursors of a theory that explained categorization as a more dynamic process and which will be outlined in the following chapter, namely self-categorization theory. Because perceived typicality has proven to be such a critical factor in stereotype change, I will turn now to the question of what impacts perceived typicality. 


\section{Exemplar-Category-Relations II: Who's Typical, Who's Not}

It has been argued in the previous chapter that perceived typicality or goodness-offit is one of the main determinants of whether an exemplar will exert influence on the stereotype of a category or not. In the present chapter, it will be outlined what determines perceived typicality.

So far, it has been mentioned in various passages that exemplars differ in perceived typicality. Although this notion seems plausible, it has not always been taken for granted. Within the classical view of categories, which draws back to Aristotle, it has been assumed that categories consist of defining features. Every exemplar that possesses these features is an equally good member of the category. The severest problem for the classical view is that it seems impossible to state this defining features. ${ }^{9}$ As a consequence, categories became thought of as "fuzzy-sets" (McCloskey \& Glucksberg, 1978).

In the 1970s, a series of studies on natural language concepts demonstrated that the structure within a category is graded, implying that some members of a category are better examples than others (e.g., Rosch \& Mervis, 1975). This graded structure within a category was described as the typicality gradient. Sparrows, for example, seem to be perceived as more typical for the category 'birds' than penguins. As mentioned before, a prototype was defined by Rosch (1975) as the clearest case or best example of a category within a certain context. So, any real or hypothetical instance that is perceived the most typical.

But how does the internal structure of a category arise? Or, how does the prototype form? Rosch and Mervis (1975) proposed an argument based on family resemblance. According to these authors, members of a category become viewed as prototypical, the more their attributes overlap with other members of their category and the less they overlap with attributes of members of other categories. Hence, an intra- and inter-group comparison ought to be involved. Although the work by Rosch is oftentimes cited as an example of a prototype-based model, her notion of typicality as being dependent upon an inter-group comparison clearly contradicts the context-insensitivity of prototypes according to other prototype-models (cf. chapter 2).

According to prototype- and exemplar-based models, categorization decisions are based on similarity judgements. But stimuli can be similar in infinitely many different ways

\footnotetext{
${ }^{9}$ An often cited example is the category "game", for which it is impossible to formulate the defining features.
} 
(Medin, Goldstone, \& Gentner, 1993). A perceiver has to have some idea about the relevant dimensions for the comparison. Therefore, some researchers pointed to the necessity to include prior knowledge and context information into our conception of categorization (Murphy \& Medin, 1985; Smith \& Zárate, 1992). Especially complex categories, such as social categories, became more and more conceptualized as being constructed from working memory instead of being static, stored entities (Eysenck \& Keane, 2000). Researchers within the social identity approach developed a similar stance on categorization, as will be described within the following paragraphs.

Within self-categorization theory (SCT; Turner, Hogg, Oakes, Reicher, \& Wetherell, 1987) categorization is regarded as a dynamic, context-dependent process, rather than an activation of a cognitive structure (Oakes, Haslam, \& Reynolds, 1999). With the aim of developing a theory that explains social categorization and its role in social perception and identification, Turner and colleagues applied and developed the idea imposed by Rosch and Mervis (1975) for social categories. Within SCT all person perception is regarded as the outcome of some categorization process, which can vary in the degree of inclusiveness. Interpersonal and inter-group perceptions differ in their abstractness. Which kind of abstraction and thereby category is applied is, on the one hand, dependent on attributes of the perceiver, such as aims and prior knowledge (Medin et al., 1993). On the other hand, upon the salience of a certain comparative context. Within any given frame of reference categorization is dependent on relative similarities: A set of stimuli is more likely to be categorized as an entity the more the stimuli are perceived to be similar to each other and the more they are perceived to differ from other stimuli in a certain context. This has been called the meta-contrast principle (Turner, 1987).

The meta-contrast ratio can be also used to define the prototypicality of an instance within a certain category (Turner \& Oakes, 1989). Respectively, the prototypicality of an instance also depends on this relative similarity and thereby on an intra- and inter-group comparison. Thus, the exemplar being the most prototypical in a certain context is the one that is the most similar to other members of the category, and most dissimilar to members of other categories (being salient in a certain situation). An example taken from Oakes and colleagues illustrates this principle: “ $[\ldots]$ In a communist party, members wishing to be perceived as prototypical communists must differ in politics from members of more con- 
servative, capitalist parties, but they must not be so ultra-left that they begin to differ significantly from other communists" (Oakes, Haslam, \& Turner, 1998, p. 80). Since the typicality judgment is dependent upon an inter-group comparison, it can just be made on-thespot within a certain context.

Wyer and colleagues (2002) have demonstrated the context sensitivity of the prototype in two studies addressing stereotype change. Participants learned about a target category, which was described as politically liberal and academically motivated, either in the context of another group, which was described as politically conservative, or a group that was described as academically unmotivated. The prototype was expected to be more extreme (polarized) on the dimension which was discriminative in the specific context. Participants later encountered additional members of the target category, who were contradicting the stereotype on both dimensions (political orientation and academic motivation). As a consequence of the polarized prototypes, extremity of deviance was assumed to be bigger on the respective, context relevant attribute dimension. Participants changed their stereotypes more on the dimension that did not differentiate between the two social groups. Disconfirming exemplars were assumed to be perceived as more atypical on the contextrelevant dimension, and this greater atypicality in turn rendered them more likely to be subtyped. The results confirmed, on the one hand, the context sensitivity of the prototype and thereby the categorization theory, and on the other hand, they provided evidence for the pivotal role of perceived typicality in stereotype change.

In sum, the aforementioned argumentation carries two important implications. First, perceived typicality seems to follow the meta-contrast ratio: An exemplar is perceived as more typical, the more it is similar to other members of the category, and the less it is similar to non-members. Second, because typicality depends upon an inter-group comparison, the perception of typicality is context-dependent. The notion of a prototype only makes sense within a certain frame of reference. 


\section{Super- vs. Incongruent: Perceived Typicality and Stereotype}

\section{Change}

\subsection{Hypotheses and Previous Findings}

Various theories about the nature of categories and the process of categorization have been outlined as well as specific models about stereotype change. Previous research has demonstrated that stereotypes can be quite resistant to disconfirming information (cf. chapter 4). Incongruent exemplars, that is, exemplars that are contradicting the stereotype, are often subtyped because of their perceived atypicality. Although other models, such as the inclusion/exclusion model, regard assimilation as the default process, they also stress the fact that atypical exemplars are likely to be excluded from the representation of the category. Especially those researchers who were interested in the impact of one single deviating exemplar, argued and demonstrated that this exemplar will not influence the central tendency aspect of the stereotype (e.g., Hamburger, 1994; Paolini et al., 2004). This result might have been influenced by the decision to concentrate on incongruent exemplars instead of on supercongruent ones.

The basic question of this dissertation is: What is the impact of one supercongruent exemplar on the central tendency facet of a stereotype compared with an incongruent exemplar? Since perceived typicality has been argued and demonstrated before as being a main predictor of exemplar-based change (cf. chapter 4), a second and related question is whether supercongruent and incongruent exemplars are perceived as equally typical.

Research on the representation of natural object categories (Rosch, 1975), as well research based on the social identity approach (SCT; Turner et al., 1987), assumed and demonstrated that perceived typicality is dependent on an intra- and inter-group comparison (cf. chapter 5). The more an exemplar is similar to other members of a group and the more it differs from non-members, the higher will be the perceived typicality (Turner \& Oakes, 1989). As incongruent exemplars have been defined before as deviating in the direction of a comparison category and supercongruent exemplars were now defined as deviating away from a salient comparison category (cf. chapter 3 ), perceived typicality should differ according to the direction of deviance. 
Thus, the first hypothesis tested in this dissertation concerns the difference in perceived typicality: It is predicted that supercongruent exemplars should be perceived as more prototypical than incongruent exemplars, even if they share the same amount of objective deviance from the prototype. Hence, the same objective deviance translates to different degrees of subjective deviance.

Similar reasoning has been tested in the subjective group dynamics model (e.g., Abrams, Hogg, \& Marques, 2005; Abrams, Marques, Bown, \& Henson, 2000). Researchers within this field showed that members who deviate in their opinion towards an opposing group, called "anti-norm deviants", are perceived as more atypical than those who deviate away from the opposing group, called "pro-norm deviants". The reason for that asymmetry in prototypicality was seen in the greater contrast anti-norm deviants pose to the normative direction of their group compared to pro-norm deviants. Also other researchers related to the social identity approach provided evidence that typicality perception is dependent upon an intergroup comparison. Haslam and his colleagues showed that extreme members of an outgroup are perceived as more representative than more moderate members (Haslam et al., 1995). In their study, the same exemplar was either described as being moderate (associated with smaller inter-category differences) or extreme (associated with greater inter-category differences). When participants had to indicate their own attitude before making the typicality judgment, they judged the extreme outgroup exemplar as more typical than the moderate one. This asymmetry has so far been tested in the context of an ingroup-outgroup distinction. However, it seems reasonable to assume that the same principles should also apply without a persons' ingroup being salient as a reference point.

Typicality has proven to be the critical factor for generalization in stereotype change research (cf. chapter 4). Perceivers who consider the disconfirming outgroup member as an atypical exemplar change their stereotype less than those who perceive the deviant member to be a typical exemplar of the group (e.g., Johnston \& Hewstone, 1992; Kunda \& Oleson, 1997; Rothbart \& John, 1985; Rothbart \& Lewis, 1988; Wilder, 1984). Based on these findings and the assumption of differences in perceived typicality, I hypothesized that supercongruent exemplars have a different impact on the group stereotype than incongruent exemplars. 
It is predicted that supercongruent exemplars are likely to be included into the representation of a category and thereby leading to an assimilation of the prototype to the supercongruent exemplar. The impact of incongruent exemplars on the prototype should be smaller because they are more likely to be subtyped due to their lower perceived typciality. Therefore, prototypes should more easily get polarized than attenuated. The classical finding of stereotype change research, namely that deviating exemplars are very likely to be subtyped, should not hold for supercongruent exemplars.

This hypothesis is supported by research on the accentuation theory. As mentioned before, one major difference between incongruent and supercongruent exemplars is that the former ones decrease inter-group distinctiveness while the latter ones increase it (cf. chapter 3). Exaggeration of inter-category differences seems to be a general perceptual phenomenon (Krueger \& Rothbart, 1990; Krueger et al., 1989; Tajfel \& Wilkes, 1963; cf. chapter 4). Because people seem to be prone to maximize inter-category distinctions, assimilation of the prototype to supercongruent information should be very likely to occur. The aim of the present research is to demonstrate that the accentuation principle also applies to existing social stereotypes and single deviating exemplars.

Empirical evidence regarding supercongruent exemplars is scarce so far. Two studies within research on stereotype change, although mainly interested in the effect of incongruent exemplars, comprised a condition that was considered as overly stereotypic. Kunda and Oleson (1995; Exp. 4) included an overly stereotypic condition in order to demonstrate that subtyping due to additional, neutral information is not just a matter of deviance, but of true inconsistency. Participants in their study read an interview with a gay man who either described himself as having stable relationships (incongruent), or as changing partners quite frequently (overly stereotypic). The additional information that the gay man works as an accountant blocked generalization in case of the incongruent exemplar, but not in case of the overly stereotypic exemplar. The chosen research paradigm, however, did not allow for a comparison between the objective deviance of the incongruent and supercongruent exemplar from the prototype. It is not even clear whether the exemplar was actually supercongruent or congruent.

Garcia-Marques and Mackie (1999; Exp. 2), who were interested in the effect of incongruent exemplars on perceptions of variability, included a supercongruent condition in 
one of their studies in order to show that variability judgments are affected by incongruent exemplars and not by any kind of deviating exemplar. By using numerical information (IQvalues), they aimed at making the degree of deviance between incongruent and supercongruent exemplars comparable. Their main interest was in the variability judgment and no adequate measure of stereotype change was included in the study. Participants had to come up with IQ values for 30 randomly sampled members of the group. The deviating exemplar was introduced by providing participants with a first IQ score. The averaged IQ estimations in this study were influenced by the supercongruent as well as the incongruent first score. Effects on this measure, however, could be explained better in terms of anchor effects than in terms of belief change. Therefore, it seems to be important to have a closer look at the effect of supercongruent exemplars on the central tendency of a stereotype.

Research on the accentuation effect also gave rise to the present hypotheses (Krueger, 1991; Krueger \& Rothbart, 1990; Krueger et al., 1989). These studies started off with a stereotype learning phase in which two categories (target and comparison category) were presented by rendering a long list of category members accessible. Some studies dealt with categories made up of three-digit-numbers (Krueger, 1991; Krueger et al., 1989), later, researchers aimed at extending the previous findings on the accentuation effect with traitdescriptive adjectives as stimuli (Krueger \& Rothbart, 1990). Participants learned about a focal category, made up of traits that were neutral in favourability, and either a negative contextual category or a positive contextual category. During the first phase of the experiment categories were not overlapping. Subjects had to rate the favourability of each trait and to estimate both group means at the end of phase one. Phase two, in which participants were presented with another set of 48 traits, was designed to test the idea that differenceenhancing stimuli will influence the overall estimation more than difference-attenuating stimuli. Participants received an additional list of traits for the focal category that either increased favourability or decreased it. For the contextual category, mean favourability of the additional traits was identical to mean favourability during phase 1. Krueger and Rothbart found support for contrast, as well as accentuation effects during phase 2. Contrast effects could be demonstrated by averaging the favourability judgments of the single traits and comparing this index between the two context conditions. Accentuation effects were shown by comparing the average estimated mean for the focal group with the computed average of 
the individual ratings for each context condition. Krueger and Rothbart (1990; Exp. 3) included a surprise recall task at the end of their study to test the idea whether memory processes could be responsible for the accentuation effect. Indeed, memory was worse for the difference-attenuating traits than for the difference-enhancing traits. Krueger and Rothbart were not very specific why exactly they assumed memory to be better for difference enhancing stimuli. As mentioned before, the data did not allow to draw a conclusion about the nature of this memory effect. It could have occurred due to expectancies influencing memory, or due to greater salience of difference-enhancing stimuli based on their greater extremity, or to confusion of difference-attenuating stimuli. Krueger and colleagues did not mention (or test) differences in perceived typicality. Effects might have occurred due to higher perceived typicality of difference-enhancing stimuli based on meta-contrast principles. The question remains whether accentuation is a purely memory-driven phenomenon. As mentioned before, Krueger and colleagues always presented their subjects with a long list of stimuli. In those situations, memory processes might be more important than in cases in which just one exemplar is presented. However, in real life, supercongruent exemplars might be rather rare due to their extremity. Hence, it is quite unlikely that one encounters several supercongruent exemplars at once. Unfortunately, no evidence exists so far about the impact of one single supercongruent exemplar on the prototype of an existing social category.

\subsection{Overview of the Present Research}

The present studies investigated the impact of supercongruent exemplars. In contrast to earlier studies, which addressed similar questions, I a) tested whether supercongruent exemplars can be differentiated from congruent and incongruent exemplars, b) examined the impact of one single supercongruent exemplar on pre-existing stereotypes as well as on artificial stereotypes, c) compared the impact of one supercongruent exemplar to that of one incongruent exemplar with the same objective amount of deviance from the prototype, and d) assessed typicality and mean level change within the same studies. Part II and Part III are summarizing studies that additionally examined moderating factors which e) shed some further light on the underlying processes of the basic effects, and f) provide hints how stereotypes can be prevented from getting polarized. 
Within the first experimental chapter four studies will be presented that address the two main hypotheses concerned with perceived typicality and mean level change (a-d). Various kinds of paradigms were used within the present research to capture belief change and accentuation. Basically, it was tested whether supercongruent exemplars are perceived as more typical than incongruent exemplars and whether they exert a bigger impact on the central tendency of a stereotype than incongruent exemplars. By testing typicality and mean-level change within the same studies, I explored whether these two judgments are causally linked, meaning whether higher perceived typicality of supercongruent exemplars compared with incongruent exemplars is responsible for the higher impact of supercongruent exemplars on the stereotype.

To further clarify the underlying mechanisms, studies presented in Part II investigated whether a generalization of supercongruent information is inevitable. In particular, I was interested whether a polarization of the stereotype due to supercongruent information still occurs when participants' attention is drawn towards the deviance of the exemplar. This idea rests on the assumption that a supercongruent deviance might be easily overlooked by perceivers because the deviance is quantitative in nature. Hence, the default reaction when confronted with a supercongruent exemplar should be an assimilation process, which could be blocked by forcing participants to focus on dissimilarities between exemplar and prototype.

In Part III an interventional hypothesis was tested within a new experimental paradigm (cf. chapter 9). I explored whether the same factors that have been demonstrated to trigger subtyping of incongruent exemplars could possibly block generalization of supercongruent exemplars as well. 


\section{Part I: Asymmetry in Perception and Impact of Super- and Incongruent Exemplars}

\subsection{Introduction}

Within the first empirical part of this dissertation I would like to summarize results concerning the two basic questions, namely 1) whether supercongruent and incongruent exemplars with the same objective amount of deviance from the prototype differ in perceived typicality, and 2) whether their impact on the central tendency of a stereotype differs. Additionally, it was tested whether these processes are causally linked. Based on the meta-contrast principle, I argued that supercongruent exemplars should be perceived as more typical than incongruent exemplars because supercongruent exemplars differ more from members of contrasting categories than incongruent exemplars. Based on this higher perceived typicality and the accentuation effect, supercongruent exemplars should furthermore exert a bigger impact on the overall central tendency judgment of a category than incongruent exemplars. This means that supercongruent exemplars should lead to a stronger assimilation of the prototype than incongruent exemplars.

Study 1 was designed to test whether supercongruent exemplars can be distinguished from congruent exemplars and whether they are perceived as more typical than incongruent exemplars. The study dealt with IQ values of members of a group stereotypically seen as rather intelligent. The numerical nature of IQ values provided the opportunity to create equal distances from the prototype, which was assessed in a pre-test. Study 2 tested whether supercongruent information indeed leads to an accentuation of inter-group differences, whereas incongruent information has no impact. Hence, it addresses the question whether supercongruent exemplars are used by social observers to maximize differences between categories. The study also dealt with IQ values of the same category as Study 1 . Study 3 was carried out to test whether supercongruent exemplars cause stronger assimilation than incongruent exemplars within a paradigm that is more common for stereotype change research. The target category was one which is stereotypically seen as lacking warmth. Study 4 was designed to exclude the alternative hypothesis that this effect is driven 
by the greater extremity of supercongruent compared with incongruent exemplars. For this study, a stereotype learning paradigm about artificial groups was applied.

In order to increase generalizability the stereotypes employed within this set of studies vary in valence and are related to the warmth and competence dimensions of the stereotype-content-model (Fiske, Xu, Cuddy \& Glick, 1999). Fiske and colleagues have argued for two separate and basic dimensions of stereotypes, namely warmth and competence, which can describe most of the stereotypes predominant in most cultures.

I would like to briefly note some technical details which are common to all four studies summarized in the first empirical chapter. In all four studies I aimed at constructing a supercongruent and an incongruent exemplar with an equal amount of deviance from the prototype. Numerical information was used in all studies (test-points in competence and social competence related tests). Based on the finding that prototypes are context dependent (Oakes et al., 1998) a comparison category with an opposing stereotype was rendered salient in most of the studies in order to hold the standard of comparison for all participants constant. Because the present studies aimed at comparing the impact of incongruent and supercongruent exemplars by creating equal distances from the prototype, it was deemed necessary to fix the prototype as far as possible by at least holding the frame of reference constant over pre-test and main study.

\subsection{Study 1: Differences Between Incongruent, Congruent and Super- congruent Exemplars}

\section{$\underline{7.2 .1 \quad \text { Introduction }}$}

The main goal of the first study was to demonstrate that supercongruent exemplars can actually be distinguished from congruent exemplars in the sense that people perceive supercongruent exemplars as deviating from the expectations. Additionally, Study 1 was designed to test the first hypothesis concerned with typicality. Participants should judge supercongruent exemplars as more typical than incongruent exemplars even if they share the same amount of deviance from the prototype. Hence, the same objective deviance should translate to different degrees of subjective deviance. The material used in this study is partly similar to the one used by Garcia-Marques and Mackie (1999; Exp. 2). As a target stereotype I chose that of computer programmers being relatively intelligent. Thus, the first 
study examines the perception of exemplars which are deviating in different directions from a competence related stereotype.

\subsubsection{Method}

Pretest. In order to identify the prototypical expectation regarding IQ-values of computer programmers, participants in a pre-test rated the average IQ-scores of several occupational groups. Computer programmers were judged as relatively intelligent ( $M=$ $110.44, S D=7.82)$ compared with a list of seven other occupations $\left(M_{\min }=91.39\right.$ to $M_{\max }=$ 111.94). The average rating for computer programmers was significantly higher than the overall average value of 100 mentioned in the introduction to the pre-test, $t(17)=5.66, \mathrm{p}<$ $.05, d=1.89$.

\section{Participants and Design}

Eighteen students from the University of Jena, Germany, participated in the study. The design of the study was a 3 (Exemplar: congruent, incongruent, supercongruent) $\times 2$ (Item: deviance, typicality) $\times 2$ (Order: incongruent first, supercongruent first) mixedsubjects design with the first two variables as repeated measures.

\section{Procedure}

Participants were told that a large psychometric survey had been conducted concerned with IQ-scores of different occupational groups. They were further informed that the IQ-test used in this survey was standardized in a way that 100 would occur as the overall average value and $68 \%$ of the population would end up with a score between 85 and 115 . Participants were asked to answer two questions related to three different results which have ostensibly occurred in this survey for computer programmers. The first result was a congruent one ( $I Q=110)$. Half of the participants judged a supercongruent computer programmer (IQ = 138) next and then an incongruent one $(I Q=82)$. The other half of the participants judged the deviant exemplars in the reversed order. All three scores were introduced with the following sentence: "Imagine a computer programmer with an IQ-score of 110 (or 138, or 82, respectively)". First, participants had to indicate on a 9-point scale ranging from 1 (not at all) to 9 (completely) how much this person deviates from the majority of computer programmers. High values indicate stronger deviance. Secondly, participants had 
to rate on a similar 9-point scale how typical this person is for computer programmers in general. High values indicate higher perceived typicality.

\subsubsection{Results and Discussion}

How are supercongruent exemplars perceived? Results suggest that participants perceive an extremely intelligent member of a group that is stereotypically seen as intelligent as deviating from the rest of the group but still as more typical than an unintelligent group member. Answers to the deviance item were recoded such that higher values indicate less perceived deviance. A 3 (Exemplar) $\times 2$ (Item) $\times 2$ (Order) mixed analysis of variance (ANOVA) with the first two factors as repeated measures revealed that order had no impact on the results, all $F_{\mathrm{S}}<1.77, p \geq .20$, and, therefore, means in Table 1 are depicted independent of order. The analysis yielded a significant effect of exemplar, $F(2,15)=25.45, p<$ $.05, \eta^{2}=.77$. However, most importantly, this effect was qualified by a significant interaction with item, $F(2,15)=5.01, p<.05, \eta^{2}=.40$. To understand this effect, simple comparisons between the exemplar conditions were carried out adjusting for multiple comparisons with sidak adjustment. Table 1 also displays the results of these comparisons. One comparison did not show a significant difference: the one between perception of deviance of an incongruent $(M=2.44)$ and a supercongruent exemplar $(M=3.50), p=.40$. This result indicates that participants perceived exemplars who deviated in different directions as equally deviating from the majority. The fact that a significant difference between the deviance of a congruent $(M=6.78)$ and supercongruent exemplar appeared, $p<.05$, points to an actual difference between supercongruent and congruent exemplars. The supercongruent exemplar was perceived as more deviating than the congruent exemplar. However, although both the incongruent and supercongruent exemplars were perceived as equally deviating, their typicality was judged differently. The supercongruent exemplar $(M=4.39)$ was judged as more typical than the incongruent exemplar $(M=1.94)$, but still less typical than the congruent exemplar $(M=7.22)$, both $p s<.05$. A second set of simple comparisons with the same adjustment revealed that only for the supercongruent exemplar did typicality and deviance judgments differ significantly from each other, $p<.05$. The two judgments, however, were marginally significant in case of the incongruent exemplar, $p=.06$.

In order to underscore the results of the simple comparisons a 2 (incongruent vs. supercongruent) $\times 2$ (deviance vs. typicality) ANOVA was additionally conducted. It was as- 
sumed that judgments for incongruent and supercongruent IQ-scores would differ on typicality but not on perceived deviance. The analysis yielded the expected interaction, $F(1,17)$ $=9.48, p<.05, \eta^{2}=.36$. This interaction was due to the fact that the two exemplars were judged differently in terms of typicality, whereas no difference appeared on perception of deviance.

Table 1: Means and standard deviations on perceived deviance and perceived typicality of incongruent, congruent and supercongruent exemplars (Study 1)

\begin{tabular}{lccccc}
\hline $\begin{array}{l}\text { Dependent } \\
\text { measure }\end{array}$ & ic & cong & sc & cong - ic & cong - sc \\
\hline deviance & 2.44 & 6.78 & 3.50 & 4.35 & 3.28 \\
& $(1.98)^{\mathrm{a}}$ & $(2.39)^{\mathrm{a}, \mathrm{b}}$ & $(2.48)^{\mathrm{b}}$ & & \\
typicality & 1.94 & 7.22 & 4.39 & 5.28 & 2.83 \\
\hline
\end{tabular}

Note. $\mathrm{N}=18$. ic $=$ incongruent; cong $=$ congruent, and $\mathrm{sc}=$ supercongruent. Higher scores represent less deviance and more typicality. Means in one row or column with the same index are significantly different from each other with sidak adjustment.

To sum up, the results of Study 1 confirmed that a difference exists between congruent and supercongruent exemplars. People are able to detect a deviance in direction of the stereotype as much as they detect a deviance in the opposite direction. The paradigm of the present study allowed for precise control over the degree of deviance from the central tendency of incongruent and supercongruent exemplars in that IQ-values deviated to the same amount from the congruent exemplar. Thereby, a comparison between the perception of incongruent and supercongruent exemplars seems to be justified. Although the objective deviance from the prototype was the same for both exemplars, participants perceived the supercongruent exemplar as more typical than the incongruent exemplar. The same objective amount of deviance seems to correspond to different degrees of subjective deviance, which is reflected in the typicality ratings.

Given that the results of Study 1 demonstrated this difference between the two different kinds of deviating exemplars, the assumption that their impact on the central tendency of the stereotype might differ as well ought to be addressed in the next studies. I argued in the theoretical introduction of this thesis that supercongruent exemplars should be used to accentuate differences between groups. Study 2 was designed to test this hypothesis. 


\subsection{Study 2: Accentuation within Stereotype Change}

\subsubsection{Introduction}

Previous studies on the accentuation principle typically used non-social categories (e.g., Krueger et al., 1989) or artificial, social categories (Krueger \& Rothbart, 1990, Exp. 3). Participants were confronted with a long list of exemplars which deviated in one or the other direction. Memory processes were assumed to be responsible for the accentuation effect. Because supercongruent exemplars are likely to be quite extreme, it is rather unlikely to assume that someone will encounter several supercongruent exemplars at once. Study 2 was designed to test the accentuation principle with one deviating exemplar, and within a similar paradigm Garcia-Marques and Mackie used to study stereotype change (1999; Exp. 2). The present study particularly aimed at studying the impact of super - and incongruent exemplars on the difference between two groups with contrasting stereotypes. The stereotype of computer programmers as being relatively intelligent was used again. As a measure of central tendency Garcia-Marques and Mackie (1999) asked participants to generate IQ scores for a random sample of members of the target category. Participants in their study and the present study received a first score of one computer programmer as an example. This score was either congruent, incongruent, or supercongruent. In contrast to the study of Garcia-Marques and Mackie, participants in the following study additionally had to generate IQ-scores for a comparison category with an opposing stereotype (house painters). Thereby, the effect of the exemplar on the perceived difference between the two groups could be investigated. Supercongruent exemplars should lead to an increase in difference, while incongruent exemplars should not affect the difference.

\subsubsection{Method}

\section{Participants and Design}

Fifty-three students from the University of Jena, Germany, participated in the experiment. They received a chocolate bar for participation. Three conditions varied between 
participants, namely the direction of deviance with an incongruent, congruent, and supercongruent exemplar ${ }^{10}$.

\section{Procedure}

Participants were told that the study was about the ability to deal with numerical concepts. The problem they had to face concerned IQ scores. Introduction to the IQ-test was the same as in Study 1. Furthermore, participants received a list of 30 names which were ostensibly randomly sampled from an exhaustive list of the employment union of computer programmers. Participants were asked to generate IQ-scores that could plausibly result if 30 members of that group were randomly sampled. The first IQ score was given as an actual example and constituted the critical manipulation ( 82 for an incongruent, 110 for a congruent, and 138 for a supercongruent computer programmer). Both deviating values were lying outside the range for computer programmers generated in the pretest. Next, participants had to perform the same task on the same page for a list of 30 house painters (comparison category). No first score was presented for this category. After performing this task, all participants had to rate the intelligence of computer programmers on a scale ranging from 1 (not at all intelligent) to 9 (extremely intelligent).

\section{Dependent measure}

In order to test the impact of the exemplar on the perceived difference between the groups, one measure of accentuation was created by averaging the generated IQ scores for computer programmers and averaging the generated IQ scores for house painters and creating a difference score. Additionally, one item captured the central tendency of the target category, namely the intelligence rating.

\subsubsection{Results and Discussion}

Four participants were excluded from the analysis. One did not estimate IQ-values for the comparison category and three were identified as outliers on the criterion of $z>2$ on

\footnotetext{
${ }^{10}$ The pretest was the same as in Study 1. I chose house painters as comparison category $(\mathrm{M}=91.38, \mathrm{SD}=$ 9.04) because they were approximately equidistant from the standardized IQ average of 100. Participants furthermore rated the expected minimum and maximum IQ-scores for those groups (Min (programmers) $=$ 93.05; Max (programmers) $=125.27$; Min $($ painters $)=77.22$; Max $($ painters $)=108.61)$. Dispersions were of similar size.
} 
the intelligence rating.

\section{Difference score of generated IQ scores}

The question is whether supercongruent and incongruent exemplars have a different impact on the perceived differences between social categories. As Table 2 reveals, supercongruent exemplars do, in fact, change the perceived difference between two categories more than incongruent exemplars. To assess the impact of the first score on the difference measure, a one-way ANOVA was performed comparing the 3 first-score conditions. The analysis yielded a significant main effect, $F(2,46)=5.83, p<.05, \eta^{2}=.20$. Means are depicted in Table 2. Simple comparisons with sidak adjustment showed that participants increased the difference between the two categories when confronted with a supercongruent first score $(M=15.66)$ compared with the congruent control condition $(M=7.20), p<.05$. An incongruent first score, on the contrary, did not lead to a decrease in the difference between the two groups $(M=7.40), p>.05$.

Table 2. Means and standard deviations on difference score (IQ-estimations for both groups) and trait judgment for the target category after confrontation with either an incongruent, congruent, or supercongruent exemplar (Study 2).

\begin{tabular}{lccccc}
\hline $\begin{array}{l}\text { Dependent } \\
\text { measure }\end{array}$ & ic & cong & sc & cong - ic & cong - sc \\
\hline difference & 7.40 & 7.20 & 15.66 & -0.20 & -8.46 \\
& $(6.40)^{\mathrm{a}}$ & $(5.72)^{\mathrm{b}}$ & $(10.99)^{\mathrm{a}, \mathrm{b}}$ & & \\
trait judgment & 6.65 & 6.93 & 7.53 & 0.28 & -0.60 \\
& $(1.66)^{\mathrm{c}}$ & $(0.83)^{\mathrm{d}}$ & $(0.74)^{\mathrm{c}, \mathrm{d}}$ & 0.28 &
\end{tabular}

Note. $\mathrm{N}=49$. ic = incongruent; cong = congruent, and $\mathrm{sc}=$ supercongruent. Higher scores represent greater difference and more stereotypicality for target category. Means in one row with the same index are significantly different from each other with sidak adjustment.

\section{Central tendency by trait ascription}

To assess the impact of the critical score on the judgment of computer programmers' intelligence, the same ANOVA as above was computed. The analysis yielded a marginally significant main effect, $F(2,46)=.2 .38, p=.10, \eta^{2}=.09$. As means in Table 2 show, computer programmers' intelligence was judged to be lowest when an incongruent critical score was depicted $(M=6.65)$, while it was highest when a supercongruent score was given $(M=$ 
7.53). Ratings in the congruent condition fell in-between $(M=6.93)$. Two t-tests for independent samples revealed that the supercongruent condition was different from the congruent condition, $t(30)=2.16, p<.05, d=.77$, while the incongruent condition did not differ from the congruent condition, $t<1$.

The results of Study 2 confirmed the hypothesis: Presenting participants with an incongruent first score did not decrease the difference between a target and a comparison category, whereas a supercongruent first score led participants to increase the difference in IQ estimation between the two categories. By creating difference scores, I wanted to demonstrate the accentuation effect due to a single deviating exemplar.

Equivalently, trait ratings became more extreme after the presentation of a supercongruent first score while no change occurred for an incongruent first score. However, on the trait judgment task the effect was rather small. But, although people generally try to avoid the extremes of a scale (Cronbach, 1950), it was still possible to detect polarization instead of change in the opposite direction.

Although this paradigm has been used in stereotype change research before, one might argue that at least the results on the generated IQ scores can be better explained in terms of anchor effects (e.g., Mussweiler \& Strack, 1999). However, the anchoring approach can not explain the difference between the supercongruent and incongruent condition. This difference must be due to a differential usage of the two anchors. In further studies I wanted to make use of other means to capture stereotype change which might be more typical for the research on stereotype change.

\subsection{Study 3: Stereotype Change Following In- and Supercongruent Ex- emplars}

\subsubsection{Introduction}

Study 3 aimed at demonstrating the effect of direction of deviance on perceived typicality and stereotype change within one design. The former studies relied on a competence related stereotype which was positive. In this study, a warmth related stereotype which has a rather negative connotation (i.e., lack of warmth) was used. A pre-post design was implemented. Hence, the experiment consisted of two sessions: a first one to assess the 
stereotype and a second one containing exemplar information followed by a second assessment.

\subsubsection{Method}

\section{Pretest}

A pretest was conducted in order to confirm the assumption that students of computer science (target category) are perceived as lacking warmth whereas social science students (comparison category) are perceived as relatively warm. Pretest-participants were requested to estimate the results of an ostensible survey on job-qualifications of students with different majors. Participants had to make judgments related to four subtests concerned with social competence (social orientation, capacity for teamwork, helpfulness, sociability). Each attribute was accompanied by a scale from 0 (very low on that attribute) to 100 (very high on that attribute) on which they had to indicate how many points an average student of computer science and an average student of social science would gather on a test measuring that attribute. Fifteen students from the University of Jena, Germany, served as pretest participants. To test the difference of the resulting ratings a 4 (Attribute) $\times 2$ (Group) withinsubjects ANOVA was conducted. The analysis revealed a significant difference for group, $F(1,14)=17.06, p<.05, \eta^{2}=.55$. Students of computer science were perceived as less warm $(M=47.82)$ than students of social science $(M=74.35)$. Yet, a significant interaction between group and attribute indicated that the effect might differ according to attribute, $F(3,12)=19.84, p<.05, \eta^{2}=.83$. Four separate repeated measures analyses for each attribute showed that the difference was significant on all attributes except for helpfulness, $F(1,14)=1.43, p=.25, \eta^{2}=.09$. However, mean values indicated that the pattern does not differ essentially from the other attributes $\left(M_{\text {social science }}=69.14\right.$ vs. $\left.M_{\text {computer science }}=59.00\right)$.

\section{Participants and Design}

One-hundred-twenty students from the University of Applied Science Jena, Germany, participated in the time 1 and forty-eight students in the time 2 data collection, two weeks later. ${ }^{11}$ They were all attending a course for business administration students. At

\footnotetext{
${ }^{11}$ The difference in sample is due to the fact that participants were randomly assigned at time 2 to two different studies and just one study is presented here. Questionnaire at time 1 was the same for all participants.
} 
both measurement points participants received a chocolate bar for participating and they could take part in a lottery for book vouchers. The design of the experiment was a 2 (Direction of deviance: incongruent vs. supercongruent) $\times 4$ (Stereotype time 2: target category/warmth vs. target category /competence vs. comparison category /warmth vs. comparison category /competence) design with the first variable being manipulated between participants and the second variable within. An effect of deviance was expected to occur only for the target category and the manipulated dimension (warmth). The stereotype measure from the first assessment was used as baseline to detect change.

\subsubsection{Procedure}

At time 1, all participants received the same questionnaire. Participants were told that the study was part of a bigger survey on job qualifications of students with different majors. Participants were told that, for reasons of simplification, only two different majors were included at this stage of the survey: students of computer science (target category) and students of social science (comparison category). At time 1 participants were asked to estimate the results of an ostensible first part of the big survey, in which the public opinion about job qualifications of different majors were supposedly assessed. They had to rate students of computer science and students of social science on traits related to warmth and competence. At time 2 participants were told that they would take part in another part of the survey, which included the confrontation with the test profile of one student. Their task was to predict his/her professional future. Participants were first made familiar with the test and the scales used in the questionnaire. They were then presented with the test results of an alumnus of computer science on different subtests related to warmth (same subtests and scales as in the pretest). Depending on the experimental condition, the results deviated either in an incongruent or in a supercongruent manner from the average pretest rating. A supercongruent deviant was created by subtracting the difference between the two groups from the value for computer science students. The results of an incongruent exemplar matched the stereotypical expectations for social science students. As a means to check for the manipulation and in order to reinforce the cover story, participants were asked to take job-related decisions for the exemplar (e.g., I think this person could fulfil a leading position) and evaluate her/him on several traits related to warmth (e.g., social competence). 
Under the pretence of gathering some control variables for the exemplar ratings, participants were asked next to judge both groups included in the survey on several traits. Attributes, rank order, and introduction to the task were the same as at time 1. Finally, participants had to answer the typicality measure. They were told that the student has to compete with alumni of her/his major in finding a job. That is why they should compare the single exemplar with other people of her/his respective group.

\section{Dependent measure}

To measure stereotype change, the same trait ratings were included at time 1 and time 2 (warm, capable, good-hearted, intelligent, sociable, ambitious, helpful, analytical, good teamworker, efficient, sensitive, motivated for achievement, outgoing). Participants were asked not to state their own opinion but rather to try to estimate the results of a public opinion poll. "I think, that society judges students of computer science to be ....". ${ }^{12}$ Judgments were made on 9-point scales, ranging from 1 (not at all) to 9 (extremely). The same ratings had to be performed for the comparison group (students of social science). The questionnaire furthermore contained three items with 9-point scales measuring perceived typicality (e.g., "How well does the exemplar fit the image of his/her group?").

\subsubsection{Results and Discussion}

Two cases of the time 2 session were excluded from further analyses. One did not fill out the trait ratings and one was detected as an outlier based on the criterion of $z>2$ on the target stereotype measure (computer science students/warmth). Due to reluctance of participants to fill in codes, cases could not be matched for time 1 and time 2. Therefore, warmth ratings of time 1 were just used as a baseline to detect change. Eight items measuring the exemplars social competence were averaged $(\alpha=.86)$ and served as a mean index of exemplars assumed warmth. Each participant rated both groups on traits concerning the warmth and competence dimension at time 1 and time 2. One index was created for warmth of computer science students at time $1(\alpha=.76)^{13}$, one for warmth of computer science students at time $2(\alpha=.80)$, one for competence of computer science students at time $2(\alpha=$

\footnotetext{
${ }^{12}$ The same way of indirectly measuring the stereotype was used by Fiske, Cuddy, Glick, \& Xu (2002).

${ }^{13}$ Since the time 1 value was just needed as a baseline to detect change, I just computed an index for warmth of computer science students.
} 
$.86)$, one for warmth of social science students at time $2(\alpha=.85)$, and one index for competence of social science students at time $2(\alpha=.85)$. The three items measuring typicality of the exemplar were averaged after one item had been recoded $(\alpha=.82)$. Higher values indicate higher perceived typicality.

\section{Manipulation check}

The mean index of exemplars' assumed warmth was submitted to a t-test for independent samples comparing the incongruent and supercongruent condition. Incongruent computer science students were perceived as more social and warm $(M=5.99)$ than supercongruent computer science students $(M=3.64), t(44)=-7.23, p<.05, d=2.13$.

\section{Typicality}

According to the hypotheses, supercongruent exemplars should be perceived as more typical than incongruent exemplars even if they share the same amount of deviance from the prototype. The t-test revealed that a computer science student with fairly high results in a social competence test (incongruent) was indeed perceived as less typical ( $M=$ 3.67) than a computer science student with extremely low results on the same test (supercongruent) $(M=5.59), t(44)=4.38, p<.05, d=1.32$.

\section{Stereotype change}

It was hypothesized that supercongruent exemplars will lead to an assimilation of the stereotype, whereas the impact of an incongruent exemplar is expected to be smaller and thus will not lead to a significant assimilation of the stereotype. I did not expect to find any impact of the manipulation on the stereotype judgment of the comparison group (social science students) and on the stereotype judgment of the unmanipulated dimension (competence). First, an ANOVA was conducted with four stereotype measures as repeated measures (warmth of computer science students, competence of computer science students, warmth of social science students, and competence of social science students) and deviance (incongruent vs. supercongruent) as independent variable. The ANOVA yielded a marginally significant Stereotype $\times$ Deviance interaction, $F(3,42)=2.29, p=.09, \eta^{2}=.14$. Separate t-tests for the four stereotype measures revealed that this interaction was due to a significant effect of deviance on the focal stereotype measure of computer science students' 
warmth. A supercongruent exemplar led to more stereotypic ratings $(M=3.84)$ compared with an incongruent exemplar $(M=4.42), t(44)=-1.79, p<.05$ (one-tailed), $d=.54$. No impact of deviance occurred for the other three stereotype measures, $t \mathrm{~s}<1.46, p \mathrm{~s} \geq .15$. So far, we just know that the exemplar affected the stereotype rating for computer science students on the warmth dimension. To test whether participants changed their stereotype in one or in both conditions, two separate t-tests were conducted, which compared the stereotypic ratings of time 2 against the mean of time $1(M=4.25, S D=1.13)$. Ratings in the supercongruent condition were significantly more stereotypic compared with this baseline value, $t(19)=-2.06, p=.05, d=.41$. Ratings in the incongruent condition did not differ from the baseline value, $t(25)=.70, p=.49$.

Table 3. Means and standard deviations on perceived typicality and stereotype judgment after confrontation with either an incongruent or supercongruent exemplar (Study 3).

\begin{tabular}{lcccc}
\hline Dependent measure & ic & sc & pre - ic & pre - sc \\
\hline typicality & 3.67 & 5.59 & & \\
& $(1.62)$ & $(1.26)$ & -0.17 & 0.42 \\
Target group warmth & 4.42 & 3.83 & & \\
& $(1.08)$ & $(1.11)$ & 4.25 & \\
Target group warmth & & $(1.13)$ & \\
(pre) & & & & \\
Note. $\mathrm{N}=$ 46. ic = incongruent; sc = supercongruent, and pre = time 1 value. Higher scores represent higher \\
perceived typicality and lower levels of stereotyping.
\end{tabular}

In sum, the results of this study support both hypotheses. They replicate the typicality effect for a different stereotype on the second central dimension, namely warmth. Additionally, it was shown that the supercongruent exemplar led to an assimilation of the stereotype, while the incongruent exemplar had no impact. Stereotypes seem to be more likely to get polarized than attenuated.

Although I created the deviating exemplars in a way that they are equally distant from the prototype, the comparability of supercongruent and incongruent exemplars is still not perfect. Confounded with the direction of deviance is a difference in extremity: Supercongruent exemplars are lying closer to the end-pole of the scale, while incongruent exemplars are rather pale. Extreme information has been demonstrated to be perceived as more diagnostic (e.g., Skowronski \& Carlston, 1989) and this in turn could lead to a stronger im- 
pact on the stereotype. Study 4 aimed at testing whether the difference in extremity is driving the effect.

\subsection{Study 4: Supercongruent or Incongruent by Means of Different Comparison Categories}

\subsubsection{Introduction}

As mentioned in the introduction, incongruent exemplars deviate in direction of a comparison category, while supercongruent exemplars deviate in the other direction, away from the comparison category. In Study 4, the same exemplar information was used for a group, about which people hold average expectations regarding competence, and super- or incongruency of the exemplar was manipulated by rendering different kinds of comparison categories salient. The comparison category was either expected to be rather incompetent or to be rather competent (for a similar paradigm see Krueger \& Rothbart, 1990). Hence, if comparison category and exemplar are situated on different sides of the continuum, the exemplar should be regarded as supercongruent, while an exemplar lying on the same side as the salient comparison category is equivalent to an incongruent exemplar. I assumed that assimilation in the case of supercongruent exemplars should be stronger than for incongruent exemplars.

A pre-post design was used again and questionnaires were matched via codes across sessions. To have more control about the pre-manipulation stereotype and to decrease the effect of social desirability concerns, I decided to use the stereotype of an artificial group. Hence, the experiment consisted of a stereotype formation phase in the pre-manipulation session. The same stereotype formation phase was administered in the second session to refresh memory and was followed by a stereotype change phase.

\subsubsection{Method}

\section{Participants and Design}

One-hundred-twelve students from the University of Jena, Germany, participated in the time 1 data collection on the campus of the university. Participants were told that the study consisted of two parts of which the second one would take place online within the 
following two weeks. One-hundred-and-five students participated in time 2 data collection. Ninety-one cases could be matched successfully over the two measurement points. At time 1 participants received a chocolate bar for participating and at time 2 they could take part in a lottery for book vouchers. The design of the experiment was a 2 (Exemplar: low vs. high in competence) $\times 2$ (Comparison group: low vs. high in competence) between-subjects design.

\section{Procedure}

Participants were told that the study was on impression formation and their task would be to form an impression about a certain group or a certain person belonging to one group.

Session 1: Stereotype formation phase. First, participants were introduced to three groups which had been given artificial labels, like group "Green", group "Blue", and group "Red". Allegedly, members of all three groups had been asked about their habits and typical characteristics. Participants were given a set of sixteen randomly sorted cards each containing a question about preferred activities, habits or characteristics and three bar charts indicating how much members of each group on average like that activity or show that characteristic. Six questions were such that a high percentage indicates high competence (e.g., "Would you refrain from holidays in order to take part in further education?"), six were such that a high percentage indicates incompetence (e.g., "Did you take extra lessons in school?"), four questions were neutral regarding competence (e.g., "Do you like to go to the swimming pool?"). By means of the bar charts group "Blue" should leave the image to be rather incompetent, group "Red" should seem to be very competent, while group "Green" was placed on all competence-related items around the midpoint. Participants were asked to go through the cards individually once or twice till they had formed an impression of the three groups. After having read the cards, participants were told that the study would further deal with two of the three groups. All three groups were presented at the very beginning of the study so that group "Green" was really perceived as average on competence. The subsequent selection of groups depended on the experimental condition participants were in: either group "Red" (high) and group "Green" (average), or group "Blue" (low) and group "Green" (average). Next, participants were asked to write a paragraph-long description about each of the two groups the study would be dealing with. This was done to solid- 
ify their impressions. Participants then rated group "Green" (focal group) on thirteen 9point scales asking participants how much each trait was true for members of that group with response options from 1 (not at all true) to 9 (very much true). The traits included seven competence related traits and six warmth related traits (as in Study 3). The same task had to be fulfilled for the second group ("Red" or "Blue" depending on the condition). Participants were thanked and reminded of session two before they left the lab.

Session 2: Stereotype formation phase. The second session was conducted online. Students who took part in the first part were invited via email to take part in the second session as well. Exactly the same procedure as in Session 1 was repeated to refresh participants' memory about the three groups. They went through all the cards, describing the three groups and were asked to briefly note their impression of the two groups the study would be dealing with.

Session 2: Stereotype change phase. Participants were told that members of both groups ("Red" and "Green" or "Blue" and "Green") were tested on several competencerelated tests. By means of a wheel of fortune the results of one member of one of the two groups would be selected and presented to the participant. Actually, it was always a member of group "Green" whose test results were depicted. Test results were presented for three subtests related to the competence dimension (problem solving abilities, ambition, comprehension) on scales ranging from 0 (very low) to 100 (very high) points. Dependent on the condition, this individual had rather high results (between 80 and 100 points) or low results (between 0 and 20 points) in all three subtests. Low and high results were equally distant from the midpoint of the scale. After this presentation, participants were asked to answer the dependent variables. First, they completed the same trait ratings as in Session 1 for both groups. Following that, they answered three items measuring the perceived typicality of the exemplar (e.g., "How typical is this member for his/her group?") on scales ranging from 1 to 9. As a manipulation check participants had to rate exemplars' competence on two items (intelligent, analytical) with the same type of scale.

\subsubsection{Results and Discussion}

Two items measuring exemplars' competence were highly correlated and therefore averaged $(r=.93, p<.05)$. Eleven cases distributed equally over all four conditions were excluded from further analyses based on their ratings of exemplars' competence: Either 
they had rated a high exemplar below the midpoint of the scale or a low exemplar above the midpoint of the scale. Each participant rated both groups on traits concerning the warmth and competence dimension at time 1 and time $2 .{ }^{14}$ Two indices were created: one for the competence of group "Green" at time $1(\alpha=.77)$ and one for the competence of the same group at time 2 after the manipulation $(\alpha=.97)$. Three typicality items were averaged after the first two items had been recoded $(\alpha=.87)$. Higher values indicate higher perceived typicality.

For all analyses except the manipulation check the design was treated as one with two conditions. One condition represents supercongruent combinations (low exemplar in case of a high comparison category or high exemplar combined with a low comparison category) and one condition represents incongruent combinations (low exemplar in case of a low comparison category or high exemplar in case of a high comparison category). The derived variable was called "deviance".

\section{Manipulation check}

To check whether the perception of group „Green“ differed according to the comparison category, the competence ratings at time 1 were compared between participants in the condition with group "Blue" salient and participants in the condition with group "Red" salient. A t-test for independent samples yielded a marginal significant difference, $t(78)=$ $1.69, p=.10, d=.38$. When group "Blue" was salient, group "Green" was judged slightly more competent $(M=5.30)$ compared with the condition when group "Red" was salient, $(M=5.01)$.

To check whether the manipulation of group "Blue" and "Red" worked as intended, a t-test for independent samples was carried out on the index of comparison groups' competence at time 1. The analysis showed that participants judged group "Blue" $(M=3.45)$ as less competent than group "Red" $(M=8.07), t(78)=12.41, p<.05, d=2.79$. Both means differed significantly from the midpoint of the scale, both $t \mathrm{~s} \geq 4.94, p \mathrm{~s} \leq .05, d \mathrm{~s} \geq .77$.

The index of exemplar's assumed competence worked as a manipulation check and was submitted to a two-way ANOVA with position of exemplar and position of comparison

\footnotetext{
${ }^{14}$ I did not expect an impact on the comparison category ratings and on the non-manipulated dimension (cf. Study 3). For simplification, results on these variables are not reported for the present study. They were mainly included in the questionnaire for ecological validity and to increase reliability for the warmth ratings.
} 
category as independent variables. The analysis yielded a significant impact of "position of exemplar", $F(1,76)=428.47, p<.05, \eta^{2}=.85$. Exemplars with high test results were perceived as more competent $(M=7.21)$ than exemplars with low test results $(M=2.62)$. Comparison group had neither alone nor in interaction any impact on perception of the exemplars' competence, both $F \mathrm{~s} \leq 2.10, p s \geq .15$ (exemplar high: $M_{\text {supercongruent }}=7.02$ vs. $M_{\text {in- }}$

congruent $=7.42$; exemplar low: $M_{\text {supercongruent }}=2.50$ vs. $M_{\text {incongruent }}=2.75$ ). Results indicate that Gelöscht: the manipulation of high and low test results was successful in that participants experienced them as equally extreme. The perception of the single deviating exemplar was not distorted.

\section{Typicality}

I hypothesized that supercongruent exemplars will be perceived as more typical than incongruent exemplars even if they share the same amount of deviance from the prototype. To account for any pre-test differences of group perception according to comparison category the typicality index was submitted to an ANCOVA with deviance as independent variable and the pre-manipulation stereotype as a covariate. A non-significant interaction term between deviance and the covariate pre-stereotype in a first ANCOVA shows that the presumption of equal regression slopes across conditions is fulfilled, $F<1$. The analysis yielded a significant effect of deviance, $F(1,77)=3.32, p<.05$ (one-tailed), $\eta^{2}=.04$. The covariate pre-stereotype showed no significant impact, $F<1$. Adjusted mean values are shown in Table 4. Supercongruent exemplars were perceived as more typical than incongruent exemplars $(M=4.25$ vs. $M=3.51)$.

Table 4. Means and standard deviations on perceived typicality and assimilation of the stereotype after confrontation with either an incongruent or supercongruent exemplar (Study 4).

\begin{tabular}{lll}
\hline Dependent measure & incongruent & supercongruent \\
\hline typicality & $3.51(1.84)$ & $4.25(1.75)$ \\
assimilation & $1.08(1.08)$ & $1.60(1.11)$
\end{tabular}

Note. $\mathrm{N}=80$. Higher scores represent higher perceived typicality and stronger assimilation. Typicality ratings are adjusted for time 1 stereotype ratings of group "Green". 
Because I was interested in the average assimilation effect across conditions, a change index was created the following manner: For participants confronted with a low exemplar the post manipulation stereotype measure was subtracted from the pre-manipulation measure. For participants confronted with a high exemplar the pre-manipulation value was subtracted from the post manipulation value. This assimilation index was subjected to a ttest to compare the two deviance combinations. More change was expected to occur in the supercongruent conditions. Indeed, supercongruent exemplars led to more assimilation compared with incongruent exemplars ( $M=1.60$ vs. $M=1.08), t(78)=2.11, p<.05, d=$ .47 . As two separate $t$-tests indicate both degrees of assimilation are significantly different from 0 , both $t \mathrm{~s} \geq 6.13, p s \leq .05, d \mathrm{~s} \geq 1.40$. Participants assimilated their impression of the target category to supercongruent as well as incongruent exemplars, but they did so to a higher extent in the case of supercongruent exemplars.

\section{Typicality and stereotype change}

I tested a model of stereotype change using the multiple regression mediation approach suggested by Baron and Kenny (1986) and Judd and Kenny (1981). Three conditions must be met to infer mediation. In the first step, deviance affected the proposed mediator (perceived typicality ${ }^{15}$ ), $\beta=.24, p<.05$. In the second step, deviance affected the dependent variable (amount of assimilation), $\beta=.23, p<.05$. In the third step, the proposed mediator affected the dependent variable, $\beta=.22, p=.06$, while controlling for the independent variable. When typicality was controlled, the effect of deviance was reduced, $\beta=$ $.18, p=.11$, but the difference between the two ßs (uncontrolled and controlled for typicality) was not significant at the .05 level (Sobel test; see Kenny, Kashy, \& Bolger, 1998). Thus, the results do not support the assumption of a mediation by perceived typicality.

Several other studies, have found support for a bidirectional link between stereotype change and typicality and not one causally preceding the other (e.g., Hewstone et al., 2000; Hewstone \& Hamberger, 2000; Maurer et al., 1995). To examine the possibility of mediation in the reversed direction (from assimilation to perceived typicality), typicality was regressed on deviance, controlling for assimilation. This analysis showed a similar pattern. The effect of deviance on typicality was slightly reduced when controlling for assimilation,

\footnotetext{
${ }^{15}$ The mediator was log-transformed before the analysis because a scatter-plot indicated that the relationship between the mediator and the dependent variable was non-linear.
} 
$\beta_{\text {uncontrolled }}=.24, \beta_{\text {controlled }}=.19$, but the difference between $\beta$ s was not significant at the .05 level (Sobel test).

To sum up, the results of Study 4 confirmed the hypotheses of higher perceived typicality and greater assimilation in case of supercongruent exemplars compared with incongruent exemplars. In this study, comparability of incongruent and supercongruent exemplars was increased, but the findings of Study 1, 2 and 3 were nevertheless replicated. The results of this study strengthen the assumption that accentuation principles are responsible for the effect. The difference in the amount of assimilation, however, does not seem to be explained by perceived typicality.

With this study, I wanted to exclude the possibility that the difference in extremity of the information and thereby differential diagnosticity is responsible for the effect. One might argue that even Study 4 cannot totally rule out this explanation because dependent on the comparison category the same exemplar differs more or less from the grand mean of all salient stimuli. The same exemplar differs more from all other salient stimuli, when the comparison category renders it supercongruent. However, if participants had perceived supercongruent information as more diagnostic than incongruent information, exemplar judgments should have differed dependent on the kind of comparison category. A nonsignificant interaction on exemplar judgment makes this alternative account less plausible.

\subsection{Discussion of Part I}

With this line of studies, I aimed at investigating how direction of deviance influences the central tendency of a stereotype. While researchers within social psychology have already gained some insights into the effect of incongruent exemplars on stereotypes, evidence about the impact of extreme, overly stereotypic exemplars, called supercongruent, is relatively scarce. So far stereotypes appeared to be quite resistant to change. I argued that this could be an effect of focusing on just one kind of deviance, incongruency. Including the other kind of deviance, supercongruency, into the research on stereotype change, might leave us with a different picture.

Incongruent and supercongruent exemplars with the same amount of objective deviance from the prototype were compared. The main assumption was that the same objective deviance will have a different subjective meaning. Based on the meta-contrast principle, it 
was hypothesized that supercongruent exemplars will be perceived as more typical than incongruent exemplars. Furthermore, I assumed, based on the higher perceived typicality and the accentuation principle, that supercongruent exemplars will lead to stronger assimilation of the stereotype than incongruent exemplars. The four studies presented largely confirmed these hypotheses. The findings were replicated for different stereotypes related to the warmth and competence dimensions. By using stereotypes with opposite valence the possibility that the effects are just due to differences in valence could be ruled out. By using warmth and competence related stereotypes, generalizability of the findings was increased.

The results of Study 1 are in line with the idea that deviance exists in two versions and that people are able to detect the well known incongruent deviance but also the introduced supercongruent deviance. Moreover, although equally distant from the prototype, the two exemplars in Study 1 were judged differently when it came to perceived typicality. The findings can be, on the one hand, regarded as a precondition for this line of research because they indicate that deviance exists in two versions (incongruent and supercongruent). On the other hand, they confirmed the first hypothesis of higher perceived typicality in case of supercongruent exemplars compared with incongruent ones. For Study 2, a paradigm was used which has been applied before within stereotype change research. By adding the salience and ratings of a comparison category I aimed at demonstrating accentuation principles. Participants used the supercongruent information to increase the difference between two groups, while incongruent information had no impact. This is one hint that supercongruent exemplars are more likely to affect the central tendency of a stereotype than incongruent exemplars. Study 3 was carried out to test this more directly. And indeed, the presence of a supercongruent exemplar affected the stereotype more than the presence of an incongruent exemplar. At the same time supercongruent exemplars were again rated as more typical than incongruent exemplars. A fourth study was carried out to exclude the possibility that this effect is driven by the greater extremity of supercongruent compared with incongruent exemplars. The aim of Study 4 was to make the two deviating exemplars more comparable. Therefore, direction of deviance was manipulated for the same exemplar by varying the salient comparison category. Either it was one lying on the same side of the continuum as the exemplar implying an incongruent relation, or it was one situated on the opposite side, entailing a supercongruent relation. Both, the typicality and the stereotype 
change hypotheses were confirmed within this paradigm. Globally, the data provides unequivocal evidence that deviant exemplars are likely to influence our stereotypes, however, direction of deviance matters. Stereotypes are not as "stubborn and resistant to change" as believed so far (Allport, 1954, p. 17).

I assumed that the stronger impact of supercongruent exemplars on the stereotype is at least partly due to their greater perceived typicality. Results in Study 4, however, provided no evidence for mediation. Evidence for a reverse mediation, which has been proposed by several researchers, is also not supported by the present data.

Results related to perceived typicality as a mediator of stereotype change are quite mixed in stereotype change research so far. Not all studies tested (or reported) the mediational hypothesis, some could provide evidence for perceived typicality as a mediator of change (e.g., Johnston \& Hewstone, 1992; Yzerbyt et al. 1999), and others provided evidence for the reverse mediation (e.g., Hewstone \& Hamberger, 2000; Maurer et al., 1995). Some have argued that typicality and stereotype change probably occur simultaneously without one causally influencing the other (e.g., Hewstone \& Hamberger, 2000; Hewstone et al., 2000). Richards and Hewstone assumed that perceived typicality "rather covaries with than mediates change" (2001, p. 55). Within all that research typicality has been manipulated and measured in rather different ways. Some have manipulated typicality via other stereotypical attributes besides the one on which the exemplar is incongruent, while others have varied typicality by means of the extremity of deviance. For the latter way of conceptualizing typicality the relation between typicality and stereotype change seems to be rather complex. Rothbart (1996) has already pointed to this contradiction: "On the one hand, the more disconfirming an individual exemplar's behavior is of the group stereotype, the greater potential force exerted on the stereotype to change. On the other hand, stereotype disconfirming information, associated with an exemplar, also decreases the "goodness of fit" between exemplar and category, reducing the likelihood that the exemplar will be activated" (p. 308). Hence, the relation between perceived typicality and stereotype change might not be as straight forward as we sometimes assume.

Another problematic issue concerning the relation between perceived typicality and stereotype change is the order of measurement. So far, typicality was measured after the stereotype because I wanted to avoid to draw participants' attention towards the deviance of 
the exemplar. In the second empirical part of this dissertation it will be demonstrated that especially for supercongruent exemplars the order of stereotype and typicality measurement is influential. However, measuring the mediator after the outcome variable is less than optimal (Kenny et al., 1998, p. 262).

The present results are rather worrying, because they imply that stereotypes are very likely to get more and more extreme. The latitude of acceptance for deviating exemplars seems to be larger in one than in the other direction. And even if people are able to detect the deviating nature of supercongruent exemplars they are ready to generalize from them to the group as whole. Additional studies should address how subtyping processes can be triggered in case of supercongruent exemplars to prevent stereotypes from becoming more and more polarized. Part II and Part III will deal with this question. By testing a moderation hypothesis, Part II will additionally help to clarify the underlying mechanism of the presented effects. 


\section{Part II: Investigating the Underlying Process (Moderator 1: Mind the Deviance)}

\subsection{Introduction}

An asymmetry in the processing of deviating information has become apparent within the first part of this dissertation: Whereas incongruent information usually is not generalized to the stereotype of a category, a supercongruent deviance is likely to lead to an assimilation of the stereotype whereby stereotypes become polarized. The question remains, why does this asymmetry occur? One potential explanation for the differential impact of supercongruent and incongruent information is provided by social judgment models, like the selective accessibility model (SAM; Mussweiler, 2001; 2003; Mussweiler \& Strack, 2000). SAM makes several assumptions about the impact of accessible information during the judgment of the self or of others. Like the inclusion/exclusion model (Schwarz \& Bless, 1992) SAM also explains when a judgment is likely to be assimilated to and when it is likely to be contrasted away from the present context. It has been assumed that assimilation occurs if judges engage in a process of similarity-testing, and contrast results if they engage in dissimilarity testing. Both kinds of hypotheses were assumed to render different kinds of knowledge about the judgmental target accessible. Whether a judge engages in similarity or dissimilarity testing depends on several moderators. One of those is psychological closeness (Mussweiler, 2003). If target and standard are perceived as close, assimilation is more likely to occur, whereas contrast should result when they are not.

I argue for more psychological closeness between a supercongruent exemplar and the prototype than between an incongruent exemplar and the prototype. The deviance between a supercongruent exemplar and the prototype should be perceived more as a quantitative deviance (cf. chapter 3). Exemplar and prototype share the same attributes or features, they just differ in extremity. Incongruent exemplars, on the contrary, do not possess the attribute in question. Therefore, the deviance implies a qualitative difference. Presumably, a qualitative difference is easier and more automatically recognized and leads to the perception of less psychological closeness than a quantitative difference. 
As a consequence, supercongruent exemplars might trigger more similarity testing because they are perceived as qualitatively similar to the category, whereas perceivers might engage in a process of dissimilarity testing when exposed to an incongruent exemplar because of the qualitative difference.

If this assumption holds, any manipulation that draws people's attention to the deviance, should render assimilation of the stereotype to a supercongruent exemplar less likely to occur. Asking people to reflect upon the difference between a certain exemplar and a category by letting them judge the typicality of the exemplar might be one mean to call their attention to the deviance of the exemplar and thereby prevent assimilation of the stereotype. The two studies summarized in chapter 8 were carried out to test this moderation effect.

\section{Typicality and Stereotype Judgment: Order of Measurement}

As outlined in the introduction of this dissertation, several researchers working in the domain of stereotype change or the inclusion/exclusion model have stressed the link between typicality and stereotype change (e.g., Hewstone \& Brown, 1986; Johnston \& Hewstone, 1992; Kunda \& Oleson, 1997; Rothbart \& John, 1985; Rothbart \& Lewis, 1988; Wilder, 1984; Yzerbyt et al., 1999). In most of the studies the stereotype was assessed first and typicality of the exemplar thereafter. Yet, they were arguing for a mediational role of typicality in stereotype change which suggests a measurement of the two constructs in a vice versa order. Very few studies actually varied the order of the stereotype and typicality judgment. Johnston and Hewstone (1992, Exp. 2) found no impact of the order of measurement on the stereotype rating. They concluded from that finding that participants judge the typicality of incongruent exemplars automatically (see also Fiske, Neuberg, Beattie, \& Milberg, 1987). However, another study that demonstrated an impact of famous, positive exemplars on the stereotype of an otherwise rather negatively evaluated group could show that this effect vanishes by letting participants judge the typicality of the exemplars inbetween (Bodenhausen, Schwarz, Bless, \& Waenke, 1995, Exp. 3). Authors argued that typicality ratings have made people conscious about the atypicality of the exemplars, which in turn led to an exclusion or subtyping of the exemplars. Or, as Park, Wolsko and Judd put it: "One potential problem with the typicality task is that it forces participants to explicitly 
think about the fit of confirming and disconfirming instances to the group. Thus, it may actually call attention to the poor fit" (2001, p. 331).

Study 1 of the present dissertation demonstrated that participants are able to detect the deviance of a supercongruent exemplar as well as they could judge the deviance of an incongruent exemplar. Although supercongruent exemplars are judged as more typical, their extremity and deviance is realized when attention is directed to it. But because they just pose a quantitative deviance, this difference in extremity between prototype and supercongruent exemplar might be easily overlooked. Confronted with this kind of exemplar typicality or deviance might not be judged automatically.

It is hypothesized that unless participants' focus is not directed to the deviance, they should generalize from supercongruent exemplars to the category. This generalization process, however, is expected to be impeded by making people reflect upon the deviance and extremity of the exemplar. Asking participants to rate the typicality of these exemplars should fulfil this purpose.

To sum up, I assume that the deviance of supercongruent exemplars is more unobtrusive and that participants do not judge their typicality automatically. Order of typicality and stereotype assessment should therefore have a bigger impact when dealing with supercongruent than when dealing with incongruent exemplars. Asking participants to rate the typicality of the exemplar before they judge the stereotype should prevent them from assimilating their stereotype to the supercongruent information. Study 5 was carried out to test these assumptions. With Study 6 the assumed processes were tested more directly by manipulating the focus of comparison through different kinds of typicality questions.

\subsection{Study 5: Can Typicality Judgments Impede Generalization?}

\section{$\underline{8.2 .1 \quad \text { Introduction }}$}

Study 5 compared the perception and impact of an incongruent and supercongruent exemplar for the stereotype of business administration students as being relatively competent. The supercongruent exemplar was one with very high results on competence related tests, whereas the incongruent exemplar was one with fairly low results on the same tests. The objective deviance (in test points) from the prototype (captured in a pretest) was identical for the incongruent and supercongruent exemplars. As a point of reference for change a 
control condition was included in which participants were confronted with a congruent exemplar (equalled the pretest ratings). In the pretest as well as in the main study a category with the opposing stereotype was made salient in order to hold the standard of comparison constant for all participants.

Additionally, the order of measurement has been manipulated. As a matter of fact, order of measurement was expected to influence stereotype ratings in case of supercongruent exemplars, but less so in case of incongruent exemplars. Asking participants first about the typicality of a supercongruent exemplar should prevent them from assimilating their stereotype which should occur when participants judge the stereotype right after the manipulation. No generalization of an incongruent exemplar was expected to occur whatsoever order of measurement because their typicality has been shown to be judged more automatically (Johnston \& Hewstone, 1992; Exp. 2).

As an ancillary question, the impact of the two deviating exemplars on aspects of perceived variability has been studied as well. As briefly outlined within chapter 2.2, stereotypes are not just represented by their central tendency, they are supposed to include dispersion parameters as well (e.g., Linville et al., 1989). Hamburger (1994) has argued that the dispersion parameters are more sensitive to change than the central tendency parameters. His model is based on the assumption that people mentally locate group members along an attribute dimension and that they record the frequency with which each value occurs. Even if a deviant exemplar is subtyped and located at the tails of the distribution, it should still influence a group's dispersion by stretching the distribution (see also GarciaMarques \& Mackie, 1999; Paolini et al., 2004). According to findings of Garcia-Marques and Mackie (1999) supercongruent exemplars should not affect perceived homogeneity, whereas incongruent exemplars should lead to a decrease. In short, perceived variability was expected to be influenced by incongruent exemplars only. Since their typicality was assumed to be rated automatically, which renders their impact independent of order of measurement, order was not expected to moderate this impact.

\section{$\underline{8.2 .2 \text { Method }}$}

Pretest 
A pretest was conducted in order to establish the population's stereotype about business administration (target category) and educational science (comparison category) students on the competence dimension. I assumed that business administration students would be perceived as relatively high in competence whereas educational science students would be perceived as relatively low in competence. Besides the rank order of the two groups, I was interested in the exact stereotypical expectations (i.e., the prototype) of the two categories on the competence dimension in order to create deviating exemplars. Procedure of this pre-test and the main study were similar to Study 3. Participants of this pretest learned that a survey had been conducted on a representative sample. This alleged study was supposed to assess participants' judgments of different student groups regarding five job-qualifying attributes related to the competence dimension (i.e., problem-solving abilities, competence, ambition, efficiency, comprehension). As such, participants of the present study had to guess the results of the alleged survey. ${ }^{16}$ Every attribute was accompanied by two identical scales from 0 (very low on that attribute) to 100 (very high on that attribute). One scale was to convey their guess for business administration students and one to indicate their guess for educational science students. Fourteen students from the University of Jena, Germany, served as pretest participants. A 5 (Attribute) $\times 2$ (Group) Repeated Measure ANOVA revealed a highly significant effect for group, $F(1,13)=10.92, p<.05, \eta^{2}=.46$. Globally, students of business administration were perceived as more competent $(M=57.81)$ than students of educational science $(M=44.94)$. The variable attribute and the interaction did not yield a significant impact, both $F \mathrm{~s} \leq 2.74$, both $p \mathrm{~s} \geq .09$. The pattern was the same on all the attributes, but simple comparisons with sidak-adjustment revealed that the difference between the two groups was not significant on competence, $p=.32$, and on efficiency, $p=$ .14 .

\section{Participants and Design}

One-hundred-eighty-four students from the University of Jena, Germany, took part in the main study. They were randomly assigned to one of the six conditions of a 3 (Direction of deviance: incongruent vs. congruent vs. supercongruent) $\times 2$ (Order: stereotype

\footnotetext{
${ }^{16}$ I chose this rather indirect way of measuring stereotypes in order to reduce social desirability tendencies.
} 
measures first vs. typicality measures first) between participants design. Everyone received a chocolate bar in exchange for participation.

\section{Procedure}

Each participant was assigned individually to an experimental condition when handed the booklet that contained instructions, stimulus materials, and dependent measures. After an introduction informing about the cover story, all participants were presented with the individual test results of an alumnus of business administration. The results on each subtest (problem solving abilities, ambition and comprehension) were communicated on scales ranging from 0 (very low) to 100 (very high). Depending on the experimental condition, the results either matched the stereotypical expectation for that group or deviated either in an incongruent or supercongruent way. A supercongruent deviance was created by multiplying by 1.5 the pretest difference between the two groups and adding this number to the results of the pretest. This exemplar thus showed very high results on the competencerelated subtests. The results of an incongruent exemplar were created symmetrically by subtracting this number from the category prototype ${ }^{17}$. This exemplar had fairly low results on the same subtests. Participants had to evaluate the exemplar on two attributes (intelligence, analytic abilities) in order to check the manipulation. Several other questions about the exemplar were asked in order to reinforce the coverstory (e.g., "I think tests like this are very valid in predicting the professional performance of a person."). All answers had to be given on 9-point rating scales.

Half of the participants rated the stereotype of the group next under the pretence of validating a new scale. The stereotype measure was followed by some items assessing perception of typicality. Participants were told that the student would have to compete with alumni of her/his major in finding a job. For this reason, they should compare the single exemplar with other members of the group. For the other half of the participants the order of the dependent measures was reversed. Participants were carefully debriefed at the end of the study.

\footnotetext{
${ }^{17}$ For instance, the prototypical business student was expected to achieve 62 points on "ambition". In contrast the prototypical educational science students was expected to gain 39 points on that subtest. Hence, a supercongruent business student had 96 points, while an incongruent business student had 28 points on ambition.
} 


\section{Dependent measures}

Several measures were included for the assessment of the group's central tendency, perceived group homogeneity, and perceptions of exemplar's typicality. According to the aim of this study, the main dependent variable was the central tendency stereotype measure. This measure consisted of seven competence related traits which were taken from Fiske et al. (2002). Participants rated how characteristic each of those traits was of business administration students in general. They were asked not to state their own opinion but rather to try to estimate the results of a public opinion poll. "I think, that society believes students of business administration to be ...". This sentence was followed by seven stereotype-relevant traits (capable, competent, efficient, intelligent, independent, analytic, and ambitious). Judgments were given on a rating scale ranging from 1 (not at all) to 7 (extremely). Next, participants had to indicate how similar they perceive business administration students to be to each other. Answers had to be given on a rating scale ranging from 1 (not at all similar) to 9 (extremely similar). The same ratings followed for the group of educational science students. In order to measure perceived typicality, participants had to judge how much the exemplar deviated from the majority of the group (recoded), how well the exemplar fitted the image of the group, and how typical they perceived the exemplar to be for the group. All answers to the typicality items were given on 9-point scales, with high numbers indicating higher perceived typicality.

\subsubsection{Results and Discussion}

The responses of twelve participants equally distributed over all six conditions had to be discarded from the analyses because they were identified as outliers based on the criterion of $z>2$ on the main dependent variable, namely the central tendency ratings for the target group. Two items measuring exemplar's assumed competence were averaged, $r(172)$ $=.62, p<.05$, and served as a manipulation check. Higher values indicate higher perceived competence. Each participant rated both groups on traits concerning the competence dimension ${ }^{18}$. One index was created for the competence of business administration students $(\alpha=.68)$. The three items measuring typicality of the exemplar were averaged $(\alpha=.81)$. Higher values indicate higher perceived typicality. All simple comparisons in the following

\footnotetext{
${ }^{18}$ The ratings for the comparison group were included to keep the frame of reference constant and to increase ecological validity but were not analyzed.
} 
analyses have been carried out with sidak adjustment. All means and standard deviations reported below are depicted in Table 5 .

\section{Manipulation check}

A successful manipulation would lead to the judgment of incongruent business student as being less competent than the supercongruent business student. The judgment of the congruent exemplar should fall in-between. Because the manipulation check occurred in all conditions directly after the exemplar manipulation, order should have neither alone nor in interaction any impact. Therefore, I just expected to find a main effect of kind of deviance. A 3 (Kind of deviance) x 2 (Order) ANOVA with exemplar's perceived competence as dependent variable yielded a significant main effect for kind of deviance, $F(2,166)=73.35, p$ $<.05, \eta^{2}=.47$. The incongruent exemplar was perceived as less competent $(M=4.20)$ than the supercongruent exemplar $(M=6.80)$ and the congruent exemplar fell in-between $(M=$ 5.90). All ratings differed significantly from one another, all $p \mathrm{~s}<.05$. Order had neither alone nor in interaction any impact, both $F \mathrm{~s} \leq 1.10$. Although the deviant exemplars have been constructed in such a way that they were equally different from the congruent exemplar, mean levels revealed that the difference between the incongruent and the congruent exemplar seems to be bigger than the difference between the congruent and the supercongruent exemplar. A - 12 - -1 - 12 - -1 contrast, comparing the congruent with the two deviating conditions, supports this observation statistically, $F(1,166)=4.50, p<.05, \eta^{2}=.03$. I will come back to this finding in the discussion of this study.

\section{Typicality}

It has been hypothesized that the supercongruent business student would be perceived as more typical than the incongruent business student even if they display the same objective amount of deviance from the prototype. The same $3 \times 2$ ANOVA as above was carried out and yielded a significant effect of kind of deviance, $F(2,166)=48.72, p<.05$, $\eta^{2}=.37$. The incongruent exemplar was indeed perceived as less typical than the supercongruent exemplar $(M=4.17$ vs. $M=6.04)$. The congruent exemplar was perceived as most typical $(M=6.26)$. Moreover, the analysis yielded a significant interaction between kind of deviance and order, $F(2,166)=4.62, p<.05, \eta^{2}=.05$. While order had no impact on the typicality ratings of the congruent exemplar, $p=.13$, order had a significant impact in the 
supercongruent condition, $p<.05$, and a marginal significant impact in the incongruent condition, $p=.06$. In both cases, deviant exemplars have been judged as more typical after the stereotype had been rated. When typicality was rated first, the typicality ratings of the supercongruent and congruent exemplar differed, $p<.05$, with the congruent exemplar being perceived as more typical. Yet, there was no difference when the stereotype was assessed first, $p=.43$.

The supercongruent exemplar has been clearly judged as more typical than the incongruent exemplar. This difference was independent of the order of measurement. Both of the deviating exemplars, incongruent and supercongruent ones, have been judged as more typical when participants rated the stereotype of the group first. However, the difference due to order was even bigger for the supercongruent exemplar. This could be due to an assimilation of the stereotype to the supercongruent exemplar, thereby decreasing the distance between the prototype and the deviant exemplar.

\section{Central tendency}

I expected to find an impact of exemplar manipulation on the central tendency rating of the group. Participants should assimilate their perception of business administration students to the supercongruent exemplar, which means that they are perceived as more competent than in the other conditions. This assimilation, however, should be blocked by asking participants to judge the typicality of the supercongruent exemplar in-between. Order of measurement was assumed to have less of an impact for incongruent exemplars. The same ANOVA as in the two previous sections was carried out and yielded a significant main effect for direction of deviance, $F(2,166)=5.38, p<.05, \eta^{2}=.06$. This main effect was qualified by an interaction with order, $F(2,166)=3.03, p=.05, \eta^{2}=.04$. When typicality was assessed first, stereotype ratings did not differ according to exemplar, all $p \mathrm{~s} \geq .74$. However, when the stereotype was assessed first, business administration students in general were judged as more competent after a supercongruent exemplar $(M=5.60)$ compared with a congruent $(M=5.16)$, or incongruent one $(M=4.96)$, both $p s<.05$. Ratings in the congruent and incongruent condition did not differ, $p=.51$. Just in the supercongruent conditions stereotype ratings differed significantly depending on the order of measurement, $p<$ .05 (other $p \mathrm{~s} \geq .27$ ). Ratings were more stereotypical when participants rated the stereotype first $(M=5.60)$ compared with the condition when they rated the typicality first $(M=5.26)$. 


\section{Homogeneity measure}

The same ANOVA as in the previous sections was carried out. The analysis yielded a significant main effect for direction of deviance, $F(2,166)=3.48, p<.05, \eta^{2}=.04$. Business students were judged to be less homogeneous when participants had been confronted with an incongruent business student $(M=6.08)$, and more homogenous when the exemplar was supercongruent $(M=6.87), p<.05$. Homogeneity ratings of participants confronted with a congruent exemplar fell in-between $(M=6.63)$ but did not differ significantly from one of the other two, both $p s \geq .22$. Order had neither alone nor in interaction any significant impact, $F_{\mathrm{S}}<2.74, p \mathrm{~s} \geq .10$. Mean levels are indicating a slight tendency to judge the group as more homogeneous when typicality was assessed first.

Table 5. Means and standard deviations on exemplar judgment, perceived typicality, central tendency and perceived homogeneity dependent on direction of deviance (incongruent, congruent, or supercongruent) and order of measurement (Study 5).

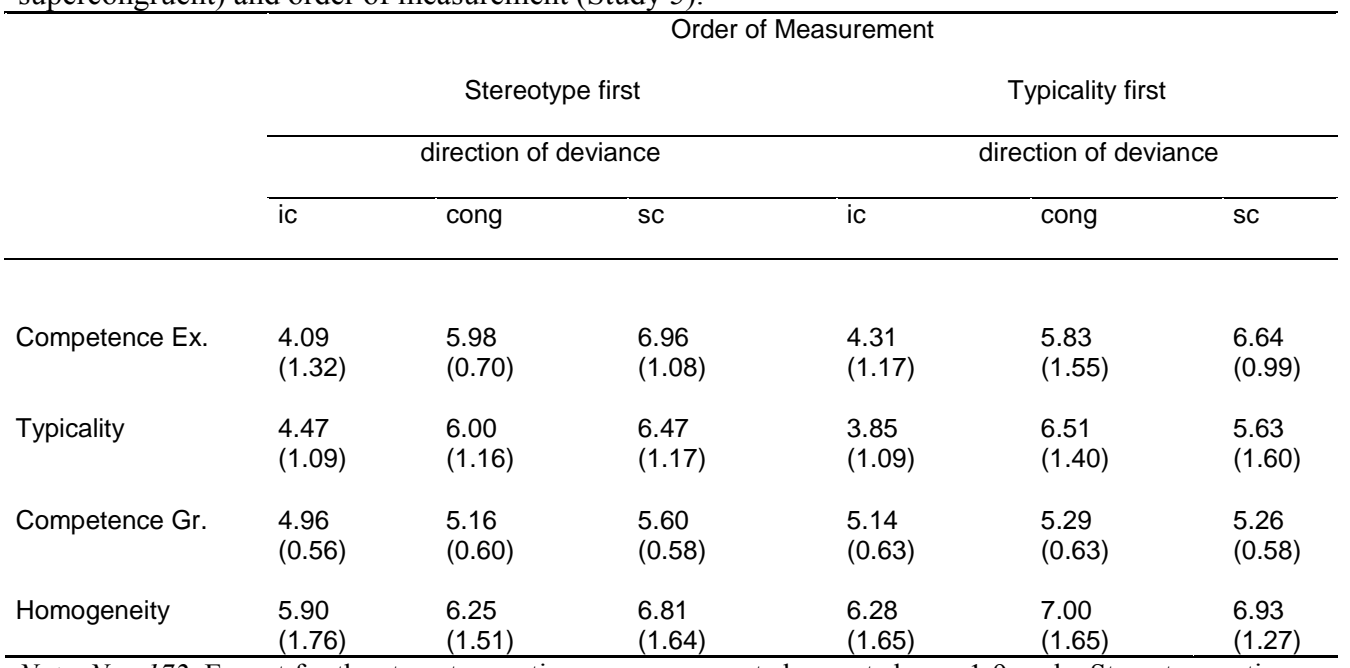

Note. $N=172$. Except for the stereotype ratings means presented are rated on a 1-9 scale. Stereotype ratings were made on a scale ranging from 1-7. Higher values indicate higher perceived competence, higher perceived typicality, higher levels of stereotypes, and higher perceived homogeneity.

To sum up, results confirmed the first hypothesis regarding typicality. A rather incompetent business student (incongruent) was perceived as less typical than a highly competent business student (supercongruent). Order of measurement influenced the perception of typicality of the supercongruent business student. He was perceived as equally typical as the congruent business student when the stereotype was assessed first. Yet, asking partici- 
pants to judge the typicality in the first place, led them to judge the supercongruent business student as less typical than the congruent one. It seems as if people are able to notice the deviance of supercongruent exemplars if their attention is drawn to it. But when they judged the stereotype in-between and assimilated the prototype to the supercongruent exemplar, perceived typicality was higher. And indeed participants seemed to have assimilated their stereotype to the supercongruent exemplar but just if they are not asked to judge the typicality in-between. Hence, as expected, in case of a supercongruent exemplar the stereotype judgement was affected by the order measurement. Order did not have a significant impact on the stereotype rating following incongruent exemplars. This is in line with earlier findings by Johnston and Hewstone (1992, Exp. 2), who argued that people spontaneously judge the typicality of an incongruent exemplar and therefore order of measurement does not have any impact. The present results on the homogeneity measure are replicating previous research by Garcia-Marques and Mackie (1999). Perceived homogeneity was lower in the incongruent condition than in the supercongruent condition. Although both levels did not differ significantly from the congruent condition, means are pointing to a higher change in the incongruent condition than in the supercongruent one.

Although exemplars were created in a way, that they were equally distant from the prototype of the category, the difference in perceived competence seems to be bigger between the incongruent and the congruent exemplar compared with the difference between the congruent and the supercongruent exemplar. A first possible explanation could be ceiling effects. The supercongruent exemplar was more extreme than the incongruent exemplar and therefore trait ratings for the supercongruent exemplar could have been subject of ceiling effects. No evidence was found for this explanation, because variance was not restricted for the trait ratings of supercongruent exemplars and answers seem to be normally distributed. A second possible explanation is based on the assumption that person perception is equally context dependent as group perception whereby the context leads either to an assimilated or contrasted judgment (e.g., Eiser, 1990; Schwarz \& Bless, 1992). Whether or not the exemplar judgment is affected by the information about the group-membership might rest on the same processes involved (and described before) in the stereotype judgment. Since typicality was rated in all conditions after the manipulation check (i.e., exemplar ratings), the findings should parallel the stereotype ratings in the stereotype first condi- 
tion. That means, the judgment of a supercongruent exemplar might be assimilated to the prototype, whereas the judgment of an incongruent exemplars might not be affected by the prototype of the category. Although this assumption cannot be tested by means of a posthoc test with a different sample, it could be examined at least whether the exemplar judgments were indeed influenced by the information about the group-membership and whether distances would be equal when no such information is provided. Therefore, I presented the same exemplars as in the main study to 30 participants without mentioning any group membership. Participants were asked to judge the competence of the exemplar in the same manner as in the main study. Deviance of exemplars was manipulated between participants. The two items (intelligent, analytical abilities) were again highly correlated, $r(30)=.61, p$ $<.05$, and therefore averaged. Two orthogonal contrasts have been created: One comparing the congruent with the two deviating conditions (C1: $-12-1)$, and the other one comparing the incongruent and supercongruent condition $(\mathrm{C} 2:-101)$. The first contrast revealed that the distance between the competence rating of the incongruent exemplar and the congruent exemplar was as big as the distance between the congruent and the supercongruent exemplar, $\mathrm{C} 1: t(27)=-.17, p=.86$. As expected, the second contrast yielded significant, $\mathrm{C} 2$ : $t(27)=-6.67, p<.05$. The supercongruent exemplar $(\mathrm{M}=7.25)$ was perceived as more competent than the congruent exemplar $(M=5.65)$, which in turn was perceived a more competent than an incongruent exemplar $(M=3.90)$. Hence, if the information was presented without categorical information, the deviant exemplars seem to be equally distant from the prototype. The pre-condition of creating exemplars with an equal distance to the prototype was successful. Presumably, the supercongruent exemplar was assimilated to the prototype in the main study. This assimilation, though, was probably not strong enough to render the supercongruent exemplar congruent otherwise no assimilation of the stereotype, no order effect, and especially no difference between the supercongruent and congruent exemplar on the exemplar judgment should have occurred.

One of the major aims of this study was to test whether an assimilation in case of supercongruent exemplars is inevitable or whether it can be blocked by drawing people's attention towards the deviance. The order effect demonstrates that drawing participants' attention towards the deviance makes generalization less likely to occur. But is it enough to let participants compare the exemplar with the category? Or has the focus of comparison 
been manipulated through the kind of typicality question? The first item in the row of typicality items asked participants to judge how much the exemplar is deviating from the prototype of the category. The selective accessibility model (Mussweiler, 2001; 2003; Mussweiler \& Strack, 2000) assumes that the nature of the hypothesis that is tested influences whether assimilation or contrast is likely to occur. Asking participants about the deviance of the exemplar, could trigger the hypothesis that exemplar and category are dissimilar. This in turn, could have prevented participants from assimilating the prototype to the exemplar. Instead, if the first item would have asked participants to judge the similarity of the supercongruent exemplar and the category, results on the stereotype measure could have been similar to those in the stereotype first condition. Asking to judge the similarity might trigger a similarity focus and thereby leading participants to assimilate the stereotype to the supercongruent exemplar. This assumption was tested within Study 6. In the absence of any typicality item, we expected to replicate the assimilation effect obtained in Study 5.

\subsection{Study 6: Can Typicality Judgments Draw Focus on Similarities or Dissimilarities?}

\section{$\underline{\text { 8.3.1 Introduction }}$}

Hewstone and colleagues tested in one study the impact of processing instructions on subtyping versus generalization of an incongruent exemplar (Hewstone et al., 2000). They demonstrated that a focus on differences leads to similar results as no focus manipulation, which is no change, whereas a focus on similarities leads to less pronounced stereotypes. This result suggests that the instruction to focus on dissimilarities seems to resemble what people are already doing when confronted with an incongruent exemplar. As mentioned before, I assume that the default process when dealing with supercongruent exemplars is similarity testing. Focus of comparison was manipulated through different kinds of typicality items in Study 6 to test this idea. The aims of the study were twofold. First, it aimed at replicating the findings of Study 5 with a different stereotype. A stereotype was chosen that is related to warmth. Study 6 makes use of the stereotype of physics students as lacking warmth. I expected to replicate the finding that the stereotype is assimilated to the supercongruent exemplar if the stereotype is assessed directly after the manipulation without participants having to judge the exemplar's typicality in-between. The second aim of 
Study 6 was to test, whether participants' tendency to generalize from a supercongruent exemplar to the category will be influenced by the kind of typicality judgment they have to make. Assimilation of the stereotype to the supercongruent exemplar should be attenuated by asking participants to rate the typicality of the exemplar in terms of deviance (i.e., dissimilarity). However, if participants have to rate the typicality in the first place but in terms of similarity, they should still assimilate the stereotype to the exemplar. An incongruent exemplar condition was not realized as the focus of this study is on the processes that are triggered by supercongruent exemplars.

\section{$\underline{\text { 8.3.2 Method }}$}

\section{Pretest}

A pretest has been conducted in order to figure out the stereotype about physics students (target category) compared with students of social science (comparison category) on the warmth dimension. The assumption was that physics students would be perceived as lacking warmth whereas social science students would be perceived as relatively warm. The procedure of the pretest was equivalent to the one of Study 5. Pretest-participants were again requested to estimate the results of an ostensible survey on job-qualifications of students with different majors, but this time on subtests related to social competence or warmth (social orientation, capacity for teamwork, helpfulness, sociableness). Seventeen students from the University of Jena, Germany, served as pretest participants. A 4 (Attribute) $\times 2$ (Group) Repeated-Measure ANOVA revealed a significant effect for group, $F(1,16)=68.40, p<.05, \eta^{2}=.81$. Physics students were perceived as less warm ( $M=$ $37.61)$ than students of social science $(M=75.95)$ over and above the single attributes. Furthermore, the analysis yielded a significant interaction with attribute, $F(3,14)=3.57 ; p$ $<.05, \eta^{2}=.43$, but simple comparisons demonstrated that the difference between the two groups was significant on all the attributes, all $p$ s $<.05$.

\section{Participants and Design}

One-hundred-fifty-two students from the University of Jena, Germany, participated in the data collection. Data of two participants was not inserted because they took part in many similar studies before, leaving a sample size of one-hundred-fifty students. All par- 
ticipants received a chocolate bar for participation and they could take part in a lottery for book vouchers. The design of the experiment was a 2 (Exemplar: congruent vs. supercongruent) $\times 3$ (Order: stereotype first vs. typicality first/similarity vs. typicality first/difference) between-subjects design.

\section{Procedure}

Procedure and cover-story were by and large the same as in Study 5. Participants were presented with the individual test results of an alumnus of physics. All results for each subtest (social orientation, helpfulness, capacity for teamwork, sociableness) were administered on a scale ranging from 0 (very low) to 100 (very high). Depending on the experimental condition of the participant, the results either deviated from the pretest value (supercongruent) or did not deviate (congruent). A supercongruent deviance was created by subtracting the difference between the two groups from the value expected for physics students leading to a lower level of social competence. Participants had to judge the exemplar on several attributes (e.g., social competence, orientation towards his associates) in order to check the manipulation. Several other questions about the exemplar were asked in order to backup the cover-story (e.g., "I think tests like this are very valid in predicting the professional performance of a person").

Two third of the participants had two rate the typicality of the exemplar before they were asked about the stereotype. In the typicality first/similarity condition the typicality item was framed in a similarity mode ("How similar, do you think, is this exemplar to other members of his/her group?"). Participants in the typicality first/difference condition had to answer the following question instead: "How different is this exemplar from the majority of his/her group?" In both exemplar conditions participants had to rate the stereotype of both groups (target and comparison) on attributes pertaining to warmth and competence next. ${ }^{19}$ Competence related traits were included to increase reliability. We did not expect the manipulation to have any impact on this dimension. Participants in the stereotype first condition answered the stereotype measure directly after the manipulation of the check. All participants were carefully debriefed before leaving.

\section{Dependent measures}

\footnotetext{
${ }^{19}$ Comparison group ratings were just included for ecological validity and were not analyzed (cf. Study 3 ).
} 
To measure stereotype change attributes were selected based on the research of Fiske et al. (2002) and were covering the warmth and competence dimension. Introduction and scales were identical to the ones used in Study 5. In addition to the competence-related traits of Study 5, several warmth-related traits (warm, good natured, companionable, helpful, capable of teamwork, sensitive, sociable) were added. Traits pertaining to the two dimensions were alternated.

\subsubsection{Results and Discussion}

Three items measuring the exemplar's social competence (e.g., "I think this person would be able to lead a team") were averaged $(\alpha=.74)$ and served as a mean index of exemplar's assumed warmth. The seven attributes capturing the warmth judgment of physics students were averaged and served as the main dependent variable $(\alpha=.72)$. Eight cases distributed over all six conditions had to be discarded from the analyses because they were identified as outliers based on the criterion of $z>2$ on the main dependent variable, the warmth rating of physics students. The six competence related traits were averaged as well $(\alpha=.77)$. All simple comparisons in the following analyses have been carried out with sidak adjustment. All means and standard deviations reported below are depicted in Table 6 .

\section{Manipulation check}

Like in Study 5 the manipulation check item (mean index of exemplar's assumed warmth) was submitted to a 2 (Exemplar) $\times 3$ (Order) ANOVA. As expected, a main effect of exemplar occurred, $F(1,136)=24.59, p<.05, \eta^{2}=.15$. The supercongruent exemplar was perceived as less warm and less suited for jobs with a lot of social contact $(M=2.39)$ compared with the congruent exemplar $(M=3.33)$. Order was not expected to have any impact, because it has not been manipulated at the time of exemplar rating, both $F \mathrm{~s} \leq 2.04$, $p \mathrm{~s} \geq .14$.

\section{Stereotype}

The supercongruent exemplar was assumed to lead to an assimilation of the stereotype as long as people are not forced to think about the typicality of the exemplar inbetween or if people are made to think about the typicality in-between but with a focus on similarities. Hence, higher levels of stereotyping (i.e., lower warmth ratings) were expected 
in these conditions compared with the congruent condition. No assimilation of the stereotype or even contrast was assumed to occur when participants were made to think about the difference between exemplar and category. The manipulations were not expected to show any effect on the non-manipulated dimension (i.e., competence). An ANOVA with repeated measures on physics students' warmth and competence yielded a significant three-wayinteraction of exemplar $\mathrm{x}$ typicality/order $\mathrm{x}$ dimension, $F(2,136)=3.15, p<.05, \eta^{2}=.04$. In order to explore the three-way interaction further, I conducted separate analyses for the warmth and the competence judgment. While no significant effects emerged for the competence judgement, all $F \mathrm{~S} \leq 1.26^{20}$, the analysis on the warmth judgment yielded a main effect of exemplar, $F(1,136)=4.69, p<.05, \eta^{2}=.03$. Physics students were judged as less warm in the supercongruent conditions compared with the congruent conditions ( $M=3.96$ vs. $M=$ 4.26). Additionally, a marginally significant effect of order emerged, $F(2,136)=3.04, p=$ $.05, \eta^{2}=.04$. Simple comparisons indicated that physics students were judged as less warm when participants had to judge the similarity between the exemplar and the category ( $M=$ 3.87) compared with those conditions, when they immediately rated the stereotype ( $M=$ $4.27), p=.06$. The predicted interaction, however, did not render significant, $F(2,136)=$ $2.06, p=.13, \eta^{2}=.03$. At least, the expected pattern emerged by testing simple comparisons: Kind of exemplar had a significant impact on the judgment of physics students warmth when stereotype was assessed first, $p<.05$, or when typicality was assessed in a similarity mode, $p<.05$ (one-tailed). No significant differences emerged when typicality was assessed in a difference mode, $p=.71$. When the stereotype was assessed first, participants judged physics students as less warm in the supercongruent condition $(M=4.00)$ than in the congruent condition $(M=4.54)$. The same difference emerged when typicality was assessed first, but in a similarity mode $(M=3.67$ vs. $M=4.09)$. No difference was observed when typicality was assessed first in a difference mode ( $M=4.22$ vs. $M=4.14)$.

Although the simple comparisons revealed the expected pattern, the two-way interaction did not yield significant. To check whether all selected warmth traits are actually loading on the same underlying factor, a principal component analysis with varimax rotation was conducted. The analysis yielded two factors with eigenvalues greater than 1.0. One was summarizing the traits "warm" and "good-natured", the other factor was summa-

\footnotetext{
${ }^{20}$ Means for competence ratings are therefore not depicted in Table 6.
} 
rizing the traits "capable of teamwork", "companionable", "helpful", "sensitive", and "sociable". According to Leach (2007) the warmth dimension can be further differentiated into morality (e.g., honest, sincere), sociability (e.g., warm, good-natured, sociable), cooperativeness (e.g., helpful), compatibility (e.g., companionable). Although his distinction does not seem to fit the results of the present factor analysis, it still suggests that warmth might consist of different characteristics. For the present results a different interpretation might be suitable. The study was framed as one concerning job-qualifying attributes. The first factor, however, consists of traits which might be less relevant in a professional context and can be summarized more as general warmth, while the second factor seems to comprise traits which are often times demanded in job advertisements and are referred to as social skills. This distinction might have been even more stressed by the competence related traits which occurred in-between. Based on the factor analysis, I created two indices, one for warmth ("warm", "good-natured", $r=.47$ ) and one for social skills ("capable of teamwork", "sociable", "companionable", "helpful", and "sensitive" ${ }^{21}, \alpha=.68$ ).

Separate analyses on these two indices revealed that results on the second factor (i.e., social skills) were fairly similar to the results on the general index: A main effect of exemplar emerged, $F(1,136)=3.56, p=.06, \eta^{2}=.03$, in the expected direction, but the interaction was not significant, $F<1$. However, results on the first factor (i.e., warmth) were in line with the predictions. Besides a main effect for typicality, $F(2,136)=5.19, p<.05, \eta^{2}$ $=.07$, the expected interaction occurred, $F(2,136)=4.19, p<.05, \eta^{2}=.06$ (see Table 6). Simple comparisons revealed that judgments differed between the congruent and supercongruent condition when typicality was assessed first with a focus on similarities, $p<.05$. Physics students were judged to be more warm in the congruent condition than in the supercongruent condition ( $M=4.52$ vs. $M=3.80$ ). The same significant pattern emerged when the stereotype was assessed first, $p=.05$ (one-tailed), with physics students being judged as more warm in the congruent condition than in the supercongruent condition ( $M=$ 4.71 vs. $M=4.17)$. A marginal significant difference in the opposite direction occurred when typicality was assessed first and was formulated in a difference manner, ( $M=4.64$ vs. $M=5.15$ ), $p=.06$ (one-tailed), providing evidence for a contrast effect.

\footnotetext{
${ }^{21}$ In order of their factor loadings (attribute with the highest factor loading first)
} 
Table 6. Means and standard deviations on stereotype judgment dependent on kind of exemplar (congruent or supercongruent) and focus of comparison (similarity, dissimilarity, default) (Study 6). Direction of deviance

\begin{tabular}{|c|c|c|c|c|c|c|}
\hline & \multicolumn{3}{|c|}{ congruent } & \multicolumn{3}{|c|}{ supercongruent } \\
\hline & \multicolumn{3}{|c|}{ order } & \multicolumn{3}{|c|}{ order } \\
\hline & $\begin{array}{l}\text { Typicality } \\
\text { first/Sim. }\end{array}$ & $\begin{array}{l}\text { Typicality } \\
\text { first/Diff. }\end{array}$ & $\begin{array}{l}\text { Stereotype } \\
\text { first }\end{array}$ & $\begin{array}{l}\text { Typicality } \\
\text { first/Sim. }\end{array}$ & $\begin{array}{l}\text { Typicality } \\
\text { first/Diff. }\end{array}$ & $\begin{array}{l}\text { Stereotype } \\
\text { first }\end{array}$ \\
\hline Warmth (general) & $4.09(0.76)$ & $4.14(0.76)$ & $4.54(0.86)$ & $3.67(0.87)$ & $4.22(0.84)$ & $4.00(0.63)$ \\
\hline F1 (warmth) & $4.52(1.27)$ & $4.64(0.90)$ & $4.71(1.14)$ & $3.80(1.08)$ & $5.15(1.38)$ & $4.17(0.91)$ \\
\hline F2 (social skills) & $3.92(0.88)$ & 3.94 (1.01) & $4.47(0.95)$ & 3.62 (1.07) & $3.85(0.93)$ & $3.93(0.89)$ \\
\hline
\end{tabular}

Note. $\mathrm{N}=142$. Mean ratings are made on $1-9$ scales. Lower values indicate stronger stereotypes.

To sum up, Study 6 shed some further light on the role of order of measurement in stereotype change and the processes that are triggered by a supercongruent exemplar. The stereotype of physics students as lacking warmth was assimilated to a supercongruent physics student, and thereby polarized, when the experimental task did not lead participants to focus on dissimilarities. Letting participants judge the deviance of the exemplar from the other members of the category fulfilled the purpose of drawing participants' attention towards the extremity of the exemplar, thereby triggering a focus on dissimilarities.

Warmth traits in this study were loading on two different factors. The expected pattern just occurred on the factor which consisted of the traits "warm" and "good-natured". On the second factor a main effect of exemplar emerged, but not the expected interaction. Results especially differed between the two indices in the condition with the manipulation to focus on dissimilarities. A repeated measurement ANOVA with both factors confirmed this observation statistically. The analysis yielded a significant interaction between factor and typicality, $F(2,136)=4.04, p<.05, \eta^{2}=.06$. Just in those conditions in which participants' focus was directed on differences, ratings on factor 1 (warmth) were higher than on factor 2 (social skills) ( $M=4.90$ vs. $M=3.89$ ), indicating that the dissimilarity focus manipulation led participants to rate physics students as quite warm (factor 1) providing evidence even for a contrast effect, whereas the social skills ratings (factor 2) do not seem to be much affected by the dissimilarity focus manipulation. The two traits which were loading on the first factor were the first two traits in the questionnaire. The manipulation with 
which a focus on dissimilarities was induced might have been not strong enough to affect all the traits. But since content and time of measurement are confounded here, I cannot draw any firm conclusions about why the expected results only occurred on one subdimension and not on the other one.

\subsection{Discussion of Part II}

Studies summarized in Part II investigated the basic process of stereotype polarization due to supercongruent information. One way of gaining further knowledge about a process is to identify those situations in which the process is interrupted and the effect does not occur. I postulated that perceivers might not be easily aware of the deviance of supercongruent exemplars because their deviance is just a matter of degree, and, hence, can be understood as a quantitative deviance. Incongruent exemplars, on the contrary, might constitute more of a qualitative deviance because they are likely to do not possess the stereotypic attribute. Based on this reasoning, I expected the default process when dealing with supercongruent exemplars to be a focus on similarities instead of dissimilarities. This in turn could lead to the assimilation process which has been demonstrated in Part I. If this assumption holds, people should be less likely to assimilate the stereotype to a supercongruent exemplar when their attention is drawn towards the deviance of the exemplar. Asking perceivers to judge the degree of an exemplar's deviance before judging the category, was assumed to make them aware of the deviating nature and, hence, should render assimilation of the stereotype to the supercongruent exemplar less likely to occur. Technically speaking, the order of typicality and stereotype measurement should have a moderating impact on the basic effect of supercongruent exemplars on the stereotype.

Study 5 and 6 replicated the results summarized in Part I in demonstrating that generalization of supercongruent exemplars is very likely to occur when the stereotype is assessed directly after the exemplar presentation. This generalization however was blocked by asking participants to judge the deviance of the exemplar from other members of the category before the stereotype was assessed. This effect appeared for two different stereotypes and seems to be independent of valence and content of the stereotype. Furthermore, when the typicality question implied a similarity judgment, generalization occurred independent of order of measurement (Study 6). The parallel effects of direct stereotype as- 
sessment and stereotype assessment after similarity judgment suggest that engaging in similarity testing seems to be the default reaction when confronted with supercongruent members of a category. On the contrary, order of measurement did not affect reactions when confronted with an incongruent exemplar (no change; Study 5). This finding replicates previous research (Johnston and Hewstone, 1992, Exp. 2; Hewstone et al., 2000) and suggests, on the one hand, that typicality of incongruent exemplars is judged automatically, and on the other hand, that incongruent exemplars are likely to trigger a focus on dissimilarities. Hewstone and colleagues have mentioned this before: "The instruction to focus on differences between exemplars should merely encourage perceivers to do what they already seem to do" (2000; p. 402).

The basic aim of the present studies was to investigate the impact of direction of deviance on the central tendency facet of a stereotype. But stereotypes probably do not just consist of beliefs about the central tendency. Several researchers pointed to the necessity of studying perceived variability as another facet of stereotypes (Hamburger, 1994; Linville et al., 1989; Judd et al., 1991). And, not just on the central tendency facet of a stereotype, also on perceived dispersion, supercongruent and incongruent led to diverging effects in Study 5. Incongruent exemplars led to higher perceived variability than supercongruent exemplars. This finding is replicating previous research (e.g., Garcia-Marques \& Mackie; 1999).

In Study 5, the assimilation of the stereotype to the supercongruent exemplar was paralleled by the finding that exemplar judgments have been also biased in a way that the supercongruent exemplar deviated less from the prototype than the incongruent exemplar. If we expect supercongruent exemplars to be processed with a focus on similarities between exemplar and prototype, an assimilation of the exemplar judgment to the prototype ought to be expected. These results open up an additional possibility how to study the processes involved in dealing with incongruent vs. supercongruent information. In contrast to stereotype judgments which are always inflicted with social desirability concerns, exemplar judgments should be less threatening to participants. Future studies might provide participants with exemplar information that is accompanied or not with information on group membership. This information should be just affecting the judgment of a supercongruent exemplar and this effect should be mediated by focus of comparison, which could be measured in an unrelated task (see for a similar procedure Mussweiler et al., 2003). 
Why do people react differently to incongruent and supercongruent information? Usually, questions like this are answered by a mediational model. But, the order effect obtained for the measurement of typicality and stereotype poses an additional reason why it might be difficult to show a mediation by perceived typicality (see also chapter 7). Usually, the mediator is conducted before the outcome variable (Kenny et al., 1998). However, this procedure is not possible in the present context because the measurement of the mediator prevents the effect. But the present studies are, yet, a first step in answering the process question. They suggest that the process might not be driven purely by a motivation to protect and strengthen the stereotype. Instead, they point to a rather cognitive process (for a further discussion on the process see chapter 10.2).

Since stereotypes often-times imply negative consequences, like discrimination, they might want to be prevented from polarization in many circumstances. The present experiments, at least, point to peoples' ability to detect a deviance in case of supercongruent exemplars. Yet, this does not seem to happen automatically. In real life it might be often difficult to make people aware of the deviance. But maybe, there are other, more practical means how people can be prevented from assimilating their stereotype to a supercongruent exemplar. In the third empirical chapter I would like to present one more study which investigates whether subtyping of supercongruent exemplars can be triggered. 


\section{Part III: The Positive Side of Subtyping (Moderator 2: Get- ting to Know the Deviant)}

\subsection{Introduction}

Studies summarized so far within this dissertation indicated that an assimilation to supercongruent exemplars is very likely to occur and is independent of the content (warmth vs. competence) and valence of the stereotype. Since many social stereotypes are rather negative, the next aim was, to learn how exclusion or subtyping of those exemplars from our picture of a social group could be reinforced.

Subtyping has been defined before as the process by that exemplars who disconfirm the stereotype are treated as exceptions that prove the rule, get mentally clustered together and do not affect the stereotype (Maurer, et al., 1995). Previous research has shown that one factor influencing whether subtyping or generalization of an incongruent exemplar is likely to occur, is the absence or presence of neutral information. Participants in a study of Kunda and Oleson (1995; Exp. 4) were presented with an unpromiscuous gay man who contradicted the stereotype of gay men being rather promiscuous (incongruent). Either participants received no additional information about the target, or the pseudo-relevant information that he works as an accountant. It turned out that participants viewed gay men in general to be less promiscuous when they received no additional information about the exemplars' profession. But generalization from the incongruent exemplar was blocked when participants were provided with information about the profession. Promiscuity of gay men was rated in this condition just like in a baseline condition. Kunda and Oleson reasoned that the subtyping attempt is either triggered by the experience of surprise or by the motivation to maintain the stereotype. In any case, people seem to use the neutral information in order to justify subtyping.

Kunda and Oleson (1995) included an overly stereotypic condition in the very same study to rule out some alternative explanations. Although their study provides evidence that generalization of supercongruent exemplars can not be blocked by providing neutral additional information, I believe a second test is worthwhile. First, because Kunda and Oleson, since they were mainly interested in the effect of incongruent exemplars, did not provide evidence that the overly stereotypic exemplar they chose was actually deviating from proto- 
typical expectations. Therefore, we do not know whether the exemplar was actually supercongruent or whether it was just congruent. Second, null-effects can occur due to several reasons. The additional information Kunda and Oleson provided was that the target works as an accountant. The picture typically painted of accountants is that they are orderly, methodical, introverted, and unsociable (see Bedeian et al., 1986). A pretest in which Kunda and Oleson tested whether a gay man who works as an accountant is perceived as more or less promiscuous than gay men in general showed no significant difference but a tendency towards gay men working as an accountant being perceived as less promiscuous. The additional information Kunda and Oleson chose might thus have been more applicable to explain away an incongruent deviance than a supercongruent one.

\subsection{Study 7: The Impact of Additional Information}

One additional aim of the present study was to introduce a new paradigm for studying stereotype change, one which is rather unobtrusive and more realistic. Participants believed that they were playing a game against several other students with whom they were ostensibly connected via the server. Their group membership was manipulated by providing participants with some information about their co-players. I manipulated the perception of exemplars warmth by giving feedback about the other players' decisions. Thereby an encounter with a supercongruent exemplar was established.

\subsubsection{Method}

The stereotype of business students as being not very social and warm was used for this study. Through feedback in a fictitious experimental game, participants were brought into contact with a supercongruent business student who behaved selfish in this game. Half of the experimental participants received the additional information about the business student that could be used to exclude the exemplar: He was said to be specialized in marketing and production management.

\section{Pretest}

In a pretest fifteen students of the University of Jena with different majors were asked to rate the social orientation of several groups of students (biologists, psychologist, law students, educational science students, and business students) on a scale from 0 (very 
low) to 100 (very high). Groups were judged differently, $F(4,11)=20.45, p<.05, \eta^{2}=.88$. Means revealed that business students were judged as the group that is the least social $(M=$ 38.67) and educational science students were judged as the group which is the most social $(M=68.07)$. Ratings for law $(M=48.93)$, biology $(M=54.27)$ and psychology students $(M$ $=62.00)$ fell in-between. Simple comparisons with sidak adjustment revealed that ratings for business students were significantly lower than for all other groups, all $p$ s $<.05$, except for law students, $p=.50$.

\section{Participants and Design}

Seventy-five students from the University of Jena, Germany, participated in the data collection in a laboratory based on the campus of the university. Participants were students of different majors. After the experiment participants received money for their participation. The experiment was run on computers using Authorware software in individual cubicle spaces. The experiment consisted of a 2 (Information: no additional information, additional information) $\times 2$ (Category: business student, student of Romance languages) mixed design with the latter variable being manipulated within subjects. Additionally, I ran a control group in which participants did not get any feedback.

\section{Procedure}

After arriving in the laboratory participants were placed in closed cubicles so that they could not take notice of other people in the laboratory. Participants in the two experimental conditions were told that they would take part in a game through which they could earn money dependent on their own and the other player's decisions. They would earn points throughout the game which would be transferred to money at the end of the experiment. They were further informed that the experiment is run simultaneously in several laboratories around the campus and that they would be connected in each round of the game with another participant from one of the laboratories. The game consisted of a resource allocation task and went on for three rounds. In fact decisions were made by a preprogrammed computer and participants in each round got either the feedback of a fair or unfair decision of their "co-player".

At the beginning of each round participants received 12 points. In each round they were allegedly connected with another player via the server and they were presented with 
some information about the fictitious other player (e.g., the major). This was followed by a faked lottery to distribute the two roles in the game. In two of the three rounds participants got the role of Player A. Player A was not allowed to take any decisions. His/her 12 points were transferred to Player B and while transferring points were tripled. Participants were informed that Player B now has to take a decision how to distribute the 48 points (his/her 12 points plus 36 transferred points) between herself/himself and Player A. While waiting for the decision, Player A was asked how many points he/she expects to get back. Then, Player A got informed about Player B's decision. This feedback was followed by a series of questions. First of all, participants had to write down in an open-ended format how they explain to themselves the decision of Player B; next, they had to rate students with the same major like Player B in general; next, they indicated how much they were surprised by the feedback, and at the end of each round, participants could comment on the events.

During the first round, participants got the feedback to play against a student of the biology department. As pretests have shown, this seems to be a group about which people hold rather average expectations regarding warmth. In this first round, participants were placed in the role of Player A and received 24 points from Player B. This round has been included to reinforce fairness norms and to make participants familiar with the game. In round 2 participants were allegedly connected with a business student (target category), a group which is regarded as rather low on warmth. In one experimental condition participants just learned that the other player is aged 21 and studies business administration. In the other experimental condition, they additionally learned that he/she is specialized in marketing and production management. This fictitious Player B transferred 0 points back, which was supposed to be a supercongruent behaviour. In round 3 participants were assigned to the role of Player B. Hence, it was their turn to take a decision. This round was included to check whether participants will generalize the negative experience they just made in round 2 to all different kinds of categories. They were connected with a student of Romance languages, a category about which people might hold rather average expectations regarding warmth $^{22}$. First, they had to judge the group in general, with respect to the same attributes than in the rounds before. Then, participants took a decision about the back-transfer to

\footnotetext{
${ }^{22}$ This category was not been included in the pre-test. It was still assumed that students might not have any firm opinion about this category related to warmth. Results in the main study confirmed this assumption.
} 
Player A. They had to explain their decision. After the game had been finished, participants were carefully debriefed and got 3 Euros for compensation.

Participants in the control group went through the same procedure with the only difference that they should just imagine playing a game like this and they received no feedback from other players.

\section{Dependent measure}

Participants had to estimate in round 2 how many points they will receive from the other player who was told to be a business student. At the end of round 2, participants were asked to judge business students in general on five traits measuring warmth (trustworthy, fair, capable of teamwork, moral, warm). All ratings were given on scales ranging from 1 (not at all) to 7 (extremely). The same trait ratings followed in round 3 for students of Romance languages.

\section{$\underline{9.2 .3 \quad \text { Results }}$}

Three participants were excluded from the analysis because they were identified as outliers based on the criterion $z>2$ on one of the central dependent variables. One of them was outlying on the warmth and two on the expectancy rating for the business student. The first round just fulfilled the purpose of making participants familiar with the game. Results of this round will, hence, not be reported. Six traits capturing warmth were averaged for business students $(\alpha=.72)$, and for students of Romance languages $(\alpha=.90)$. All simple comparisons reported below were conducted with sidak adjustment.

\section{Expectancies}

An ANOVA with the dependent variable 'expected pay-back from business student' was conducted comparing 3 conditions (no additional information vs. additional information vs. control group) ${ }^{23}$. The Analysis yielded no significant effect, $F(2,69)=2.23, p=.12$ (see Table 7). Simple comparisons revealed that the difference between the expectation for the business student in the experimental condition without additional information and for the business student with additional information was not statistically reliable ( $M=9.88$ vs.

\footnotetext{
${ }^{23}$ Students of Romance languages are not included since participants were put in the role of Player B when connected with these students. Hence, there is no expectancy rating.
} 
$M=12.78), p=.40$, which supports the assumption that the chosen information was neutral.

Table 7. Means and standard deviations on expectancy values dependent on experimental condition (no exemplar, exemplar without additional information, exemplar plus additional information) (Study 7)

\begin{tabular}{lccc}
\hline Category & $\begin{array}{c}\text { No additional in- } \\
\text { formation }\end{array}$ & $\begin{array}{c}\text { Additional informa- } \\
\text { tion }\end{array}$ & Control \\
\hline Business & $9.88(7.68)$ & $12.27(7.24)$ & $8.48(5.79)$ \\
\hline
\end{tabular}

Note. $\mathrm{N}=72$. Numbers represent the expected payback from the business student.

\section{Expectancy violation}

In order to check whether the false feedback in round 2 differs from what people would have expected from a business student, I carried out a t-test comparing the expectancy value against 0 . The $t$-test revealed that the expectancy differed significantly from what participants received in the feedback, $t(72)=12.23, p<.05, d=2.04$. This finding indicates that the information in the feedback was indeed deviating from participants' stereotypical expectations in direction of the stereotype and thereby can be regarded as supercongruent.

\section{Warmth stereotypes}

In order to analyze the effect of the supercongruent feedback in the second round on the stereotype of the categories a 3 (no additional information vs. additional information vs. control group) x 2 (Business, Romance) ANOVA was carried out with the latter factor being varied within subjects. A significant main effect for category occurred, $F(1,69)=$ $170.51, p<.05, \eta^{2}=.71$. Business students were judged as less warm than students of Romance languages $(M=2.78$ vs. $M=4.85$ ). Additionally, the expected interaction with condition rendered significant, $F(2,69)=3.27, p<.05, \eta^{2}=.09$ (see Table 8 ).

In order to understand the interaction, two ANOVAs were carried out for each stereotype separately. While the analysis was marginally significant for the category of business students, $F(2,69)=2.82, p=.067, \eta^{2}=.08$, no effect occurred for the other stereotype, $F(2,69)=1.47, p=.238$. Finally, the specific hypothesis was tested, namely that in the no-additional-information experimental condition business students will be 
judged as less warm than in the other two conditions ( $\mathrm{C} 1$ : -211 ). No difference should occur between the additional information experimental condition and the control condition (C2: 0 - 1 1). The first contrast yielded a significant effect, $F(1,69)=5.52, p<.05, \eta^{2}=.07$, whereas the second contrast did not, $F<1$. Participants perceived business students as less warm after they received the negative feedback and had no additional information about the player $(M=2.45)$, but they did not change their stereotype when they additionally learned about the other player's specialization $(M=2.90)$ compared with a control condition $(M=$ 2.99).

Table 8. Means and standard deviations on warmth ratings for different categories dependent on experimental condition (exemplar without additional information, exemplar plus additional information, no exemplar) (Study 7)

\begin{tabular}{lccc}
\hline Category & $\begin{array}{c}\text { No additional in- } \\
\text { formation }\end{array}$ & $\begin{array}{c}\text { Additional informa- } \\
\text { tion }\end{array}$ & Control \\
\hline Business & $2.45(.92)$ & $2.92(.62)$ & $2.99(.98)$ \\
Romance languages & $4.83(1.39)$ & $4.36(.72)$ & $4.85(.99)$ \\
\hline
\end{tabular}

Note. $\mathrm{N}=72$. Means presented are rated on a 1-7 scale with higher numbers indicating more warmth..

Surprise

Participants in the control group did not get any feedback, therefore they were not asked to indicate their level of surprise. The surprise item was subjected to a t-test to compare the two experimental conditions (no additional information vs. additional information). The analysis did not provide evidence for a significant difference between the condition without additional information $(M=3.04)$ and the condition with additional information $(M=3.96), t(47)=1.38, p=.17$. Mean pattern reveals that participants were somehow less surprised by the supercongruent business student when they received no additional information.

\subsection{Discussion of Part III}

The main purpose of this study was to test whether assimilation of the stereotype to a supercongruent exemplar can be blocked by providing additional, neutral information about the exemplar. More precisely, it has been tested whether a) the stereotype of business 
students as lacking warmth would be polarized when confronted with an unfair and selfish business student, and b) whether this polarization can be blocked by additional information about the specialization of the student (i.e., neutral information). The results first of all support the findings of part I and part II. People seem to be prone to assimilate their stereotype to supercongruent exemplars which implies that stereotypes easily get polarized. After participants received the information that a business student has treated them in an unfair manner, they rated business students as less warm compared with a control condition. More importantly, results of this study show that the polarization does not seem to be inevitable. The additional, pseudo-relevant information that the business student is specialized in marketing and production management blocked the generalization process. Participants in this condition judged business students in total as equally warm as the control condition.

Several accounts have been mentioned by Kunda and Oleson (1995) to explain the exclusion of an incongruent exemplar due to additional information. Not all of these accounts seem plausible to explain the present finding of an exclusion of a supercongruent exemplar as will be outlined below.

\section{Subtyping triggered by surprise}

Kunda and Oleson (1995) reasoned that people use the neutral information to explain away the surprise they experience through incongruent exemplars. They further argued that supercongruent exemplars will lead to generalization no matter if neutral information is present or not since they assumed this kind of information not to come as a surprise. The present study provided support for their assumption that people are not very surprised by a supercongruent exemplar. Mean levels of surprise were below the mid-point of the scale ( $M=3.47$ vs. 4 ). These rather low levels of surprise are in line with the findings of previous studies within this dissertation which demonstrated that supercongruent exemplars are perceived as more typical than incongruent exemplars. It is also in line with the reasoning that supercongruent deviance can be best explained in terms of a quantitative deviance - prototype and exemplar differ in terms of extremity -, while incongruent deviance is likely to imply more of a qualitative deviance - prototype and exemplar do not possess the same trait. However, although people were not very surprised by supercongruent information, because it seems to be in line with their stereotype, additional, pseudo-relevant information still blocked generalization. Surprise might certainly be a reasons for subtyping in 
case of incongruent information, but the exclusion of supercongruent exemplars cannot be explained by this feeling.

Subtyping triggered by motivation to maintain the stereotype

Kunda and Oleson (1995) reasoned that people use the additional information in order to explain away the incongruent deviance and thereby protect their stereotype. Assimilating the stereotype towards supercongruent exemplars does not imply to give up or weaken the stereotype. Instead, it becomes even stronger and differences between groups become accentuated. Hence, people should not feel the urge to explain away the deviance in order to protect their stereotype.

\section{Subtyping due to reduced feature-based similarity}

Besides surprise and motivation to maintain, two alternative explanations for the effect have been mentioned by Kunda \& Oleson (1995): One account is based on Tversky's model of similarity (1977). Neutral information that is not included in the representation of the stereotype decreases the similarity between the deviant exemplar and the stereotype. If the number of attributes increases that is unique to either of them, the exemplar should appear less typical of the stereotype. This should result in reduced generalization. Analogously, it has been shown that the impact of stereotypes on the perception of individuals is reduced by neutral attributes (the dilution effect, see Nisbett, Zukier, \& Lemley, 1981). However, the additional, neutral attributes chosen for the present study - specialization within the subject - is not really unique to the exemplar. A large amount of members of the category might possess this attribute. Furthermore, no evidence for a dilution effect occurred. If similarity between the exemplar and the stereotype would have been considerably reduced due to the additional information, participants in the present study should have expected a higher pay-back in this condition compared with the experimental condition without additional information. Although there was a slight tendency to expect more in the additional-information condition, this tendency was not significant. Peters and Rothbart (2000) have demonstrated that not all information that is neutral to the outcome of predictions is able to create a dilution effect. In their studies dilution just occurred if the additional information influenced the interpretation of the categorical information and rendered the individual less typical for the group. It is rather unlikely to assume that the information 
about the specialization in marketing and production management should appear untypical for business students in general. Nevertheless, I checked this possibility in a post-hoc test by asking 15 participants to rate the goodness of fit of this information with their image of business students $(1=$ very strongly disagrees with my image of a typical business student to $7=$ very strongly agrees with my image of a typical business student, with $4=$ unrelated to my image of a typical business student). A mean value of $M=5.33, S D=1.23$, which significantly deviated from the scale-midpoint $4, p<.05$, indicates that participants did not find this additional information untypical for business students in general; to the contrary, they perceived it as rather typical. Based on these results and arguments, I find it rather implausible to assume that the generalization was blocked because of reduced similarity between the stereotype and the deviant.

\section{Attenuated generalization due to an increase in perceived variability}

Another account draws back to the finding that people generalize less from exemplar to category when the category is believed to be variable (Nisbett, Krantz, Jepson, \& Kunda, 1983). The additional information could have made participants aware of the possibility that the category can vary on additional dimensions. The additional information that was provided in the present study shares some similarities with subgrouping manipulations. Subgrouping instructions encourage subjects to make discriminations within the context of an activated category (Rothbart \& Park, 2004). A typical example of a subgrouping instruction would be to classify a list of occupations, all engaged in by women, into subcategories. To realize that business students can specialize in different subdisciplines within their field has probably increased the perceived variability, like subgrouping instructions also increase the perceived variability (Park, Ryan, \& Judd, 1992). Some researchers have argued that people generalize less from group members to their groups when the group is perceived to be variable (Nisbett et al., 1983; Quattrone \& Jones, 1980). Yet, other researchers have demonstrated that the manipulation of variability interacts with pattern of disconfirming information in the sense that stereotypes became attenuated even due to concentrated disconfirmers when the group was believed to be variable. Concentrated disconfirmers had no impact when the group was perceived to be less variable (Hewstone \& Hamberger, 2000). The relation between perceived variability and the impact of deviant exemplars has not been clearly understood yet. The present results imply the possibility that an increased vari- 
ability through drawing attention to the subgroup level could buffer a stereotype against getting polarized due to supercongruent exemplars. Future research should test this assumption more directly by 1) manipulating a group's perceived variability and testing in which way this manipulation affects the generalization of a supercongruent exemplar and 2) providing evidence that additional, neutral information actually increases perceived variability. Because of the diverging effects of super- and incongruent information (one strengthens the stereotype and one attenuates it), it would be also interesting to cross pattern of a variability manipulation with the two directions of deviance. Perceived variability might impact generalization of a supercongruent and an incongruent exemplar differently since one leads to a more stereotypic view and one to a less stereotypic view of the category.

Futures studies, additionally, should test, whether a generalization occurs on the sub-group level. Participants in the present study have not been asked to indicate their belief regarding business students specialized in marketing and production management. Hence, we do not know, whether the supercongruent information got associated with the additional information.

The present paradigm constitutes a promising way of studying stereotype change because it is more unobtrusive than the previously used paradigms. But it still can be improved. The rules of the game, for example, were quite complicated and some participants might have not understood them entirely. The game might be simplified by leaving out the first transfer and triplication of the points. Instead, one of the players, drawn by lot, receives all the points and is asked to distribute them among him-/herself and the other player (dictator game).

While in direct contact we certainly almost always receive additional information about a member of a group, indirect contact via the media (e.g., in newspaper announcements) many times just provides us with the misdeeds and the group affiliation (e.g., nationality) of someone. The present results provide first evidence that it might be of great value to find out more about the possibilities how stereotypes can be buffered against the impact of supercongruent information. Although we still have to learn more about the process and whether all kinds of additional information may have this buffering effect, it is clearly a positive news to state that polarization of stereotypes is not inevitable. 


\section{General Discussion}

\subsection{Overview}

The research question of the present dissertation developed upon the observation that stereotypes might be more likely to get polarized than attenuated. I was basically interested in the perception of one-single supercongruent exemplar and its impact on the central tendency of a stereotype. In order to explore the processes involved in stereotype maintenance and change, I was specifically aiming at (1) comparing the perception and impact of one single supercongruent exemplar to that of one single incongruent exemplar with the same objective amount of deviance from the prototype. Assuming that supercongruent information is likely to be generalized to the group as a whole, I additionally wanted to explore (2) in which kind of situations this generalization does not occur. Learning about these circumstances should help to (a) clarify the underlying mechanism to the basic effect and (b) develop strategies how an assimilation of the stereotype to supercongruent information can be circumvented whenever its unwanted (i.e., stereotypes might be protected from getting polarized).

Two lines of research stimulated the development of the present hypotheses: research on stereotype change and models based on the accentuation principle, such as the accentuation theory and self-categorization theory (SCT). The first hypothesis is based on the meta-contrast-ratio (Turner \& Oakes, 1989; cf. chapter 5) which states that the typicality of category members depends upon an intra- and intergroup comparison. Incongruent exemplars have been previously defined as deviating in the direction of a comparison category (Brewer et al., 1981), whereas supercongruent exemplars were defined now as deviating in the opposite direction (cf. chapter 3). Based on this notion and the meta-contrast principle the first hypothesizes states: Supercongruent exemplars should be perceived as more typical than incongruent exemplars.

Goodness-of-fit (or perceived typicality) of an exemplar has been shown before to be one of the main determinants of generalization from the exemplar to the group as a whole (e.g., Johnston \& Hewstone, 1992; Kunda \& Oleson, 1997; Rothbart \& John, 1985; Rothbart \& Lewis, 1988; Wilder, 1984; cf. chapter 4). Based on this finding and the first hypothesis concerning perceived typicality, the second hypothesis states: One supercongruent exemplar should exert a stronger influence on the categories' stereotype than one incongruent exemplar. Since assimilation of the prototype to supercongruent exemplars increases the difference between groups (target and comparison cate- 
gories), whereas assimilation to incongruent exemplars decreases the difference, this assumption is also in line with accentuation theory which states that people strive to maximize differences between groups (e.g., Krueger, 1991; 1992; Krueger \& Rothbart, 1990; Krueger et al., 1989; Tajfel \& Wilkes, 1963; cf. chapter 4). The first two hypotheses were tested in the studies summarized in Part I.

Study 1 examined the perception of congruent and deviating exemplars and provided evidence that supercongruent exemplars can be distinguished from congruent ones. Participants did differentiate between a congruent and a supercongruent exemplar, especially when asked about the perceived deviance of the exemplar from the stereotype. The supercongruent exemplar was perceived as more deviating than the congruent one and as equally deviating than the incongruent one. This finding constitutes a relevant precondition for the present research because it confirms that deviance does exist in two versions: in direction of the stereotype (supercongruent) and in direction of a contrast category (incongruent). However, when it comes to perceived typicality, the two deviating exemplars were judged differently. The supercongruent exemplar was perceived as more typical than the incongruent one. Hence, the same objective deviance seems to translate to different degrees of subjective deviance.

This finding of higher perceived typicality in case of supercongruent exemplars compared with incongruent ones, supports the first hypothesis and was replicated by findings in Study 3, 4, and 5. ${ }^{24}$ Study 4 demonstrated that even the same exemplar is perceived differently depending on the judgmental context. It thereby excludes the alternative hypothesis that differences in perception of the two kinds of deviating exemplars are just due to differences in their extremity. When the contrast category rendered the exemplar supercongruent by being situated on the opposite side of the continuum, the exemplar was perceived as more typical than in those conditions when the context rendered the exemplar incongruent by lying on the same side of the continuum. This effect occurred although the stereotype was learned with both contrast categories being salient. Study 5 once more replicated the difference in perceived typicality between the incongruent and supercongruent exemplar. In this study, however, the manipulation check indicated that the difference between the incongruent and congruent exemplar was bigger than between the congruent and supercongruent exemplar. A post-hoc test, in which the same exemplars were presented without information about group-

\footnotetext{
${ }^{24}$ The other studies either did not measure perceived typicality (Study 2), did not include an incongruent condition (Study 6,7 ), or/and used typicality items as a focus manipulation and hence they differed between conditions (Study 6).
} 
membership, revealed equal distances. This finding is inconsistent with Study 4, in which exemplar ratings were not influenced by the kind of comparison category. Because the experimental paradigms of Study 4 and 5 differed in various respects, I cannot draw firm conclusions about the cause for this differential effect. One reason might be, that Study 5 employed a pre-existing stereotype, whereas Study 4 used a stereotypelearning paradigm and, furthermore, the target group was believed to be average on the target dimension compared with the other categories. The expectations in Study 4 might have been less firm than in Study 5 and this could be one reason why the exemplar judgment was not influenced by the overall prototype.

The second hypothesis, which states that one supercongruent exemplar should exert a stronger influence on a category's stereotype than one incongruent exemplar, was supported by findings of Study 2, 3, 4, and 5. ${ }^{25}$ Study 2 showed that perceived inter-group differences increased when confronted with a supercongruent exemplar, whereas they remained constant when participants were confronted with an incongruent exemplar. In Study 3 a paradigm was used that is more common to research on stereotype change. Compared with a baseline, the prototype was polarized after learning about a supercongruent exemplar. No changed occurred due to an incongruent exemplar. In Study 4 the same exemplar led to stronger assimilation tendencies when the context rendered it supercongruent than when the context rendered it incongruent. Different from all the other studies within this dissertation, assimilation occurred in Study 4 in all conditions, in the incongruent ones as well. Two explanations seem plausible to account for this finding. Either it is due to the greater sensitivity to detect change in this study based on the repeated measurement design, or, this effect occurred because the study did not employ a pre-existing stereotype. As mentioned before, the strength of the artificial stereotype, which developed during phase 1, might have been lower compared with the one of pre-existing stereotypes, and hence, participants used any additional kind of information about the category. In Study 5 the prototype was assimilated to the supercongruent exemplar when the stereotype measurement directly followed the exemplar presentation, whereas no change could be detected in case of an incongruent exemplar.

In sum, Part I provided evidence that people perceive a supercongruent exemplar as more typical than an incongruent exemplar and that the first one has a larger impact on a category's stereotype than the latter one. The same results were demonstrated for stereotypes with negative as well as positive valence. Part II and Part III of the present

${ }^{25}$ Study 1 did not comprise a stereotype measure; Study 6 and 7 did not include a comparison between an incongruent and supercongruent conditions. 
dissertation dealt with the question whether this stereotype polarization is inevitable. Or, to put it differently: What are the circumstances in which generalization from a supercongruent exemplar to the category is less likely to occur?

It has been assumed that incongruent exemplars are likely to pose a qualitative deviance to the prototype because the exemplar is likely to not possess the stereotypic attribute. Therefore, the deviance might be easily recognized by perceivers and lead them to engage in a process of dissimilarity testing. Supercongruent exemplars, on the contrary, might trigger more similarity testing because they are perceived as qualitatively similar to the category. Their deviance is just a matter of degree and might be easily overlooked. Confronted with this kind of exemplar typicality or deviance might not be judged automatically. Directing participants' focus towards the supercongruent deviance should render generalization of the exemplar to the category less likely to occur. Letting participants rate the typicality of these exemplars could fulfil this purpose. Technically speaking, order of typicality and stereotype assessment should have a bigger impact for supercongruent exemplars than for incongruent ones.

Study 5 demonstrated that asking participants to judge the deviance and typicality of a supercongruent exemplar before the stereotype was assessed, indeed prevented them from generalizing to the category. Incongruent exemplars did not influence the prototype no matter which order of measurement. This finding speaks to the fact that the deviance of an incongruent exemplar is detected more automatically. The goal of Study 6 was to test the processes involved in generalization from supercongruent exemplars more directly. Firstly, it aimed at replicating the findings obtained in Study 5, and secondly, focus of comparison (similarity vs. dissimilarity) was manipulated more directly by posing different kinds of typicality questions. Parallel results occurred in the condition in which the stereotype was assessed directly after the manipulation, and in the condition in which participants were induced to focus on similarities. In both conditions, an assimilation to the supercongruent exemplar occurred. Participants who were asked to judge the deviance of the exemplar from the prototype (dissimilarity focus) exhibited no generalization on some of the stereotypic traits and showed a slight contrast effect on others. In general, findings of Study 6 speak to the fact that a focus on similarities is the default process people engage in when confronted with a supercongruent exemplar.

The finding that stereotype assimilation to supercongruent exemplars does not occur once people process the new information with a focus on differences, is informa- 
tive about the mechanisms involved in stereotype polarization. Part III summarizes one study that also demonstrates circumstances in which polarization does not occur, but this time the basic aim was to develop strategies how stereotype polarization can be circumvented. Previous research has shown that additional, neutral information about incongruent exemplars is used to justify subtyping and, hence, makes generalization less likely to occur (Kunda \& Oleson, 1995, Yzerbyt et al., 1999). In contradiction to previous research (Kunda \& Oleson, 1995; Exp. 4), Study 7 demonstrates that additional, neutral information also decreases generalization from a supercongruent exemplar. Participants in Study 7 were confronted within a game with a very egoistic co-player who was told to be a business student (supercongruent information). This experience made them rate business students in total as less warm compared with a control group which did not receive any exemplar information. Hence, participants assimilated their prototype to the supercongruent exemplar. This assimilation did not occur when participants received the additional information that their co-player was specialized in marketing and production management. This effect might have occurred due to an increase in perceived variability. Previous research dealing with the effect of incongruent exemplars has demonstrated that people seem to generalize less when they perceive the group to be very heterogeneous (Nisbett et al., 1983; Quattrone \& Jones, 1980). Unfortunately, this interpretation can not be tested with the present data. Alternative accounts, however, do not seem to fit some of the results of Study 7 (cf. chapter 9.3).

In sum, I presented evidence that supercongruent and incongruent exemplars are perceived differently concerning typicality and lead to different consequences for the stereotype. Assimilation of the stereotype seems to be the default process when facing supercongruent information. Concerning incongruent information, no impact was observed in the present studies with one exception: The stereotype of an artificial category was also assimilated to incongruent exemplars as well.

\subsection{Underlying Processes}

The present research found convincing support for the assumed asymmetry in the processing of information that mismatches the stereotype. Incongruent information did by and large not influence the judgment of the group as a whole, whereas the default reaction when dealing with supercongruent information was a generalization to the stereotype, which is why stereotypes easily get polarized. In contradiction to the hypotheses, the present data does not support the idea that this asymmetry is caused by 
differences in perceived typicality since no mediation occurred in Study 4. The relation between the assumed mediator (perceived typicality) and the outcome (assimilation), was not quite strong, and as data suggests it might not be linear. As argued in the discussion of Part I, this result might be due to a paradox which has been previously mentioned by Rothbart and colleagues: "Disconfirming information associated with exemplars may have two contradictory effects on stereotype change. First, as the disconfirming nature of the information increases, the potential to modify the stereotype also increases. Second, as the magnitude of disconfirmation increases, the associated exemplar decreases in its goodness-of-fit to the stereotype" (Rothbart, Sriram, \& Davis-Stitt, 1996, p. 311). Goodness-of-fit has been shown to be one of the key determinants whether to include or not an exemplar into the representation of a category. Perceived typicality might be more likely to mediate stereotype change, when typicality varies as a function of additional attributes compared with when it varies as a function of extremity of deviance or direction of deviance. To make this more obvious: Those people who hold a rather extreme stereotype might perceive a supercongruent exemplar as highly typical because it might not deviate from their expectations at all. ${ }^{26}$ But since they do not receive deviating information, there is no reason for them to change their stereotype. The relationship between perceived typicality and stereotype change might be by far more complicated as sometimes assumed. Previous research, which demonstrated a bidirectional link between perceived typicality and stereotype change, suggested to regard perceived typicality also as an outcome of categorization (Richards \& Hewstone, 2001). Furthermore, the finding that the stereotype judgment as well as the typicality judgment was dependent on the order of measurement between the two constructs (Study 5), also speaks to the fact that their relation might be quite complex which makes it difficult to detect a mediation even if it exists. Due to the order effect, typicality can only be assessed after the stereotype judgment otherwise it prevents the effect from occurring. Yet, the mediator ideally should be measured before the outcome variable (Kenny et al., 1998, p. 262). Due to this reasons, it might be a more promising way to test a moderation hypothesis in order to investigate the underlying process of the present effect (e.g., Spencer et al., 2005).

Theories dealing with accentuation, thus with the tendency to increase differences between groups (SCT and accentuation theory), have rarely specified the process.

\footnotetext{
${ }^{26}$ Remember that supercongruent exemplars in the present studies were not created for each participant individually based on their pre-values. Hence, an exemplar which is on average supercongruent might be congruent for some of the participants (for those who's stereotype was extreme from the beginning).
} 
Three plausible mechanisms, namely biased encoding, biased retrieval and differential or biased integration of the information, have been suggested to explain the accentuation principle (Krueger et al., 1989; Krueger \& Rothbart, 1990). Biased encoding operates at the stage of exemplar perception, biased retrieval refers to memory processes, and biased integration operates at the stage of information usage in forming an overall judgment. As outlined below, biased encoding and retrieval seem rather unlikely to explain the present findings. In fact, results rather suggest that the provided information might have been integrated in a biased manner.

Biased encoding refers to distortions in the perception of individual stimuli which in turn lead to a distorted perception of the category. By providing participants with numerical information I tried to decrease ambiguity of the exemplar information. Still, participants had to interpret the test results and translate them into trait judgments. Thus, exemplar judgments might have been biased. However, the present data suggests that this was not the case. First of all, incongruent and supercongruent exemplars were perceived as equally deviating (Study 1). Secondly, range and standard deviation in the exemplar judgment were not bigger for the incongruent exemplar than for the supercongruent one (Study 3). Third, although Study 4 used the same exemplar and manipulated direction of deviance by means of the comparison category, still greater assimilation was obtained in case of supercongruent information. Fourth, the exemplar judgment in Study 4 was not affected by type of comparison category. And fifth, exemplar judgments in Study 5 seem to be affected by the group-membership, but in a way that made supercongruent exemplars less deviating than incongruent exemplars.

Summarized by the term biased retrieval are several mechanism dealing with impaired memory. Firstly, information that confirms expectations has been demonstrated to be better remembered than information that is contradicting (e.g., Rothbart, Evans, \& Fulero, 1979). Secondly, difference-enhancing stimuli might be recalled better and their frequency might be overestimated due to their greater extremity and thereby salience (Tversky \& Kahnemann, 1973). Thirdly, difference-attenuating stimuli might be erroneously recalled as contextual stimuli. Krueger and Rothbart (1990) showed that category-enhancing information was remembered better than category-attenuating information. Participants in their studies were confronted with a whole list of exemplars. Since participants in the present studies were just presented with one exemplar and were not distracted by some other task it seems rather unlikely that they might have "forgot- 
ten" about the incongruent information. Hence, it seems rather unlikely that memory processes could have driven the effect.

Among the afore mentioned three plausible mechanisms, biased integration seems most promising. Biased integration takes place at the time of forming an overall judgment. But why is it that incongruent and supercongruent information is used differently in forming a judgment? One potential explanation for the differential impact of supercongruent and incongruent information is provided by social judgment models, like the selective accessibility model (SAM; Mussweiler, 2001; 2003; Mussweiler \& Strack, 2000). SAM explains when a judgment is likely to be assimilated to and when it is likely to be contrasted away from the present context. Assimilation should occur if judges engage in a process of similarity-testing, and contrast results if they engage in dissimilarity testing. Both kinds of hypotheses will render different kinds of knowledge about the judgmental target accessible. Results of Study 5 and 6 suggest that generalization of supercongruent information to the category was affected by an intermediate focus manipulation. Assimilation of the stereotype to the supercongruent exemplar did not occur when participants had to judge the deviance of the exemplar from the category inbetween. This kind of judgment might have served the function of directing participants' focus to the deviance and triggering a dissimilarity focus. The fact that the same results, namely generalization, were obtained when participants had to judge the stereotype directly and when they had to judge perceived similarity in-between, indicates that the default process when confronted with a supercongruent exemplar is to focus on similarities. The processing of an incongruent exemplar, on the contrary, does not seem to be affected by a deviance judgment. This suggests that their deviance is acknowledged more automatically triggering some sort of dissimilarity testing. Yet, it remains to be tested whether focus of comparison indeed mediates the impact of direction of deviance on stereotype change. Do supercongruent exemplars actually lead to a focus on similarities between exemplar and category? And, do incongruent exemplars indeed lead to a focus on differences? Further research might test these processes more directly for example by measuring the focus of comparison in an unrelated task after participants had been confronted with a supercongruent or incongruent exemplar. According to the procedural priming logic a certain focus of comparison dominating in a certain situation carries over to a subsequent comparison, even if they are unrelated (see Mussweiler et al., 2003). 
According to SAM (Mussweiler \& Strack, 2000), assimilation is the outcome of biased search for similar evidence. The model, hence, focuses on knowledge activation. In the present context, that would imply that a supercongruent exemplar leads to a memory search for similar exemplars. The inclusion/exclusion model, then again, regards assimilation as the outcome of an inclusion of the contextual stimuli into the representation of the judgmental target (Schwarz \& Bless, 1992). This model, hence, focuses on knowledge use. Accordingly, a supercongruent exemplar does not necessarily influence the retrieval of stored exemplars, but leads to an assimilation of the stereotype by its addition to other chronically accessible exemplars. It seems rather implausible that just the first process, suggested by the SAM model, should be involved in the assimilation of the stereotype to supercongruent exemplars, without the second one, suggested by the inclusion/exclusion model. However, it remains to be tested whether supercongruent exemplars influence the set of retrieved exemplars, and additionally, whether supercongruent exemplars happen to change abstract knowledge. One first step to investigate these questions might be to test the durability of the observed assimilation of the stereotype. Whether or not the stereotype is still polarized some time after the manipulation in a different context, might tell us something about the nature of the effect. If stereotypes go back to the initial level as soon as the supercongruent exemplar is not salient anymore, then abstractions might have remained unchanged. To my knowledge, no study has so far tested the duration of experimentally induced stereotype change. In order to circumvent ethical concerns and avoid additional experience with the category in-between, this could possibly be tested by employing an artificial stereotype.

But why is it that supercongruent information is processed with a focus on similarities, whereas incongruent information is processed with a focus on dissimilarities? The selective accessibility model assumes that whether a judge engages in a process of similarity or dissimilarity testing depends on several moderators. One of those is psychological closeness (Mussweiler, 2003). If target and standard are perceived as close, assimilation is more likely to occur, whereas contrast appears when they are not. It has been argued within the present dissertation that the asymmetry in the processing of the two kinds of deviating information might be due to the different nature of their deviance: Incongruent exemplars are likely to pose a qualitative deviance, whereas supercongruent exemplars are likely to constitute a quantitative deviance. This difference might even hold for Study 4, where the same exemplar was rendered super- or incon- 
gruent by different comparison categories. But in this case the difference is probably due to the fact that the target category is represented differently according to the context: Although the target category was judged as average in both conditions, it might be still represented as "the higher category" in context of a low comparison category, and as "the lower category" in context of a high comparison category. Unfortunately, the present data does not allow to test this assumption.

The present differentiation between a quantitative and a qualitative difference shares some similarities with the notion of a psychological mid-point within research on persuasion (Fishbein \& Lange, 1990; Lange \& Fishbein, 1983; Nemeth \& Endicott, 1976). This research has shown that persuasion is less successful if the position of the source and the receiver of a persuasive communication fall on different sides of a response dimension then if the positions fall on the same side. A midpoint was characterized as the "the marker that identifies different substantive categories" (Fishbein \& Lange, 1990, p. 189) and that allows the receiver to "categorize the communicator's position into 'same side' or 'opposite side' of the issue" (Nemeth \& Endicott, 1976; p. 12). Comparably, the psychological midpoint on the dimensions used to present exemplar information in the present context might be the point that divides values into those that indicate possession of the stereotypic attribute and those indicating that someone does not possess the attribute, or is even characterized by its antonym (e.g., the IQ-value that constitutes the border between intelligent and non-intelligent). It has been argued and demonstrated before that receivers of a persuasive communication change their initial attitude more when confronted with a 'same side'-persuasive position than when confronted with an 'opposite side' message (Nemeth \& Endicott, 1976). This difference in persuasive power especially occurred when the qualitative difference was made salient. Within most of the present studies, participants had to judge the exemplar on the stereotypic dimension first. ${ }^{27}$ While thinking about whether the exemplar possesses the stereotypic attribute or not the qualitative difference might have become apparent. It remains to be tested whether the same asymmetry in the processing of super- and incongruent information appears when no exemplar judgment precedes.

The present research constitutes a first test of accentuation principles in the context of stereotype change due to one additional exemplar. While researchers within social psychology have focused on explaining accentuation principles with motivational

\footnotetext{
${ }^{27}$ An exception was Study 2. But in this study the "psychological mid-point" might have been quite obvious because the IQ-value of 100 was mentioned in the introduction as the general average. Prototype and supercongruent exemplar were above 100, whereas the incongruent exemplar was below 100 .
} 
forces (e.g., Mullen, Brown, \& Smith, 1992), other researchers have argued for cognitive mechanisms (e.g., Goldstone, 1994; Krueger, et al., 1989; Krueger \& Rothbart, 1990). Results should be regarded as a first step in answering the process question. They suggest that the process might not be driven purely by a motivation to protect and strengthen the stereotype. Instead, they point to a rather cognitive process. Further studies are needed to understand the involved mechanisms in dept. These studies might include measures or manipulations of focus of comparison and markers of category activation (e.g., repetition priming; Quinn \& Macrae, 2005), Additionally, it might be worthwhile to include measures of subtyping beyond perceived typicality (see Park et al., 2001).

\subsection{Results in Light of Previous Findings and Theories}

The present dissertation builds upon diverse lines of research which are all explaining in one way or another the phenomenon of categorization. The "stereotype change"-paradigm has focused on change due to incongruent exemplars of the target group. Most of the studies have shown support for the subtyping model of stereotypes (e.g., Johnston \& Hewstone, 1992; Johnston et al., 1994; Weber \& Crocker, 1983; see Hewstone, 1994, for a review), which is in fact a model of non-change because it assumes that incongruent exemplars lead to the creation of subtypes, which are regarded as exceptions and, hence, leave the stereotype unchanged.

The present studies which were conducted in order to compare the impact of supercongruent and incongruent exemplars, replicate previous research in showing that incongruent exemplars hardly affect the central tendency aspect of a stereotype. This general lack of influence has often been thought to be a consequence of the humble fit between exemplar and category as a whole (e.g., Johnston \& Hewstone, 1992; Kunda \& Oleson, 1997; Rothbart \& John, 1985; Rothbart \& Lewis, 1988; Wilder, 1984). Incongruent exemplars were perceived in the present studies as less typical than congruent or supercongruent exemplars, but the data does not support the idea of a mediation by perceived typicality (see 10.2). Consistent with previous research, Study 5 showed a tendency that incongruent exemplars lead to a less homogenous picture of the group (Garcia-Marques \& Mackie, 1999; Paolini et al., 2004). The lack of impact of incongruent information on the stereotype obtained ion the present studies is inconsistent with the inclusion/exclusion model (Schwarz \& Bless, 1992; 2007), which regards assimilation as the default process when dealing with exemplar information. Interestingly, studies 
supporting the inclusion/exclusion model often employ affective outcomes (e.g. favorability; Bless \& Schwarz, 1998; Bless \& Wänke, 2000; Bodenhausen et al., 1995). By conducting a meta-analysis Tropp and Pettigrew (2005) provided evidence that affective dimensions of inter-group attitudes are more likely to change due to contact experience than cognitive dimensions. Other studies that did include a stereotype judgment and support the inclusion/exclusion model did not comprise a control group without exemplar information (Bless et al., 2001). Hence, it is difficult to tell whether just assimilation, or contrast, or both occurred. Due to these reasons the comparability of the present results regarding incongruent exemplars and the ones obtained within research on the inclusion/exclusion model might be limited.

Deviating exemplars, however, do not always lack influence on the stereotype. A host of factors, like for example the extremity of deviance, the cognitive resources or the availability of neutral information to legitimate subtyping, have been shown to moderate the emergence of generalization or subtyping (e.g., Kunda \& Oleson, 1995; 1997; Yzerbyt et al., 1999). The present research expands the list by adding direction of deviance. It has been demonstrated that supercongruent exemplars are more likely to generalize to the group as a whole than incongruent exemplars. This asymmetry in likelihood for generalization was paralleled by an asymmetry in perceived typicality which was higher for supercongruent exemplars than for incongruent ones. These findings are in line with accentuation theory and the meta-contrast-principle put forth by the selfcategorization theory.

The self-categorization theory and the accentuation theory were built up on a classic experiment by Tajfel and Wilkes (1963) in which they demonstrated that perceivers who had to estimate the length of lines tended to overestimate the difference between lines that belonged to different categories. Although the accentuation effect has seldomly been replicated with the original paradigm (see McGarty, 1999), similar effects were demonstrated for attitude statements (Eiser, 1971; Eiser \& Stroebe, 1972; Eiser \& van der Pligt, 1982; McGarty \& Penny, 1988), trait valences (Krueger \& Rothbart, 1990), body weights (Krueger et al., 1989), perception of colors (Goldstone, 1995), or judgments of multi-faceted stimuli (Corneille \& Judd, 1999; Ford \& Stangor, 1992; Goldstone, 1994; 1996; Goldstone, Steyvers, \& Rogosky, 2003). A variety of different measures has been used to demonstrate the accentuation effect: 1) categorization accuracy for stimuli that deviate from the category's average (Goldstone, 1996), 2) estimated values for specific stimuli (Eiser, 1971; Krueger \& Clement, 1994), 3) proto- 
type judgments (Krueger et al., 1989; Krueger \& Rothbart, 1990), or 4) judgments of stimulus typicality (Corneille \& Judd, 1999). The present studies are demonstrating accentuation tendencies by use of the latter two measures: perceived typicality and central tendency judgment.

The results regarding perceived typicality are convincingly supporting metacontrast-principles developed within the SCT (Turner \& Oakes, 1989; cf. chapter 5). They replicate previous research which has demonstrated the meta-contrast-principle in the context of a salient ingroup-outgroup-differentiation. Abrams and colleagues have shown that members who deviate in their opinion towards an opposing group, called "anti-norm deviants", are perceived as more atypical than those who deviate away from the opposing group, called "pro-norm deviants" (e.g., Abrams et al., 2000). To my knowledge the present studies constitute the first attempt to apply meta-contrastprinciples to the context of social stereotypes without an ingroup-outgroup differentiation being salient.

The finding that contrast categories determine typicality has previously also been shown in the context of artificial category learning (Corneille \& Judd, 1999; Goldstone, 1996; Palmeri \& Nosofsky, 2001). By using portraits and applying morphing techniques, it has been demonstrated that caricatures are more easily categorized than centroids if the concepts' representation is dependent on some other concept (Goldstone, 1996; Nosofsky, 1991; Palmeri \& Nosofsky, 2001). Caricatures were thereby defined as those stimuli that depart from the central tendency of the category in the opposite direction of the central tendency of other, simultaneously acquired concepts. This definition sounds very much like the definition of supercongruent exemplars, but there is one important difference: Within research on caricatures 'central tendency' refers to the genuine average of the category which can be easily defined when dealing with morphed pictures. In the context of social stereotypes, however, 'central tendency' usually refers to the belief people hold about the average group member. Compared with the real average group member, this belief might already be distorted in direction of a caricature. Hence, the present results and these previous findings are not dealing with the same question, but both are a demonstration of the fact that typicality depends not just on within-group similarity. Especially Study 4 indicates that perceived typicality is dependent on the salient frame of reference. This finding is especially challenging for pure-prototype models which assume categories to be represented by one fixed prototype (see also Medin, 1989). It is more in line with the context sensitivity claimed by 
exemplar-based models (Smith \& Medin, 1981; Smith \& Zárate, 1992) and the selfcategorization theory (Oakes et al., 1994; Turner et al., 1987; Turner, 1987; Turner \& Oakes, 1989).

The results regarding central tendency judgments are also in line with research on accentuation. Within all the presented studies the likelihood for polarization of the stereotype (or accentuation of between-category differences) was greater than for attenuation. Among the research on the accentuation effect the work by Krueger and colleagues shares most similarity with the present line of research because it was conducted in the context of category learning and change (e.g., Krueger et al., 1989; Krueger \& Rothbart, 1990; for a review see Krueger, 1992). The present research, however, adds evidence that for category accentuation to occur one single deviant exemplar is sufficient. This demonstration sheds some light on the underlying processes as discussed in chapter 10.2. Furthermore, it also extends the scope by employing existing social stereotypes.

The lack of empirical support for contrast effects in the present studies, is in line with the fact that contrast effects have been demonstrated less often in previous research and therefore it was argued that assimilation seems to be the default process (Mussweiler, 2003). Presumably, the manipulation of one incongruent exemplar was too weak to trigger contrast effects and just attenuated the initial focus on similarities. The previously mentioned suggestion for further research (including a measurement of focus) could also help clarifying this question.

In sum, this dissertation provides evidence supporting the accentuation theory (Tajfel \& Wilkes, 1963) and the meta-contrast-ratio put forth by the self-categorization theory (Turner \& Oakes, 1989). It points to the context sensitivity of social stereotypes and thereby adds up to a long list of criticism on pure-prototype-based models (see Hamilton \& Sherman, 1994; Medin, 1989; Roth \& Shoben, 1983; Smith, 1990). As mentioned in the introduction, most researchers within social psychology recently allude to a hybrid model of category representation, which assumes that stereotype representation contains a mix of both, exemplars along with abstract knowledge (Judd \& Park, 1988; Park \& Hastie, 1987; Smith \& Medin, 1981; Smith \& Zárate, 1990). Which storage mode dominates was assumed to depend on diverse factors, such as familiarity with the category, or ingroup/outgroup status and valence of the stereotype (e.g., Sherman, 1996; Sherman, Klein, Lasky, \& Wyer, 1998). Furthermore, the kind of information people rely on when making a judgment (stored prototype vs. stored exem- 
plars) might also depend on the kind of judgment they have to make. When asked to judge whether a category possesses a certain trait or not, people might rely more on the prototype and might not retrieve single exemplars from memory. When asked to precisely judge to what degree members of a category possess that trait, they might additionally rely on stored exemplars because people are probably less familiar with making that kind of judgment. Perhaps, both, prototype and exemplars, are involved in the kind of judgment participants of the present studies had to give. Maybe people use the prototype for an initial, qualitative comparison between an exemplar and a category in order to decide whether to include or exclude the exemplar from the representation. Subsequently, they might retrieve exemplars from memory in order to come up with a precise judgment. Dependent on the outcome of the initial comparison, the retrieval might be guided by the salient exemplar or not. Obviously, the present data does not allow testing this idea, but it would fit the processes described in chapter 10.2.

\subsection{Limitations and Suggestions for Future Research}

One of the main limitations of the present studies is the fact that central mechanisms assumed to underlie the demonstrated effects need further investigation (for detailed discussion see chapter 10.2). A related shortcoming is the fact that the only study, in which pre- and post manipulation values could be matched, is Study 4. Study 4, however, is exceptional due to the fact that it employed an artificial stereotype about a group which was believed to be rather average on the central dimension. Unfortunately, certain questions could only be tested by employing a pre-post design and matching cases. For example, in order to test a mediational hypothesis, an assimilation index is needed. Another interesting question, which might be investigated in further studies is the impact of the original stereotype strength. Firstly, because this could be one way to study the impact of extremity of deviance, and secondly, to clarify the potential role of motivation in the process of stereotype polarization.

Although different paradigms have been used within the present set of studies, they still share some similarities which narrow generalizability. First, all studies dealt with the stereotype of different professions, or majors. This class of stereotypes has been chosen because it was expected that the present sample (students) should be familiar with these stereotypes. Furthermore, social desirability concerns were expected to be not as strong concerning these stereotypes because students were assumed to be kind of used to employ and, most importantly, to express them. Yet, the present research ques- 
tion developed upon a different, and perhaps socially more relevant categorization, namely according to cultural background (Muslims vs. Westerners). It can not be concluded with certainty that the same processes demonstrated in the present studies, are involved in stereotype maintenance and change related to different classes of stereotypes, which might differ in their relevance, function, or strength. At least, the present research suggests that the processes are independent of valence and content of the stereotype.

One reason for studying the effects with relatively less threatening stereotypes is the fact that the studies necessarily involve two sources of reactance. First, participants feel reluctant to express their stereotypes, and second, they react reluctant towards obvious sources of manipulation of their beliefs. By employing those stereotypes whose expression is less threatening, the first source of reactance was aimed to be decreased. In order to decrease the second source of reactance, complicated cover-stories and procedures (e.g., Study 7) were developed. Future research, might additionally, decrease awareness of the manipulation process by using more unobtrusive measures of stereotypes. For example, one way to unobtrusively measure the certainty or strength of the stereotype might be to measure its application. Study 7, for example, could have included in further rounds a re-concourse with another business student. By changing the game into a trust game one could assess whether the experience with a supercongruent business student affects trust in other business students. This way of measurement might be interesting to combine with the manipulation of incongruent information as well. Although, the incongruent information did not change the prototype in the present studies, it might change the certainty with which the stereotype is applied due to the increase in perceived variability. An additional way of studying the underlying processes in a way that might provoke less reactance, was suggested in the discussion of Part II and builds upon the finding that exemplar judgments are affected by the same kind of processes as stereotype judgments (see Study 5, but also Schwarz \& Bless, 1992). The reason why supercongruent exemplars are more likely to lead to an assimilation of the stereotype than incongruent exemplars, could possibly be studied as well by relying on exemplar judgments as an outcome variable.

\subsection{Conclusions}

"The first reference to "stereotype", in its modern, English use was in 1850, in the noun, meaning 'image perpetuated without change'" (Online Etymology Diction- 
ary). The present research, however, suggests that stereotypes do change. Stereotypes seem to be rigid in the sense that it might be hard to change their content; however, gradual change in the extremity or strength of the stereotype, seems to be more likely to occur. Present research suggests that stereotypes are especially likely to get polarized. The apparent asymmetry in processing of deviating information is probably one reason for the continuity of social stereotypes. One single supercongruent exemplar is likely to be taken as a good example for the category and to lead to a polarization of the stereotype. One single incongruent exemplar, on the contrary, is perceived as less typical and hardly influences the overall-stereotype. Given that social stereotypes have been shown to be a breeding ground for discriminative behaviour, the present results are rather worrying since they imply that stereotypes are very likely to get more and more extreme. As long as the media tends to focus on issues of crime and violence, press guidelines, mentioned in the introduction, are right by pointing to the jeopardy of stirring up prejudice when mentioning "religious, ethnic or other minority membership." To much pessimism seems, however, also inappropriate because the present studies also provided evidence that this process of stereotype polarization does not seem to be inevitable. Furthermore, not all stereotypes are negative in valence. Harvard professors, for example, might appreciate this tendency towards polarization whensoever one of Harvard's faculty members gets awarded with the Noble prize. 


\section{REFERENCES}

Abelson, R. P. (1983). Whatever became of consistency theory? Personality and Social Psychology Bulletin, 9(1), 37-64.

Abrams, D., Hogg, M. A., \& Marques, J. M. (2005). The social psychology of inclusion and exclusion. New York, NY, US: Psychology Press.

Abrams, D., Marques, J. M., Bown, N., \& Henson, M. (2000). Pro-norm and anti-norm deviance within and between groups. Journal of Personality and Social Psychology, 78, 906-912.

Allport, G. W. (1954). The nature of prejudice. Oxford, England: Addison-Wesley.

Ashmore, R. D., \& Del Boca, F. K. (1981). Conceptual approaches to stereotypes and stereotyping. In D. L. Hamilton (Ed.), Cognitive processes in stereotyping and intergroup behaviour (pp. 1-35). Hillsdale, NJ: Erlbaum.

Baron, R. M., \& Kenny, D. A. (1986). The moderator-mediator variable distinction in social psychological research: Conceptual, strategic, and statistical considerations. Journal of Personality and Social Psychology, 51, 1173-1182.

Bedeian, A. G., Mossholder, K. W., Touliatos, J., \& Barkman, A. I. (1986). The accountant's stereotype: An update for vocational counselors. The Career Development Quarterly, 35, 113-122.

Bless, H., Igou, E. R., Schwarz, N., \& Wänke, M. (2000). Reducing context effects by adding context information: The direction and size of context effects in political judgment. Personality and Social Psychology Bulletin, 26, 1036-1045.

Bless, H., \& Schwarz, N. (1998).Context effects in political judgment: assimilation and contrast as a function of categorization process. European Journal of Social Psychology, 28, 159-172. 
Bless, H., Schwarz, N., Bodenhausen, G. V., \& Thiel, L. (2001). Personalized versus generalized benefits of stereotype disconfirmation: Trade-offs in the evaluation of atypical exemplars and their social groups. Journal of Experimental Social Psychology, 37, 386-397.

Bless, H., \& Wänke, M. (2000). Can the same information be typical and atypical? How perceived typicality moderates assimilation and contrast in evaluative judgments. Personality and Social Psychology Bulletin, 26, 306-315.

Bodenhausen, G. V., Schwarz, N., Bless, H., \& Wänke, M. (1995). Effects of atypical exemplars on racial beliefs: Enlightened racism or generalized appraisals? Journal of Experimental Social Psychology, 31, 48-63.

Bodenhausen, G. V., \& Wyer, R. S. (1985). Effects of Stereotypes on Decision-Making and Information-Processing Strategies. Journal of Personality and Social Psychology, 48, 267-282.

Brewer, M. B., Dull, V., \& Lui, L. (1981). Perceptions of the elderly: Stereotypes as prototypes. Journal of Personality and Social Psychology, 41, 656-670.

Campbell, D. T. (1967). Stereotypes and the perception of group differences. American Psychologist, 22, 817-829.

Corneille, O., \& Judd, C. M. (1999). Accentuation and sensitization effects in the categorization of multifaceted stimuli. Journal of Personality and Social Psychology, 77, 927-941.

Cronbach, L. J. (1950). Further evidence on response sets and test design. Educational and Psychological Measurement, 10, 3-31.

Eiser, J. R. (1971). Enhancement of Contrast in Absolute Judgment of Attitude Statements. Journal of Personality and Social Psychology, 17, 1-10.

Eiser, J. R. (1990). Social Judgment. Pacific Grove, CA: Brooks/Cole. 
Eiser, J. R., \& Stroebe, W. (1972). Categorization and social judgment. Oxford, England: Academic Press.

Eiser, J. R., \& Van der Pligt, J. (1982). Accentuation and perspective in attitudinal judgment. Journal of Personality and Social Psychology, 42(2), 224-238.

Eysenck, M. W., \& Keane, M. T. (2000). Cognitive psychology: A student's handbook (4th ed.). New York, NY, US: Psychology Press.

Festinger, L. (1957). A theory of cognitive dissonance. Stanford, CA: Stanford University Press.

Fishbein, M., \& Lange, R. (1990). The effects of crossing the midpoint on belief change: A replication and extension. Personality and Social Psychology Bulletin, 16, 189-199.

Fiske, S. T., Cuddy, A. J. C., Glick, P., \& Xu, J. (2002). A model of (often mixed) stereotype content: Competence and warmth respectively follow from perceived status and competition. Journal of Personality and Social Psychology, 82, 878902.

Fiske, S. T., Neuberg, S. L., Beattie, A. E., \& Milberg, S. J. (1987). Category-Based and Attribute-Based Reactions to Others - Some Informational Conditions of Stereotyping and Individuating Processes. Journal of Experimental Social Psychology, $23,399-427$.

Fiske, S. T., \& Taylor, S. E. (1991). Social cognition (2nd ed.). New York, NY, England: Mcgraw-Hill Book Company.

Fiske, S. T., Xu, J., Cuddy, A. C., \& Glick, P. (1999). (Dis)respecting versus (dis)liking: Status and interdependence predict ambivalent stereotypes of competence and warmth. Journal of Social Issues, 55, 473-489. 
Ford, T. E., \& Stangor, C. (1992). The role of diagnosticity in stereotype formation: Perceiving group means and variances. Journal of Personality and Social Psychology, 63, 356-367.

Garcia-Marques, L., \& Mackie, D. M. (1999). The impact of stereotype-incongruent information on perceived group variability and stereotype change. Journal of Personality and Social Psychology, 77, 979-990.

Goldstone, R. L. (1994). Influences of Categorization on Perceptual Discrimination. Journal of Experimental Psychology: General, 123(2), 178-200.

Goldstone, R. L. (1995). Effects of categorization on color perception. Psychological Science, 6(5), 298-304.

Goldstone, R. L. (1996). Isolated and interrelated concepts. Memory \& Cognition, 24(5), 608-628.

Goldstone, R. L., Steyvers, M., \& Rogosky, B. J. (2003). Conceptual interrelatedness and caricatures. Memory \& Cognition, 31, 169-180.

Hamburger, Y. (1994). The contact hypothesis reconsidered: Effects of the atypical outgroup member on the outgroup stereotype. Basic and Applied Social Psychology, 15, 339-358.

Hamilton, D. L., \& Sherman, J. W. (1994). Stereotypes. In R. S. Wyer, Jr., \& T. K. Srull (Eds.), Handbook of Social Cognition (2 ${ }^{\text {nd }}$ ed., pp. 59-82). Hillsdale, NJ: Erlbaum.

Haslam, S. A., Oakes, P. J., McGarty, C., Turner, J. C., \& Onorato, R. S. (1995). Contextual Changes in the Prototypicality of Extreme and Moderate Outgroup Members. European Journal of Social Psychology, 25, 509-530.

Heider, F. (1946). Attitudes and cognitive organization. Journal of Psychology: Interdisciplinary and Applied, 21, 107-112. 
Hewstone, M. (1990). The 'ultimate attribution error'? A review of the literature on intergroup causal attribution. European Journal of Social Psychology, 20(4), 311335 .

Hewstone, M. (1994). Revision and change of stereotypic beliefs: In search for the elusive subtyping model. In W. Stroebe \& M. Hewstone (Eds.), European review of social psychology (Vol. 5, pp. 69-109). Chichester, UK: Wiley.

Hewstone, M., \& Brown, R. (1986). Contact is not enough: An intergroup perspective on the 'contact hypothesis.' In M. Hewstone, \& R. Brown (Ed), Contact and conflict in intergroup encounters (pp. 1-44). Cambridge, MA, US: Basil Blackwell.

Hewstone, M., \& Hamberger, J. (2000). Perceived variability and stereotype change. Journal of Experimental Social Psychology, 36, 103-124.

Hewstone, M., Hassebrauck, M., Wirth, A., \& Waenke, M. (2000). Pattern of disconfirming information and processing instructions as determinants of stereotype change. British Journal of Social Psychology, 39, 399-411.

Higgins, E. T. (1996). Knowledge activation: Accessibility, applicability, and salience. New York, NY, US: Guilford Press.

Hilton, J. L., \& von Hippel, W. (1996). Stereotypes. Annual Review of Psychology, 47, 237-271.

Jäger, M., Cleve, G., Ruth, I., \& Jäger, S. (1998). Von deutschen Einzeltätern und ausländischen Banden. Medien und Straftaten. Mit Vorschlägen zur Vermeidung diskriminierender Berichterstattung, Duisburg: Unrast Verlag.

Johnston, L., \& Hewstone, M. (1992). Cognitive models of stereotype change: III. Subtyping and the perceived typicality of disconfirming group members. Journal of Experimental Social Psychology, 28, 360-386. 
Johnston, L., Hewstone, M., Pendry, L., \& Frankish, C. (1994). Cognitive models of stereotype change: IV. Motivational and cognitive influences. European Journal of Social Psychology, 24, 237-265.

Jost, J. T., \& Banaji, M. R. (1994). The Role of Stereotyping in System-Justification and the Production of False Consciousness. British Journal of Social Psychology, 33, 1-27.

Judd, C. M., \& Kenny, D. A. (1981). Process analysis: Estimating mediation in treatment evaluations. Evaluation Review, 5, 602-619.

Judd, C. M., \& Park, B. (1988). Out-group homogeneity: Judgments of variability at the individual and group levels. Journal of Personality and Social Psychology, 54, 778-788.

Judd, C. M., Ryan, C. S., \& Park, B. (1991). Accuracy in the judgment of in-group and out-group variability. Journal of Personality and Social Psychology, 61, 366379.

Kenny, D. A., Kashy, D. A., \& Bolger, N. (1998). Data analysis in social psychology. New York, NY, US: McGraw-Hill.

Krueger, J. (1991). Accentuation effects and illusory change in exemplar-based category learning. European Journal of Social Psychology, 21(1), 37-48.

Krueger, J. (1992). On the overestimation of between-group differences. In W.Stroebe \& M. Hewstone (Eds.), European review of social psychology, Vol. 3 (pp. 3156). John Wiley \& Sons.

Krueger, J., \& Clement, R. W. (1994). Memory-based judgments about multiple categories: A revision and extension of Tajfel's accentuation theory. Journal of Personality and Social Psychology, 67, 35-47. 
Krueger, J., \& Rothbart, M. (1990). Contrast and accentuation effects in category learning. Journal of Personality and Social Psychology, 59, 651-663.

Krueger, J., Rothbart, M., \& Sriram, N. (1989). Category learning and change: Differences in sensitivity to information that enhances or reduces intercategory distinctions. Journal of Personality and Social Psychology, 56, 866-875.

Kunda, Z., \& Oleson, K. C. (1995). Maintaining stereotypes in the face of disconfirmation: Constructing grounds for subtyping deviants. Journal of Personality and Social Psychology, 68, 565-579.

Kunda, Z., \& Oleson, K. C. (1997). When exceptions prove the rule: How extremity of deviance determines the impact of deviant examples on stereotypes. Journal of Personality and Social Psychology, 72, 965-979.

Kunda, Z., \& Sherman-Williams, B. (1993). Stereotypes and the construal of individuating information. Personality and Social Psychology Bulletin, 19, 90-99.

Lange, R., \& Fishbein, M. (1983). Effects of category differences on belief change and agreement with the source of a persuasive communication. Journal of Personality and Social Psychology, 44, 933-941.

Leach, C. W. (2006). The meaning of prejudice. Unpublished manuscript, University of Sussex, Brighton, England.

Linville, P. W., \& Fischer, G. W. (1993). Exemplar and abstraction models of perceived group variability and stereotypicality. Social Cognition, 11, 92-125.

Linville, P. W., Fischer, G. W., \& Salovey, P. (1989). Perceived distributions of the characteristics of in-group and out-group members: Empirical evidence and a computer simulation. Journal of Personality and Social Psychology, 57, 165188.

Lippmann, W. (1922). Public Opinion. New York: Hartcourt, Brace. 
Macrae, C. N., Milne, A. B., \& Bodenhausen, G. V. (1994). Stereotypes As EnergySaving Devices - A Peek Inside the Cognitive Toolbox. Journal of Personality and Social Psychology, 66, 37-47.

Maurer, K. L., Park, B., \& Rothbart, M. (1995). Subtyping versus subgrouping processes in stereotype representation. Journal of Personality and Social Psychology, $69,812-824$.

McCauley, C., \& Stitt, C. L. (1978). Individual and Quantitative Measure of Stereotypes. Journal of Personality and Social Psychology, 36, 929-940.

McCauley, C., Stitt, C. L., \& Segal, M. (1980). Stereotyping: From prejudice to prediction. Psychological Bulletin, 87, 195-208.

McCloskey, M. E., \& Glucksberg, S. (1978). Natural Categories - Well Defined Or Fuzzy Sets. Memory \& Cognition, 6, 462-472.

McGarty, C. (1999). Categorization in social psychology. London. Sage.

McGarty, C. \& Penny, R. E. (1988). Categorization, accentuation and social judgement. British Journal of Social Psychology, 27, 147-157.

Medin, D. L. (1989). Concepts and conceptual structure. American Psychologist, 44, 1469- 1481.

Medin, D. L., Goldstone, R. L., \& Gentner, D. (1993). Respects for Similarity. Psychological Review, 100, 254-278.

Medin, D. L., Lynch, E. B., \& Solomon, K. O. (2000). Are there kinds of concepts? Annual Review of Psychology, 51, 121-147.

Medin, D. L., \& Schaffer, M. M. (1978). Context Theory of Classification Learning. Psychological Review, 85, 207-238. 
Mullen, B., Brown, R., \& Smith, C. (1992). Ingroup bias as a function of salience, relevance, and status: An integration. European Journal of Social Psychology, 22, $103-122$.

Murphy, G. L., \& Medin, D. L. (1985). The role of theories in conceptual coherence. Psychological Review, 92, 289-316.

Mussweiler, T. (2001). 'Seek and ye shall find': Antecedents of assimilation and contrast in social comparison. European Journal of Social Psychology, 31, 499-509.

Mussweiler, T. (2003). Comparison processes in social judgment: Mechanisms and consequences. Psychological Review, 110, 472-489.

Mussweiler, T., Rüter, K., \& Epstude, K. (2004). The Ups and Downs of Social Comparison: Mechanisms of Assimilation and Contrast. Journal of Personality and Social Psychology, 87, 832-844.

Mussweiler, T., \& Strack, F. (1999). Hypothesis-consistent testing and semantic priming in the anchoring paradigm: A selective accessibility model. Journal of Experimental Social Psychology, 35, 136-164.

Mussweiler, T., \& Strack, F. (2000). Consequences of social comparison. Selective accessibility, assimilation, and contrast. In J. Suls, \& S. C. Wheeler (Eds.), Handbook of social comparison: Theory and research (pp. 253-270). New York: Kluwer Academic/Plenum Publishers.

Nemeth, C., \& Endicott, J. (1976). Midpoint As An Anchor - Another Look at Discrepancy of Position and Attitude-Change. Sociometry, 39, 11-18.

Newcomb, T. M. (1947). Autistic hostility and social reality. Human Relations, 1, 6986. 
Nisbett, R. E., Krantz, D. H., Jepson, C., \& Kunda, Z. (1983). The use of statistical heuristics in everyday inductive reasoning. Psychological Review, 90, 339-363.

Nisbett, R. E., Zukier, H., \& Lemley, R. E. (1981). The dilution effect: Nondiagnostic information weakens the implications of diagnostic information. Cognitive Psychology, 13, 248-277.

Nosofsky, R. M. (1986). Attention, similarity, and the identification-categorization relationship. Journal of Experimental Psychology: General, 115, 39-57.

Nosofsky, R. M. (1991). Typicality in logically defined categories: Exemplar-similarity versus rule instantiation. Memory \& Cognition, 19(2), 131-150.

Oakes, P. J., Haslam, S. A., \& Reynolds, K. J. (1999). Social categorization and social context: Is stereotype change a matter of information or of meaning? In D.Abrams \& M. A. Hogg (Eds.), Social identity and social cognition (pp. 5579). Blackwell Publishing.

Oakes, P., Haslam, S. A., \& Turner, J. C. (1998). The role of prototypicality in group influence and cohesion: Contextual variation in the graded structure of social categories. Thousand Oaks, CA, US: Sage Publications, Inc.

Online Etymology Dictionary (2007). Retrieved September 20, 2007, from http://www.etymonline.com/index.php?term=stereotype

O'Sullivan, C. S., \& Durso, F. T. (1984). Effect of Schema-Incongruent Information on Memory for Stereotypical Attributes. Journal of Personality and Social Psychology, 47, 55-70.

Palmeri, T. J., \& Nosofsky, R. M. (2001). Central tendencies, extreme points, and prototype enhancement effects in ill-defined perceptual categorization. The Quarterly Journal of Experimental Psychology A: Human Experimental Psychology, 54, 197-235. 
Paolini, S., Hewstone, M., Rubin, M., \& Pay, H. (2004). Increased group dispersion after exposure to one deviant group member: Testing Hamburger's model of member-to-group generalization. Journal of Experimental Social Psychology, 40, 569-585.

Park, B., \& Hastie, R. (1987). Perception of variability in category development: Instance- versus abstraction-based stereotypes. Journal of Personality and Social Psychology, 53, 621-635.

Park, B., Ryan, C. S., \& Judd, C. M. (1992). Role of meaningful subgroups in explaining differences in perceived variability for in-groups and out-groups. Journal of Personality and Social Psychology, 63, 553-567.

Park, B., Wolsko, C., \& Judd, C. M. (2001). Measurement of subtyping in stereotype change. Journal of Experimental Social Psychology, 37, 325-332.

Peters, E., \& Rothbart, M. (2000). Typicality can create, eliminate, and reverse the dilution effect. Personality and Social Psychology Bulletin, 26, 177-187.

Posner, M. I., \& Keele, S. W. (1970). Retention of abstract ideas. Journal of Experimental Psychology, 83, 304-308.

Quattrone, G. A., \& Jones, E. E. (1980). The perception of variability within in-groups and out-groups: Implications for the law of small numbers. Journal of Personality and Social Psychology, 38, 141-152.

Queller, S., \& Smith, E. R. (2002). Subtyping versus bookkeeping in stereotype learning and change: Connectionist simulations and empirical findings. Journal of Personality and Social Psychology, 82, 300-313.

Quinn, K. A., \& Macrae, C. N. (2005). Categorizing Others: The Dynamics of Person Construal. Journal of Personality and Social Psychology, 88, 467-479. 
Reed, S. K. (1972). Pattern recognition and categorization. Cognitive Psychology, 3, $382-387$

Richards, Z., \& Hewstone, M. (2001). Subtyping and subgrouping: Processes for the prevention and promotion of stereotype change. Personality and Social Psychology Review, 5, 52-73.

Rosch, E. (1975).Cognitive representations of semantic categories. Journal of Experimental Psychology, 104, 192-233.

Rosch, E. (1978). Principles of categorization. In E. Rosch \& B. B. Lloyd (Eds.). Cognition and categorization (pp. 27-48). Hillsdale, NJ: Erlbaum.

Rosch, E., \& Mervis, C. B. (1975). Family Resemblances - Studies in Internal Structure of Categories. Cognitive Psychology, 7, 573-605.

Roth, E. M., \& Shoben, E. J. (1983). The Effect of Context on the Structure of Categories. Cognitive Psychology, 15, 346-378.

Rothbart, M. K. (1981). Measurement of Temperament in Infancy. Child Development, $52,569-578$.

Rothbart, M. (1996). Category-exemplar dynamics and stereotype change. International Journal of Intercultural Relations, 20, 305-321.

Rothbart, M., Evans, M., \& Fulero, S. (1979). Recall for confirming events: Memory processes and the maintenance of social stereotypes. Journal of Experimental Social Psychology, 15, 343-355.

Rothbart, M. \& John, O. P. (1985). Social categorization and behavioral episodes: A cognitive analysis of the effects of intergroup contact. In C. Stangor (Ed.), Stereotypes and prejudice: Essential readings (pp. 419-434). Psychology Press. 
Rothbart, M., \& Lewis, S. (1988). Inferring category attributes from exemplar attributes: Geometric shapes and social categories. Journal of Personality and Social Psychology, 55, 861-872.

Rothbart, M., \& Park, B. (1986). On the confirmability and disconfirmability of trait concepts. Journal of Personality and Social Psychology, 50, 131-142.

Rothbart, M., Sriram, N., \& Davis-Stitt, C. (1996). The retrieval of typical and atypical category members. Journal of Experimental Social Psychology, 32, 309-336.

Schneider, D. (2004). Stereotypes. New York: The Guilford Press.

Schwarz, N., \& Bless, H. (1992). Scandals and the public's trust in politicians: Assimilation and contrast effects. Personality and Social Psychology Bulletin, 18, $574-$ 579 .

Schwarz, N., \& Bless, H. (2007). Mental Construal Processes: The Inclusion/Exclusion Model. New York, NY, US: Psychology Press.

Seta, J. J., Seta, C. E., \& McElroy, T. (2003). Attributional biases in the service of stereotype maintenance: A schema-maintenance through compensation analysis. Personality and Social Psychology Bulletin, 29, 151-163.

Sherman, J. W. (1996). Development and Mental Representation of Stereotypes. Journal of Personality and Social Psychology, 70(6), 1126-1141.

Sherman, J. W., Klein S. B., Laskey, A., \& Wyer, N. A. (1998). Intergroup bias in group judgment processes: The role of behavioural memories. Journal of Experimental Social Psychology, 34, 51-65.

Shortnews (2007). 14.01.07. Retrieved September 20, 2007, from http://www.shortnews.de/start.cfm?id=651645 
Skowronski, J. J., \& Carlston, D. E. (1989). Negativity and extremity biases in impression formation: A review of explanations. Psychological Bulletin, 105, 131-142.

Smith, E. (1990).Concept and process specificity in the effects of prior experiences. Target Article in T. K. Srull \& R. S. Wyer (Eds.), Advances in social cognition (Vol. 3, pp. 1-60). Hillsdale, NJ: Lawrence Erlbaum Association.

Smith, E., \& Medin, D. L. (1981). Categories and Concepts. Cambridge, MA: Harvard University Press.

Smith, E. R., \& Zárate, M. A. (1990). Exemplar and prototype use in social categorization. Social Cognition, 8, 243-262.

Smith, E. R., \& Zárate, M. A. (1992). Exemplar-Based Model of Social Judgment. Psychological Review, 99, 3-21.

Smith, E. R., \& Queller, S. (2004). Mental Representations. Malden, MA, US: Blackwell Publishing.

Smith, L. (1998). On the development of mental representation. Developmental Review, $18,202-227$.

Snyder, M., \& Miene, P. K. (1994). Stereotyping of the Elderly - A FunctionalApproach. British Journal of Social Psychology, 33, 63-82.

Spencer, S. J., Zanna, M. P., \& Fong, G. T. (2005). Establishing a causal chain: Why experiments are often more effective than mediational analyses in examining psychological processes. Journal of Personality and Social Psychology, 89(6), 845-851.

Tajfel, H. (1969). Cognitive aspects of prejudice. Journal of Social Issues, 25(4), 79-97.

Tajfel, H., \& Wilkes, A. L. (1963). Classification and quantitative judgement. British Journal of Psychology, 54, 101-114. 
The German Press Council (2006). German Press Code. Retrieved September 20, 2007, from http://www.presserat.de/uploads/media/Press Code.pdf

Tropp, L. R., \& Pettigrew, T. F. (2005). Relationships between intergroup contact and prejudice among minority and majority status groups. Psychological Science, 16, 951-957.

Turner, J.C. (1987). A self-categorization theory. In J. C. Turner, M. A. Hogg, P. J. Oakes, S. D. Reicher, \& M. S. Wetherell (Eds.), Rediscovering the social group: A self-categorization theory (pp. 68-88). Oxford, England: Basil Blackwell.

Turner, J. C., Hogg, M. A., Oakes, P. J., Reicher, S. D., \& Wetherell, M. S. (1987). Rediscovering the social group: A self-categorization theory. Cambridge, MA, US: Basil Blackwell.

Turner, J. C. \& Oakes, P. J. (1989). Self-categorization theory and social influence. Hillsdale, NJ, England: Lawrence Erlbaum Associates, Inc.

Tversky, A. (1977). Features of similarity. Psychological Review, 84, 327-352.

Tversky, A. \& Kahneman, D. (1973). Availability - Heuristic for Judging Frequency and Probability. Cognitive Psychology, 5, 207-232.

Weber, R., \& Crocker, J. (1983). Cognitive processes in the revision of stereotypic beliefs. Journal of Personality and Social Psychology, 45, 961-977.

Wilder, D. A. (1984). Intergroup contact: The typical member and the exception to the rule. Journal of Experimental Social Psychology, 20, 177-194.

Wolsko, C., Park, B., Judd, C. M., \& Bachelor, J. (2003). Intergroup contact: Effects on group evaluations and perceived variability. Group Processes \& Intergroup Relations, 6, 93-110. 
Wyer, N. A., Sadler, M. S., \& Judd, C. M. (2002). Contrast effects in stereotype formation and change: the role of comparative context. Journal of Experimental Social Psychology, 38, 443-458.

Yzerbyt, V. Y., Coull, A., \& Rocher, S. J. (1999). Fencing off the deviant: The role of cognitive resources in the maintenance of stereotypes. Journal of Personality and Social Psychology, 77, 449-462. 


\section{APPENDIX}

Instance of manipulation: Study 5 incongruent (similar manipulation used in

Study $3,4,5$, and 6 )

\section{Individuelles Testergebnis}

Teilnehmer-Code: KH476DF

Studiengang: Studium der Wirtschaftswissenschaften

(Abschlussnote: gut)

\section{Problemlösefähigkeit}

Befähigung zu abstraktem Denken; logisches Schlussfolgern

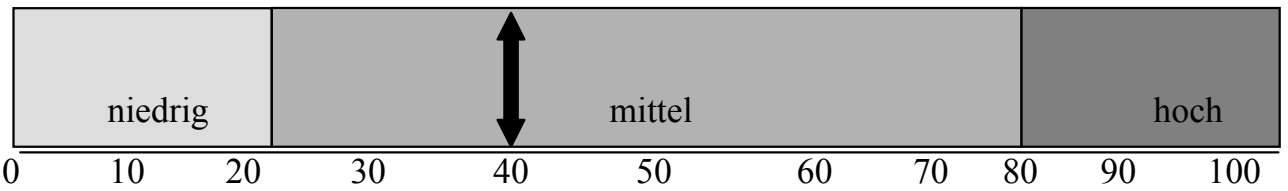

\section{Streben nach Erfolg}

Ehrgeiz; Bereitschaft für Erfolg extrem viel zu leisten.

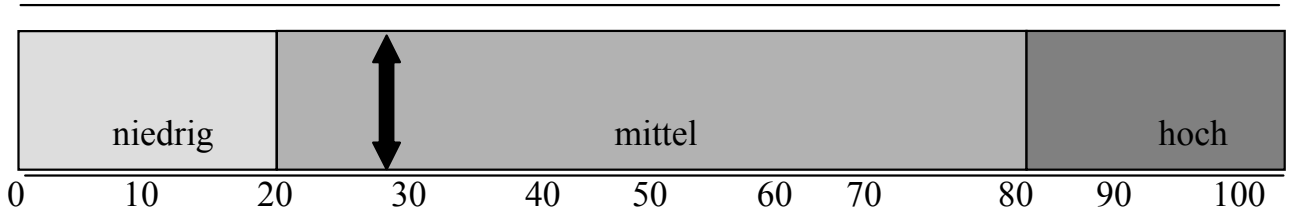

\section{Verständnis}

Fähigkeit komplexe Sachverhalte möglichst schnell zu erfassen

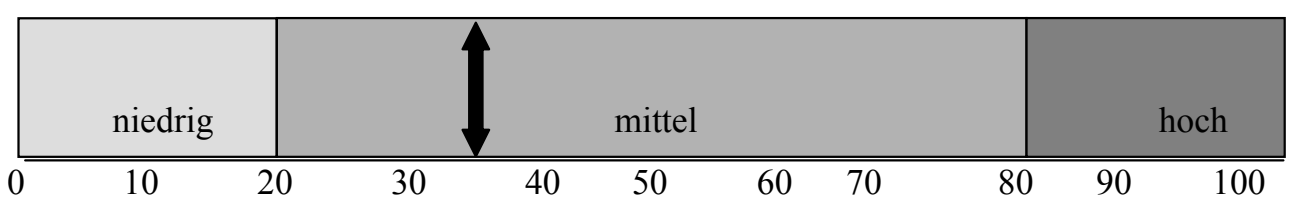




\section{SUMMARY}

Given the immense amount of research on stereotypes, one might expect that the nature of stereotypes and the processes involved in stereotype change are fairly well understood. Yet, some questions remain unsolved. For example, research on stereotype change has so far mainly focused on stereotype attenuation and accordingly studied the impact of stereotype-incongruent exemplars, that is exemplars that are contradicting the stereotype of their category. Stereotype-inconsistent exemplars, however, are just one side of the coin. The present dissertation investigated whether information that deviates in one or the other direction from the stereotype is processed in the same way.

Research so far has demonstrated that stereotype-incongruent exemplars of a category typically do not change a stereotype (,,subtyping“; e.g., Johnston \& Hewstone, 1992; Johnston, Hewstone, Pendry, \& Frankish, 1994; Kunda \& Oleson, 1995; Weber \& Crocker, 1983; for an overview see Hewstone, 1994; Hilton \& von Hippel, 1996). Yet, we do not know whether this is also the case with exemplars deviating in direction of the stereotype, which means the exemplar exceeds the stereotypical expectation (supercongruent). The present research investigated whether supercongruent and incongruent information trigger the same kind of processes or not, and thereby aimed at broadening the knowledge about stereotype maintenance and change.

To date, research has demonstrated that one of the main determinants whether an exemplar will exert influence on the over-all stereotype or not, is the perceived typicality of the exemplar (e.g., Johnston \& Hewstone, 1992; Kunda \& Oleson, 1997; Rothbart \& John, 1985; Rothbart \& Lewis, 1988; Wilder, 1984). Whether supercongruent and incongruent exemplars differ in perceived typicality was examined within the present dissertation. Self-categorization-theory has postulated that perceived typicality of an exemplar depends upon an intra- and intergroup comparison (meta-contrast-principle; Turner \& Oakes, 1989). Accordingly, the similarity of an exemplar to other members of the same category as well as to members of contrasting categories should be involved in this judgement. Incongruent exemplars have been defined as deviating in the direction of comparison categories (Brewer, Lui, \& Dull, 1981). Supercongruent exemplars can be defined accordingly as those exemplars that deviate in the opposite direction, away from comparison categories. Supercongruent exemplars should therefore be perceived as more typical than incongruent exemplars because the latter one share more similarity 
with members of contrasting categories. A series of studies has been conducted to test this assumption.

All of the present studies were preceded by a pre-test which assessed the average expectancies concerning a certain category in relation to a certain comparison category. Participants of this pretest were asked to express their expectancies numerically by indicating expected IQ-values (Study 1,2), or test points in competence and social competence related tests (Studies 3, 4, 5, and 6), or expected points shared in a game by their co-player (Study 7). Based on these data, deviant exemplars were constructed which either matched the expectations for the comparison category (incongruent), or deviated with the same amount from the prototype but in the opposite direction (supercongruent). ${ }^{28}$

The first assumption concerned with differences in perceived typicality was tested and supported in Study 1, 3, 4, and 5. Supercongruent exemplars were perceived consistently as more typical than incongruent exemplars. Employing different kinds of stereotypes, with different valence and content dimensions increased the generalizability of the finding. Study 4 furthermore supported the meta-contrast-principle by demonstrating that even the same exemplar is perceived as typical to different degrees depending on the kind of comparison category. Still, people seem to be able to detect a deviance in a supercongruent direction as soon as their attention is drawn to it. Supercongruent exemplars were judged as less typical than congruent exemplars. This difference was especially pronounced when participants had to judge perceived deviance (Study 1) and when typicality was measured before the stereotype assessment, thus before they had the chance to assimilate their stereotype to the supercongruent exemplar (Study 5).

Beyond differences in perceived typicality, I was especially interested in the impact of these kinds of exemplars on the stereotype of their category. It has been hypothesized that supercongruent exemplars should lead to more assimilation of the stereotype compared with incongruent exemplars. This assumption is based on two lines of argumentation: 1) Differences in perceived typicality should lead to different amounts of generalization, and 2) the general tendency to accentuate differences between groups speaks to a stronger influence of supercongruent exemplars compared with incongruent exemplars (accentuation theory; for a review see Krueger, 1992).

Results of Studies 2, 3, 4, and 5 supported the assumption that stereotypes are more likely to change in direction of supercongruent exemplars than in direction of in-

\footnotetext{
${ }^{28}$ Slightly different procedures have been employed in Study 6 and 7 (e.g., no incongruent condition).
} 
congruent exemplars, and that supercongruent information changes perceived differences between group whereas incongruent information does not (Study 2). Furthermore, Study 4 provided evidence that this asymmetry is not just due to the greater extremity of supercongruent information compared with incongruent information. However, results of the same study did not support the idea that differences in perceived typicality are responsible for the asymmetric impact the exemplars have. Hence, the question remained, why supercongruent exemplars exert a bigger influence than incongruent exemplars. Results of Study 5 and 6 contain first hints concerning this issue.

In order to shed some further light on the underlying processes, Studies 5 and 6 investigated the circumstances in which no assimilation to a supercongruent exemplar occurs. It was assumed that a supercongruent exemplar posits a quantitative deviance. Both exemplar and prototype share the same attributes, their difference is just a matter of degree. Incongruent exemplars, though, oftentimes do not possess the stereotypic attribute at all. Their deviance therefore can be understood as a qualitative deviance. Presumably, a qualitative deviance is easier recognized than a quantitative deviance and therefore should lead to a perception of less psychological closeness between both stimuli (exemplar and category). According to the selective accessibility model (Mussweiler \& Strack, 2000) a comparison that focuses on dissimilarities should follow, and lead to a contrast effect, whereas a comparison that focuses on similarities should result from a quantitative (supercongruent) deviance, which in turn should lead to an assimilation of the stereotype. If this assumption holds, any manipulation which draws people attention towards the deviance of supercongruent exemplars, should impede generalization from the exemplar to the category. And indeed, no generalization occurred in Study 5 and 6, when participants had to judge the deviance of the exemplar from the category before the stereotype assessment took place.

As stereotypes often involve negative consequences, like discrimination, it is rather worrying to learn, that they easily get polarized. Study 7 was conducted to investigate a simple mechanism that could prevent generalization of supercongruent exemplars to the category. Previous research in the field of stereotype change has shown that additional, neutral information about an incongruent exemplar normally entails that the stereotype is not assimilated to the exemplar. Additional information is used in this context to justify subtyping. Study 7 demonstrated a similar result for supercongruent exemplars, although different mechanism might form the basis of this effect. 
In sum, the present studies contribute to the understanding of stereotypes. Previous research dealt with the impact of incongruent exemplars. The present dissertation broadens that knowledge by studying the impact of supercongruent exemplars and comparing it to that of incongruent ones. Replicating previous research, the impact of incongruent exemplars was rather small within the present set of studies. Supercongruent exemplars, by contrast, generally led to an assimilation and thereby polarization of the stereotype. This process, however, does not seem to be inevitable. The present studies additionally provided first hints how the polarization of a stereotype due to supercongruent information could be counteracted. 


\section{ZUSAMMENFASSUNG}

Angesicht einer Vielzahl an publizierten Studien zum Thema Stereotype, sollte man annehmen, dass das Wesen von Stereotypen und die Prozesse bei der Veränderung von Stereotypen relativ wohlverstanden sind. Einige Fragen sind jedoch noch immer ungeklärt. So wurde beispielsweise die Veränderung von Stereotypen bislang nur in eine Richtung untersucht: Wie sehr können Mitglieder einer Kategorie, die dem Stereotyp widersprechen (inkongruent), das Bild der Kategorie beeinflussen? Dabei haben sich Stereotype als relativ resistent gegenüber widersprechender Information erwiesen (siehe Hewstone, 1994; Hilton \& von Hippel, 1996). Ein Grund für diese Änderungsresistenz könnte eine Asymmetrie in der Verarbeitung von Information sein, die von der stereotypen Erwartung abweicht. Inkongruente Information wird häufig als Ausnahme behandelt und trägt nichts zu einer Veränderung bei (z.B. Johnston \& Hewstone, 1992; Johnston, Hewstone, Pendry \& Frankish, 1994; Kunda \& Oleson, 1995; Weber \& Crocker, 1983). Doch was passiert eigentlich wenn Exemplare, die stereotypen Erwartungen eindeutig übertreffen, also noch extremer sind als erwartet (superkongruent)? Wie sehr sind Menschen in der Lage, eine Abweichung in superkongruenter Richtung als solche zu erkennen? In der vorliegenden Dissertation wurde untersucht, ob superkongruente und inkongruente Information vergleichbare Prozesse auslöst, mit dem Ziel das Wissen über die Aufrechterhaltung und Veränderung von Stereotypen zu erweitern.

Die bisherige Forschung hat gezeigt, dass eine Generalisierung von einem Exemplar auf die jeweilige Kategorie vor allem dann stattfindet, wenn das Exemplar als typisch für die Kategorie wahrgenommen wird (z.B. Johnston \& Hewstone, 1992; Kunda \& Oleson, 1997; Rothbart \& John, 1985; Rothbart \& Lewis, 1988; Wilder, 1984). Entsprechend wurde innerhalb der vorliegenden Arbeit zunächst untersucht, ob sich inkongruente und superkongruente Exemplare in ihrer wahrgenommenen Typikalität unterscheiden. Innerhalb der Selbstkategorisierungstheorie wurde postuliert, dass die Typikalität eines Exemplars sowohl von einem Intragruppenvergleich als auch von einem Intergruppenvergleich abhängt (Meta-Kontrast-Prinzip; Turner \& Oakes, 1989). Demnach wird die Ähnlichkeit eines Exemplars zu anderen Mitgliedern derselben Kategorie, als auch zu den Mitgliedern anderer Kategorien beurteilt. Inkongruente Exemplare wurden bereits früher als diejenigen Exemplare definiert, die in Richtung relevanter Vergleichskategorien vom Prototyp abweichen (Brewer, Lui, \& Dull, 1981). Superkongruente Exemplare lassen sich entsprechend als solche Exemplare verstehen, die in die 
entgegen gesetzte Richtung, weg von Vergleichskategorien, abweichen. Demnach sollten superkongruente Exemplare als typischer wahrgenommen werden als inkongruente Exemplare, denn die Ähnlichkeit zu Nicht-Mitgliedern ist bei letzteren größer. Eine Reihe von Studien wurde durchgeführt, um diese Annahme zu testen.

Allen Studien der vorliegenden Arbeit ging jeweils ein Vortest voraus, der die stereotype Erwartung bezüglich einer Kategorie im Vergleich zu einer anderen Kategorie erfasste. Teilnehmer dieser Vortests sollten ihre Erwartungen numerisch ausdrücken, bezüglich IQ-Werte (Studie 1,2) oder Testwerte in Kompetenz oder Sozialkompetenztests (Studie 3, 4, 5, 6), oder erwartete Punkteaufteilung in einem Spiel (Studie 7). Anhand dieser Erwartungswerte wurden abweichende Exemplare konstruiert, die entweder dem Erwartungswert der Vergleichsgruppe entsprachen (inkongruent) oder in gleichem Ausmaß, aber in entgegen gesetzter Richtung vom Prototyp abwichen (superkongruent).

Diese Annahme wurden in den Studien 1, 3, 4 und 5 getestet und bestätigt. Superkongruente Exemplare wurden durchweg als typischer wahrgenommen als inkongruente Exemplare mit derselben objektiven Abweichung vom Prototyp. Dass diesen Studien ganz unterschiedliche Stereotype, sowohl mit positiver als auch mit negativer Valenz, zu Grunde lagen, erhöht die Generalisierbarkeit der vorliegenden Befunde. Studie 4 bekräftigt ebenfalls das Meta-Kontrast-Prinzip. Das gleiche Exemplar wurde in dieser Studie als unterschiedlich typisch bewertet je nachdem welche Vergleichskategorie salient war. Menschen scheinen jedoch in der Lage, auch eine Abweichung in Richtung des Stereotyps zu erkennen, wenn ihre Aufmerksamkeit darauf gelenkt wird. Superkongruente Exemplare wurden in Studie 1 und 5 als weniger typisch bewertet als kongruente Exemplare. Dieser Unterschied trat besonders dann auf, wenn nach der wahrgenommenen Abweichung gefragt wurde (Studie 1), oder die Typikalität vor der Stereotypbewertung abgefragt wurde, bevor die Wahrnehmenden also die Chance hatten, ihr Stereotyp an das superkongruente Exemplar anzupassen (Studie 5).

Über Unterschiede in der Typikalität hinaus interessierte mich jedoch vor allem der Einfluss der Exemplare auf das jeweilige Stereotyp. Es wurde vorhergesagt, dass superkongruente Exemplare viel eher zu einer Anpassung des Stereotypes an das Exemplar führen sollten als inkongruente Exemplare. Diese Annahme gründet auf verschiedenen Argumentationssträngen: 1) Unterschiede in der wahrgenommenen Typikalität sollten zu einem unterschiedlich starken Einfluss führen; und 2) das generelle Streben, Unterschiede zwischen sozialen Gruppen zu akzentuieren, sollte zu einem stärke- 
ren Einfluss der superkongruenten Exemplare führen (Akzentuierungstheorie, Krueger, 1992).

Ergebnisse der Studien 2, 3, 4 und 5 bestätigen die Annahme, dass Stereotype sich leichter in Richtung superkongruenter Exemplare verändern als in Richtung inkongruenter Exemplare, und dass wahrgenommene Unterschiede zwischen Gruppen sich durch superkongruente Exemplare vergrößern, während inkongruente Exemplare keinen Einfluss darauf ausüben. Ergebnisse der Studie 4 zeigen darüber hinaus, dass diese Asymmetrie nicht nur über die unterschiedliche Extremität der Exemplare zu erklären ist. Allerdings weisen die Ergebnisse derselben Studie auch daraufhin, dass die unterschiedliche Typikalität der beiden Exemplare nicht die Ursache für den unterschiedlichen Einfluss ist. Da auch die Akzentuierungstheorie keine eindeutigen Aussagen bezüglich des Prozesses macht, bleibt die Frage offen, warum superkongruente Exemplare einen stärkeren Einfluss ausüben. Die Studien 5 und 6 liefern hier jedoch erste Hinweise.

Um den Prozess genauer zu beleuchten, wurde in Studie 5 und 6 untersucht, unter welchen Bedingungen keine Anpassung des Stereotyps in Richtung superkongruenter Exemplare stattfindet. Es wurde angenommen, dass eine superkongruente Abweichung eher als eine quantitative Abweichung aufzufassen ist. Prototyp und Exemplar weisen beide dasselbe stereotype Attribut auf, sie unterscheiden sich nur im Ausmaß, mit dem das Attribut auftritt. Inkongruente Exemplare besitzen dagegen das stereotype Attribut häufig gar nicht. Die Abweichung kann daher als qualitativer Unterschied aufgefasst werden. Vermutlich wird ein qualitativer Unterschied leichter als Abweichung erkannt und führt somit zu der Wahrnehmung einer geringeren psychologischen Nähe zwischen den beiden Stimuli als ein quantitativer Unterschied. Basierend auf dem Selective Accessibility Model (Strack \& Mussweiler, 2000) sollte es im ersten Fall eher zu einem Unähnlichkeitstesten kommen, und als Folge zu einem Kontrasteffekt, während man im zweiten Fall eher Ähnlichkeitstesten und damit Assimilation erwarten kann. Trifft diese Annahme zu, so müsste jede Manipulation, die den Fokus der Aufmerksamkeit auf die Abweichung der superkongruenten Exemplare lenkt, eine Generalisierung verhindern können. Tatsächlich trat in Studie 5 und 6 keine Anpassung des Stereotyps an das superkongruente Exemplar auf, wenn die Teilnehmenden zuvor die Abweichung der Exemplare von der Mehrheit der Kategorie einschätzen sollten.

Da Stereotype häufig mit negativen Konsequenzen, wie Diskriminierung, verbunden sind, ist es ein beunruhigendes Ergebnis, dass Stereotype sich leicht polarisieren lassen. Mit Studie 7 ist die Frage verbunden, ob es einfache Mechanismen gibt, die zur 
einer Verhinderung der Generalisierung beitragen können. Aus der bisherigen Forschung zur Veränderung von Stereotypen weiß man, dass zusätzliche, eigentlich neutrale Information über ein inkongruentes Exemplar, häufig dazu führt, dass das Stereotyp nicht an das Exemplar angepasst wird. Studie 7 zeiget einen ähnlichen Befund für superkongruente Exemplare, auch wenn andere Mechanismen zugrunde liegen mögen.

Zusammenfassend liefern die vorliegenden Studien einen Beitrag zum Verständnis von Stereotypen. Bisherige Befunde zum Einfluss von inkongruenten Exemplaren auf Stereotype konnten um den Einfluss von superkongruenten Exemplaren erweitert werden. In Übereinstimmung mit früheren Studien war der Einfluss von inkongruenten Exemplaren in den vorliegenden Studien eher gering, superkongruente Exemplare dagegen führten häufig zu einer Anpassung, und damit Polarisierung des Stereotyps. Allerdings scheint dieser Prozess nicht unaufhaltsam zu sein. Die vorliegenden Studien liefern gleichzeitig erste Anhaltspunkte wie einer Polarisierung von Stereotypen entgegengewirkt werden kann. 


\section{CURRICULUM VITAE}

\section{Persönliche Angaben}

Name:

Geburtsdatum:

Geburtsort:

Familienstand:

\section{Werdegang}

$1984-1988$

$1988-1997$

1997

$1997-2003$

Sept. 2003

Okt. 2004- Nov 2004

Dez. 2004 - März 2005

April 2004 - Sept. 2004

Ab Oktober 2004
Mirjam Katharina Dolderer

07.04 .1978

Schwäbisch Gmünd, Baden-Württemberg

ledig
Uhland-Grundschule, Schwäbisch Gmünd

Scheffold-Gymnasium, Schwäbisch Gmünd

Abitur

Universität Mannheim, Diplomstudiengang Psychologie

Diplom in Psychologie

Praktikum Filmfestival Mannheim-Heidelberg

Wissenschaftliche Mitarbeiterin am Lehrstuhl für Mikrosoziologie und Sozialpsychologie, Universität Mannheim

Praktikumsinitiative „Creative Village”, Berlin

Promotions-Stipendiatin des Internationalen GraduiertenKollegs „Konflikt und Kooperation zwischen sozialen Gruppen"“ 


\section{EHRENWÖRTLICHE ERKLÄRUNG}

Hiermit erkläre ich, dass mir die geltende Promotionsordnung der Fakultät für Sozial- und Verhaltenswissenschaften der Friedrich-Schiller-Universität Jena bekannt ist.

Ferner erkläre ich, dass ich die vorliegende Arbeit selbst und ohne unzulässige Hilfe Dritter angefertigt habe. Alle von mir benutzten Hilfsmittel, persönliche Mitteilungen und Quellen sind in der Arbeit angegeben. Insbesondere habe ich hierfür nicht die Hilfe eines Promotionsberaters in Anspruch genommen und Dritte haben weder unmittelbar noch mittelbar geldwerte Leistungen von mir für Arbeiten erhalten, die im Zusammenhang mit dem Inhalt der vorgelegten Dissertation stehen.

Die Arbeit wurde weder im In- noch Ausland in gleicher oder ähnlicher Form einer anderen Prüfungsbehörde vorgelegt. Weder früher noch gegenwärtig habe ich an einer anderen Hochschule eine Dissertation eingereicht.

Ich versichere, dass ich nach bestem Wissen die reine Wahrheit gesagt und nichts verschwiegen habe. 